\title{
AN ATLAS OF H II REGIONS IN NEARBY SEYFERT GALAXIES
}

\author{
I. N. Evans, ${ }^{1,2}$ A. P. KoratKar, ${ }^{1}$ T. StorChi-Bergmann, ${ }^{3}$ H. KirkPatrick, ${ }^{4}$ \\ T. M. HeCKMAN, ${ }^{1,5}$ AND A. S. WILSON ${ }^{1,4}$ \\ Received 1995 August 16; accepted 1995 December 5
}

\begin{abstract}
Continuum-subtracted $\mathrm{H} \alpha$ images of 17 nearby Seyfert galaxies are presented. The images are calibrated astrometrically using the Hubble Space Telescope Guide Star Catalog reference frame. For each galaxy, an inventory of $\mathrm{H}$ II regions detected using the COSMOS image classifier and centroider is provided. Salient features of each galaxy are described, including the distribution of the $\mathrm{H}$ II regions with reference to the galaxy morphology. Relevant abundance data from the literature are noted for each object. These images will find use as finding charts for follow-up spectrophotometric investigations of abundances, abundance gradients, and kinematics of $\mathrm{H}$ II regions in active galaxies, and for detailed studies of $\mathrm{H}$ II region populations in these objects.
\end{abstract}

Subject headings: atlases - galaxies: Seyfert - H II regions

\section{INTRODUCTION}

Detailed photoionization models for active galactic nuclei (AGNs) have been developed over the past two decades, yet the elemental abundances of the near-nuclear gas have not been thoroughly investigated. Since the cooling efficiency of the interstellar gas, and therefore the predicted emission-line spectrum, is strongly dependent on the relative abundances of the elements heavier than helium (in particular oxygen), an accurate knowledge of the elemental abundances present in the near nuclear gas is critical for the precise application of these models. Virtually all extant AGN photoionization models assume that the elemental abundances are a priori solar, or very nearly so. Other models have attempted to estimate the relative abundances of the principal elements by assuming a given form for the ionizing spectrum and adjusting the predicted emission-line strengths to match the observations by altering the elemental abundances. For example, using this technique, Storchi-Bergmann \& Pastoriza (1990) have concluded that the nitrogen abundance of the narrow-line region of a sample of 177 low-ionization nuclear emissionline regions (LINERs) and Seyfert 2 galaxies can reach up to 5 times the solar value. This technique has gained considerable popularity over the last 5 years, since the Hubble Space Telescope has provided the capability to obtain spatially resolved spectrophotometry of the narrow line region in nearby AGNs. Although these data provide significant new and valuable constraints for the models of the ionized material in the active nucleus, the technique involves a circular argument. Since the form of the input ionizing spectrum is observationally very poorly determined at best, the abundance estimated using this method have very large uncertainties.

\footnotetext{
${ }^{1}$ Space Telescope Science Institute, 3700 San Martin Drive, Baltimore, MD 21218.

2 Present address: Smithsonian Astrophysical Observatory, 60 Garden Street, MS-27, Cambridge, MA 02138.

${ }^{3}$ Departamento de Astronomia, IF-UFRGS, CP 15051, CEP 91501970, Porto Alegre, RS, Brazil.

${ }^{4}$ University of Maryland, Department of Astronomy, College Park, MD 20742.

${ }^{5}$ The Johns Hopkins University, Department of Physics \& Astronomy, Homewood Campus, Baltimore, MD 21218.
}

Rather than assuming solar ratio elemental abundances, a much better alternative is to determine them observationally from studies of objects whose physics is relatively well understood. Giant extragalactic $\mathrm{H}$ II regions located within the active galaxy form an excellent choice for this analysis.

The physics of $\mathrm{H}$ II regions forms a simple application of Strömgren's photoionization theory. For the simplest case of a pure hydrogen Strömgren sphere, a direct analytic solution for the ionization equilibrium can be computed. In practical cases in which the relative abundances of the elements other than hydrogen are nonzero, numerical solutions to the ionization balance equations are required. The number of parameters in these models is small, and they are well constrained observationally. Since the exciting photon spectrum is dominated by the hottest and most luminous members of the ionizing OB association (Shields \& Searle 1978), only the elemental abundances and a dimensionless "ionization parameter" are free parameters. Furthermore, empirical studies of giant extragalactic $\mathrm{H}$ II regions have shown that the ionization parameter and the elementaveraged metallicity are correlated statistically (Dopita \& Evans 1986). Therefore, one can determine statistically the abundances of the principal elements responsible for cooling the gas directly solely from the measured relative intensities of the strongest emission lines found in the $\mathrm{H}$ II region spectra.

Because of this simplicity, giant extragalactic $\mathrm{H}$ II regions have been used extensively as observational probes of the properties and physical conditions (for example, elemental abundances, electron density, ionization temperature) of the interstellar medium (ISM) in normal galaxies that have little or no nuclear activity (e.g., Dinerstein 1990, and references cited therein). However, detailed studies of giant $\mathrm{H}$ II regions in galaxies with active nuclei are limited in number (e.g., Pagel et al. 1979; Hawley \& Phillips 1980; Evans \& Dopita 1987; Zaritsky, Kennicutt, \& Huchra 1994; StorchiBergmann, Wilson, \& Baldwin 1995; Storchi-Bergmann, Ardila, \& Wilson 1996).

Observations of giant extragalactic $\mathrm{H}$ II regions in normal galaxies have shown that, statistically, early-type galaxies have shallow abundance gradients and high overall abundances, whereas late-type spirals typically have steep abundance gradients with low abundances near their Holm- 
berg radii (e.g., Shields \& Searle 1978; Pagel, Edmunds, \& Smith 1980; Edmunds \& Pagel 1984; Evans 1986; Roy \& Walsh 1987, 1988; Walsh \& Roy 1989). With the exception of irregular galaxy morphologies for which often no clear trends are visible, elemental abundances tend to increase toward the nucleus irrespective of the Hubble type of the galaxy. Radial inflows of material are required in many chemical evolution models to explain the observed abundance gradients in normal spiral galaxies (e.g., Tinsley \& Larson 1978; Mayor \& Vigroux 1981; Lacey \& Fall 1985).

In contrast to the extensive studies revealing a wide range of elemental abundances and abundance gradients in normal galaxies, the situation in active galaxies is at present poorly understood. Early observations of $\mathrm{H}$ II regions in Seyfert galaxies suggest that the elemental abundances are typically solar or higher, and that the abundance gradients are shallow (Pagel et al. 1979; Hawley \& Phillips 1980; Evans \& Dopita 1987). More recent determinations of the chemical abundances of $\mathbf{H}$ II regions in four other active galaxies (Storchi-Bergmann et al. 1995, 1996) indicate substantial radial abundance gradients and nuclear abundances typically twice solar.

A comprehensive study of the chemical abundances in a statistically complete sample of active galaxies is needed to reach more firm conclusions about the overall abundances and abundance gradients in these objects. Once the radial abundance gradients are well determined, the observed gradient can be extrapolated to derive the elemental abundances in the nucleus. This would then provide a powerful observational basis for constraining the elemental abundances used in photoionization models of the active nucleus itself. A caveat to this method is that within the nuclear ionization region, abundances determined from simple $\mathrm{H}$ II region models (assuming no nuclear radiation field) may be artificially low. This is because the central source of nuclear activity may partially ionize the circumnuclear gas, so raising its ionization parameter. If the conditions in the gas were appropriate, the intensities of the lines emitted by the principal coolants would be increased, and this could mimic the emission-line spectrum from an $\mathrm{H}$ il region with lower elemental abundances. Since the signature of this effect is an abundance gradient that apparently decreases inward (Evans \& Dopita 1987), it is easy to identify which H II regions are affected by the nuclear ionizing radiation field and apply an appropiate correction.

If the abundance gradients seen in active galaxies are statistically different from their counterparts in normal galaxies of similar Hubble type, implying that there is an intrinsic relationship with the nuclear activity rather than a simple dependence on morphological type, then observations of the local ISM will provide a very powerful tool to determine just how the nuclear activity affects the chemical evolution of the ISM in the parent galaxy. There are many ways in which nuclear activity may alter the chemical evolution of the galaxy. For example, nuclear winds, shocks, and radio jets may provide substantial kinetic energy input into the local interstellar medium, resulting in enhanced star formation rates and therefore significantly increased chemical enrichment of the gas through enhanced supernova production. Such processes could result in substantial radial mixing of the ISM through the interaction of nuclear outflows (including enhanced supernova winds) with radial inflows, thus unexpectedly minimizing, rather than enhancing, radial abundance gradients. Strong radial flows are pre- dicted by many galaxy evolutionary models and act to efficiently mix the ISM and so eliminate discontinuities in the physical and chemical properties of the interstellar medium as a function of radius. On the other hand, the few observations of disk $\mathrm{H}$ II regions in Seyfert galaxies that do exist show no clear evidence for abnormal $\mathrm{N} / \mathrm{O}$ or $\mathrm{S} / \mathrm{O}$ ratios that would be expected from a supernova formation rate that has been enhanced by nuclear activity (Evans \& Dopita 1987). This suggests that enhancements of supernovae and star formation triggered by nuclear shocks and winds may be energetically insignificant or do not greatly modify the local stellar mass function in the disk over the timescale of the nuclear activity. On smaller scales, StorchiBergmann \& Pastoriza (1990) find evidence for high N/O, and possibly $\mathrm{S} / \mathrm{O}$, ratios in the narrow-line regions of some LINERs and Seyfert 2 galaxies, and Storchi-Bergmann et al. (1995) find $\mathrm{N} / \mathrm{O}$ enhanced above the solar value by a factor of 2-3 in the star-forming rings of NGC 1097 and NGC 1672. A detailed statistical study is required to detect these effects and to differentiate between alternate scenarios.

As a first step toward determining the elemental abundances and abundance gradients in a statistically significant sample of AGNs, in this paper we present continuumsubtracted $H \alpha$ images and inventories of $H$ II regions for 17 nearby active galaxies. The images are calibrated astrometrically using the Hubble Space Telescope Guide Star Catalog (GSC) as the reference frame, and the coordinates of the brightest $\mathrm{H}$ II regions in each galaxy are measured. These data can be used as a starting point for further detailed studies of giant $\mathrm{H}$ II regons in this sample of active galaxies.

The observations reported here comprise a subset of a sample of galaxies selected on the basis of several criteria. Only nearby active galaxies with radial velocities less than $5000 \mathrm{~km} \mathrm{~s}^{-1}$ reported as having Seyfert characteristics by Véron-Cetty \& Véron (1993) are included. To make the atlas a useful starting point for further spectrophotometric work, the sample objects are restricted to have an isophotal diameter as reported by de Vaucouleurs et al. (1991) of no less than 90" and an isophotal ratio smaller than 2.5:1.

\section{OBSERVATIONS AND REDUCTIONS}

All the CCD images included in this atlas were obtained as part of other investigations and include a narrow-band interference filter image centered near $\mathrm{H} \alpha \lambda 6563$ and an associated continuum filter image. Although a narrow filter bandwidth is desirable to isolate pure Balmer emission from the nearby [N II] $\lambda \lambda 6548,6583$ doublet, the range of radial velocities spanned by the sample galaxies and the availability of filters prevented the use of such narrow bandpasses. Therefore, the " $\mathrm{H} \alpha$ " images actually include in addition to the Balmer line flux a component from the $[\mathrm{N}$ II] doublet. No attempt is made to remove this component, since the $[\mathrm{N}$ II] lines are also an emission signature from giant extragalactic $\mathrm{H}$ II regions. As indicated in the observation log (Table 1), the image used to remove the galaxy background continuum was obtained either through an interference filter centered on a predominantly line-free region of the spectrum redward of the $H \alpha \lambda 6563$ emission line, or through a broadband red filter. In the latter case, the line emission from $\mathrm{H} \alpha+[\mathrm{N}$ II] contributes some flux through the broadband filter. However, the flux arising from the galaxy continuum dominates the emission-line 
TABLE 1

ObSERVATION LOG

\begin{tabular}{|c|c|c|c|c|c|c|c|}
\hline Object & Filter & Detector & Source & $\begin{array}{l}\text { Exposure } \\
\text { (s) }\end{array}$ & Date & Classification ${ }^{\mathbf{b}}$ & Morphology \\
\hline NGC $788 \ldots . .$. & $\begin{array}{l}6648 \\
\text { Harris } R\end{array}$ & $\begin{array}{l}\text { TI no. } 2 \\
\text { TI no. } 2\end{array}$ & $\begin{array}{l}\text { KPNO } 2.1 \mathrm{~m} \\
\text { KPNO } 2.1 \mathrm{~m}\end{array}$ & $\begin{array}{l}900 \\
300\end{array}$ & $\begin{array}{l}1989 \text { Jan } 1 \\
1989 \text { Jan } 1\end{array}$ & Seyfert 2 & SA (s) $0 / a$ \\
\hline NGC $1068 \ldots . .$. & $\begin{array}{l}6606 \\
\text { Harris } R\end{array}$ & $\begin{array}{l}\text { TI no. } 2 \\
\text { TI no. } 2\end{array}$ & $\begin{array}{l}\text { KPNO } 2.1 \mathrm{~m} \\
\text { KPNO } 2.1 \mathrm{~m}\end{array}$ & $\begin{array}{r}150 \\
40\end{array}$ & $\begin{array}{l}1989 \text { Jan } 2 \\
1989 \text { Jan } 2\end{array}$ & Seyfert 1 & (RS) SA (rs) b \\
\hline NGC $1097 \ldots . .$. & $\begin{array}{l}6606 / 75 \\
7099 / 80\end{array}$ & $\begin{array}{l}\text { Tek } 1024 \text { no. } 1 \\
\text { Tek } 1024 \text { no. } 1\end{array}$ & $\begin{array}{l}\text { CTIO } 1.5 \mathrm{~m} \\
\text { CTIO } 1.5 \mathrm{~m}\end{array}$ & $\begin{array}{l}600 \\
600\end{array}$ & $\begin{array}{l}1991 \text { Oct } 7 \\
1991 \text { Oct } 7\end{array}$ & Seyfert 1 & SB (s) b \\
\hline NGC $1672 \ldots . .$. & $\begin{array}{l}6606 / 75 \\
7099 / 80\end{array}$ & $\begin{array}{l}\text { Tek } 1024 \text { no. } 1 \\
\text { Tek } 1024 \text { no. } 1\end{array}$ & $\begin{array}{l}\text { CTIO } 1.5 \mathrm{~m} \\
\text { CTIO } 1.5 \mathrm{~m}\end{array}$ & $\begin{array}{l}600 \\
600\end{array}$ & $\begin{array}{l}1991 \text { Oct } 7 \\
1991 \text { Oct } 8\end{array}$ & Seyfert 2 & SB (s) b \\
\hline NGC $2782 \ldots \ldots$ & $\begin{array}{l}6606 \\
\text { Harris } R\end{array}$ & $\begin{array}{l}\text { RCA no. } 2 \\
\text { RCA no. } 2\end{array}$ & $\begin{array}{l}\text { KPNO } 2.1 \mathrm{~m} \\
\text { KPNO } 2.1 \mathrm{~m}\end{array}$ & $\begin{array}{l}900 \\
300\end{array}$ & $\begin{array}{l}1990 \text { Jan } 20 \\
1990 \text { Jan } 20\end{array}$ & Seyfert 2 & SAB (rs) a pec \\
\hline NGC $3081 \ldots \ldots$ & $\begin{array}{l}6606 / 75 \\
7099 / 80\end{array}$ & $\begin{array}{l}\text { Tek } 1024 \text { no. } 1 \\
\text { Tek } 1024 \text { no. } 1\end{array}$ & $\begin{array}{l}\text { CTIO } 1.5 \mathrm{~m} \\
\text { CTIO } 1.5 \mathrm{~m}\end{array}$ & $\begin{array}{l}600 \\
600\end{array}$ & $\begin{array}{l}1992 \text { Apr } 9 \\
1992 \text { Apr } 9\end{array}$ & Seyfert 2 & (R) SAB (r) 0/a \\
\hline NGC $4051 \ldots . .$. & $\begin{array}{l}6563 \\
\text { Harris } R\end{array}$ & $\begin{array}{l}\text { TI no. } 2 \\
\text { TI no. } 2\end{array}$ & $\begin{array}{l}\text { KPNO } 2.1 \mathrm{~m} \\
\text { KPNO } 2.1 \mathrm{~m}\end{array}$ & $\begin{array}{r}255 \\
20\end{array}$ & $\begin{array}{l}1989 \operatorname{Jan} 1 \\
1989 \operatorname{Jan} 1\end{array}$ & Seyfert 1 & $\mathrm{SAB}(\mathrm{rs}) \mathrm{bc}$ \\
\hline NGC $4593 . . . .$. & $\begin{array}{l}6606 / 75 \\
\text { Harris } R\end{array}$ & $\begin{array}{l}\text { RCA no. } 5 \\
\text { RCA no. } 5\end{array}$ & $\begin{array}{l}\text { CTIO } 1.5 \mathrm{~m} \\
\text { CTIO } 1.5 \mathrm{~m}\end{array}$ & $\begin{array}{l}450 \\
150\end{array}$ & $\begin{array}{l}1990 \text { Mar } 19 \\
1990 \text { Mar } 19\end{array}$ & Seyfert 1 & (R) SB (rs) b \\
\hline NGC $4639 \ldots . .$. & $\begin{array}{l}6563 \\
\text { Harris } R\end{array}$ & $\begin{array}{l}\text { Tek no. } 2 \\
\text { Tek no. } 2\end{array}$ & $\begin{array}{l}\text { KPNO } 2.1 \mathrm{~m} \\
\text { KPNO } 2.1 \mathrm{~m}\end{array}$ & $\begin{array}{l}900 \\
150\end{array}$ & $\begin{array}{l}1991 \text { Jun } 7 \\
1991 \text { Jun } 7\end{array}$ & Seyfert 1 & $\mathrm{SAB}(\mathrm{rs}) \mathrm{bc}$ \\
\hline NGC $4939 . . . .$. & $\begin{array}{l}6649 / 76 \\
7146 / 80\end{array}$ & $\begin{array}{l}\text { Tek } 2048 \text { no. } 3 \\
\text { Tek } 2048 \text { no. } 3\end{array}$ & $\begin{array}{l}\text { CTIO } 1.5 \mathrm{~m} \\
\text { CTIO } 1.5 \mathrm{~m}\end{array}$ & $\begin{array}{r}600 \\
60\end{array}$ & $\begin{array}{l}1994 \text { Apr } 6 \\
1994 \text { Apr } 6\end{array}$ & Seyfert & $\mathrm{SA}(\mathrm{s}) \mathrm{bc}$ \\
\hline NGC $5033 \ldots . .$. & $\begin{array}{l}6606 \\
\text { Harris } R\end{array}$ & $\begin{array}{l}\text { TI no. } 2 \\
\text { TI no. } 2\end{array}$ & $\begin{array}{l}\text { KPNO } 2.1 \mathrm{~m} \\
\text { KPNO } 2.1 \mathrm{~m}\end{array}$ & $\begin{array}{l}900 \\
100\end{array}$ & $\begin{array}{l}1988 \text { Dec } 30 \\
1988 \text { Dec } 30\end{array}$ & Seyfert 1 & SA (s) c \\
\hline NGC $5427 \ldots . .$. & $\begin{array}{l}6606 / 75 \\
\text { Harris } R\end{array}$ & $\begin{array}{l}\text { RCA no. } 5 \\
\text { RCA no. } 5\end{array}$ & $\begin{array}{l}\text { CTIO } 1.5 \mathrm{~m} \\
\text { CTIO } 1.5 \mathrm{~m}\end{array}$ & $\begin{array}{l}900 \\
300\end{array}$ & $\begin{array}{l}1990 \text { Mar } 21 \\
1990 \text { Mar } 21\end{array}$ & Seyfert 2 & SA (s) c pec \\
\hline NGC $6300 \ldots . .$. & $\begin{array}{l}6586 / 40 \\
7099 / 80\end{array}$ & $\begin{array}{l}\text { Tek } 1024 \text { no. } 1 \\
\text { Tek } 1024 \text { no. } 1\end{array}$ & $\begin{array}{l}\text { CTIO } 1.5 \mathrm{~m} \\
\text { CTIO } 1.5 \mathrm{~m}\end{array}$ & $\begin{array}{l}600 \\
600\end{array}$ & $\begin{array}{l}1992 \text { Apr } 9 \\
1992 \text { Apr } 9\end{array}$ & Seyfert 2 & $S B(r s) b$ \\
\hline NGC $6814 .$. & $\begin{array}{l}6606 / 75 \\
\text { Harris } R\end{array}$ & $\begin{array}{l}\text { TI no. } 2 \\
\text { TI no. } 2\end{array}$ & $\begin{array}{l}\text { CTIO } 1.5 \mathrm{~m} \\
\text { CTIO } 1.5 \mathrm{~m}\end{array}$ & $\begin{array}{l}900 \\
300\end{array}$ & $\begin{array}{l}1988 \text { Oct } 7 \\
1988 \text { Oct } 7\end{array}$ & Seyfert 1 & $\mathrm{SAB}(\mathrm{rs}) \mathrm{bc}$ \\
\hline NGC $7213 \ldots$. & $\begin{array}{l}6606 / 75 \\
7099 / 80\end{array}$ & $\begin{array}{l}\text { Tek } 1024 \text { no. } 1 \\
\text { Tek } 1024 \text { no. } 1\end{array}$ & $\begin{array}{l}\text { CTIO } 1.5 \mathrm{~m} \\
\text { CTIO } 1.5 \mathrm{~m}\end{array}$ & $\begin{array}{l}600 \\
600\end{array}$ & $\begin{array}{l}1991 \text { Oct } 8 \\
1991 \text { Oct } 8\end{array}$ & Seyfert 1 & SA (s) a \\
\hline NGC $7314 \ldots \ldots$ & $\begin{array}{l}6606 / 75 \\
\text { Harris } R\end{array}$ & $\begin{array}{l}\text { TI no. } 2 \\
\text { TI no. } 2\end{array}$ & $\begin{array}{l}\text { CTIO } 1.5 \mathrm{~m} \\
\text { CTIO } 1.5 \mathrm{~m}\end{array}$ & $\begin{array}{l}900 \\
300\end{array}$ & $\begin{array}{l}1988 \text { Oct } 7 \\
1988 \text { Oct } 7\end{array}$ & Seyfert 1 & SAB (rs) bc \\
\hline NGC $7469 \ldots \ldots$ & $\begin{array}{l}6693 / 76 \\
\text { Harris } R\end{array}$ & $\begin{array}{l}\text { TI no. } 2 \\
\text { TI no. } 2\end{array}$ & $\begin{array}{l}\text { CTIO } 1.5 \mathrm{~m} \\
\text { CTIO } 1.5 \mathrm{~m}\end{array}$ & $\begin{array}{r}225 \\
75\end{array}$ & $\begin{array}{l}1988 \text { Oct } 7 \\
1988 \text { Oct } 7\end{array}$ & Seyfert 1 & (R) SAB (rs) a \\
\hline
\end{tabular}

${ }^{a} \mathrm{KPNO} / \mathrm{CTIO}$ designation.

b Seyfert classification from Véron-Cetty \& Véron 1993.

flux, so that the effect is to decrease slightly the contrast between the line and continuum images.

Standard CCD calibrations, including bias level subtraction and detector flat-field corrections, are applied to the images. No photometric calibrations are applied, however, since these are not available for roughly $\frac{2}{3}$ of the images. Following the standard CCD calibrations, the individual frames are calibrated astrometrically to place the images on the GSC reference frame, and then the continuum frames are subtracted from the emission-line frames to produce a pure $\mathrm{H} \alpha\left(+\left[\mathrm{N}_{\mathrm{II}}\right]\right)$ image of the galaxies. These steps are described below.

For each galaxy, an astrometric calibration for the continuum image is computed to place the image on the GSC reference frame. The continuum frame is compared with the GSC plate scans of the same field, and foreground stars common to both the continuum image and the plate scan are identified. Typically, of order 20 stars distributed throughout the continuum frame are visible also on the plate scan. [The continuum frame is used here in preference to the $H \alpha$ frame since the signal-to-noise ratio $(\mathrm{S} / \mathrm{N})$ of the former is generally larger than for the latter. Therefore, a greater number of foreground stars can be identified to determine better the astrometric solution.] The coordinates of the stars identified common to both the continuum image and the GSC plate scans are measured from the latter, and a least-squares polynomial solution relating the $(x, y)$ posi- tions of the stars on the CCD frame to their GSC coordinates is computed. For each CCD frame, a linear polynomial solution incorporating both translation and rotation forms an accurate astrometric calibration for the image.

For most of the continuum images, the root mean square (rms) uncertainties of the derived astrometric solution are $\sim 0$ " 1 in each axis relative to the GSC reference frame. The worst-case solutions have rms uncertainties of order 0.2 in each of $\xi$ and $\eta$. The accuracy of differential position determinations in a given image is limited by these uncertainties. Absolute astrometry is limited by the astrometric errors inherent in the GSC. The magnitude of these errors depends on the choice of reference catalog but is of order 0 ". 5 (Russell et al. 1990).

After an astrometric solution is determined for the continuum image, the $\mathrm{H} \alpha$ image is calibrated astrometrically, employing the continuum image as the reference frame. This is done rather than computing a separate solution using the GSC plate scans as a reference because the accuracy with which the on- and off-band images can be subtracted is determined in part by the accuracy with which they can be aligned with each other. Using the calibrated continuum image as the reference frame allows more features common to both the $H \alpha$ and continuum images to be identified, since the two images have very similar plate scales, whereas the scale of the GSC plate scans is signifi- 
cantly different. The rms uncertainties of the astrometric calibration required to place the $\mathrm{H} \alpha$ image on the same reference frame as the continuum image are typically a few hundredths of an arcsecond in each axis, and therefore they contribute negligibly in quadrature to the total uncertainties discussed above. The astrometric solution for the $\mathrm{H} \alpha$ image is used as the final astrometric calibration for the field.

Once the astrometric calibrations are computed, the continuum image is warped using bilinear interpolation to the same astrometric solution as the $\mathrm{H} \alpha$ image to remove any residual differences between the $\mathrm{H} \alpha$ and continuum astrometric frames. Following warping, the sky mode is determined and removed from each image. Finally, the continuum frame is subtracted from the $\mathrm{H} \alpha$ frame after suitable scaling of the former. The factor by which the continuum frame is scaled is determined by visual inspection of the resulting image and is the maximum value that does not result in areas of negative residual flux in the subtracted image. With the exception of foreground stars (and, for observations obtained using a broadband $R$ filter, continuum knots associated with $\mathrm{H}$ II regions), the galaxy background detected in the continuum images is morphologically smooth on the scale of a few arcseconds. Therefore, no modifications to either of the $\mathrm{H} \alpha$ or continuum frames are performed to correct for differences between the point-spread functions of the images prior to subtraction.

\section{THE ATLAS}

For each of the galaxies, the continuum-subtracted $\mathrm{H} \alpha$ image is shown in Figure 1 (Plates 1-17), with an astrometric grid in the GSC reference frame overlain. The presentation in this figure uses a linear intensity display to highlight the $\mathrm{H}$ II regions and diffuse $\mathrm{H} \alpha$ emission. In most cases, the dynamic range between the faintest visible $\mathrm{H}$ II regions near the sky level and the brightest $\mathrm{H}$ II regions is larger than can be accommodated using a single linear intensity scale. Rather than saturating the display of the brightest $\mathrm{H} \alpha$ sources or reducing the contrast between the faintest objects and the sky background to an unacceptable level, we have chosen in these cases to use an intensity scale that is wrapped as necessary to enhance the detail of the $\mathrm{H}$ II region morphology. We have chosen further not to edit the images to remove cosmetic defects. In general, such features are readily apparent in the images, allowing the reader to distinguish regions in which care is required when interpreting the data. The differing contributions of stellar continuum fluxes through the on- and off-band filters, and small variations between the point-spread functions for the emission-line and continuum images, produce residual image features for any foreground stars that are included in the field of view of the CCD frames. Although these stellar profiles are not correctly removed in the continuumsubtracted emission-line images, we make no attempt to eliminate them manually since $(a)$ the residual stellar features are quite distinctive, and $(b)$ their presence aids the reader in identification and orientation of the field on the sky.

The COSMOS image classifier and centroider (Lutz 1979; MacGillivray \& Stobie 1984) is used to detect and measure positions of sources in the continuum-subtracted $\mathrm{H} \alpha$ image. Table 2 lists for each galaxy all the COSMOS identified inventory objects that are detected at the $95 \%$ confidence level. For each inventory object, the astrometric position of the object center of gravity (equinox J2000), the object area (see below), the integrated and peak pixel intensities above the sky background, the ellipticity $(1-b / a$, where $a$ is the semimajor axis and $b$ is the semiminor axis), and the P.A. of the major axis, are given. Comparison of the integrated intensity and object area provides a simple estimate of the relative mean surface brightness in emission and can be used as a selection criterion for future spectrophotometric studies.

To identify sources spanning a wide range of peak intensities, several runs of the classifier with different object detection thresholds are executed. The object detection thresholds for each continuum-subtracted $\mathrm{H} \alpha$ image are determined by visual inspection. For emission-line sources that are isolated, the lowest detection threshold that defines the source clearly is used to determine the inventory information for that object. However, if an object is located in a crowded region of the frame, an intensity threshold that is high enough to isolate the source is used. Therefore, the inventory information in Table 2 will understate the total area and integrated intensity for sources in crowded regions. This approach for crowded sources is chosen rather than simply summing over the extended emission region because we expect the inventory information to be used to identify sources for future multiobject fiber spectrograph observations; for this purpose, small-scale source intensity information is more important than extended source data.

The ability to identify individual emission-line sources depends on the local $\mathrm{S} / \mathrm{N}$ of the image, the contrast between the source and the surrounding background, and the source morphology and crowding. Isolated compact objects can be detected down to $\mathrm{S} / \mathrm{N} \sim 1.5$, with some incompleteness. However, we restrict the inventory information in Table 2 to objects detected at the $95 \%$ confidence level. The centerof-gravity centroid position determined by the COSMOS classifier for these objects is repeatable to $\sim 0$ ". $1-0$ ". 2 over a wide range of intensity threshold settings. For noncompact objects and crowded regions, the morphology of the source determines how easily individual components can be detected. Faint components located in close proximity to bright components are not well detected by the COSMOS classifier, and extended regions associated with a bright compact source may or may not be included, depending on the choice of intensity thresholds and the rate at which the surface brightness falls off. These biases may affect significantly number counts of objects, but they are not critical for identifying sources for further study. Although positions measured using the COSMOS classifier in general repeat accurately for a specific intensity threshold setting, the lack of symmetry of the local surface brightness morphology for crowded or extended regions results in the center-of-gravity position having a (possibly strong) intensity threshold dependence.

In some cases, isolated compact $\mathrm{H}$ il regions cannot be distinguished easily from faint foreground stars. This is particularly true for galaxies at low galactic latitude, and also for those objects for which the continuum image is obtained using a broadband $R$ filter that includes the $\mathrm{H} \alpha+[\mathrm{N}$ II] emission-line complex. We exclude objects for which the identification as an emission-line source is ambiguous. However, for fields with numerous foreground stars, some of the faintest objects may be erroneously classified as emission-line sources. We estimate that this contamination 


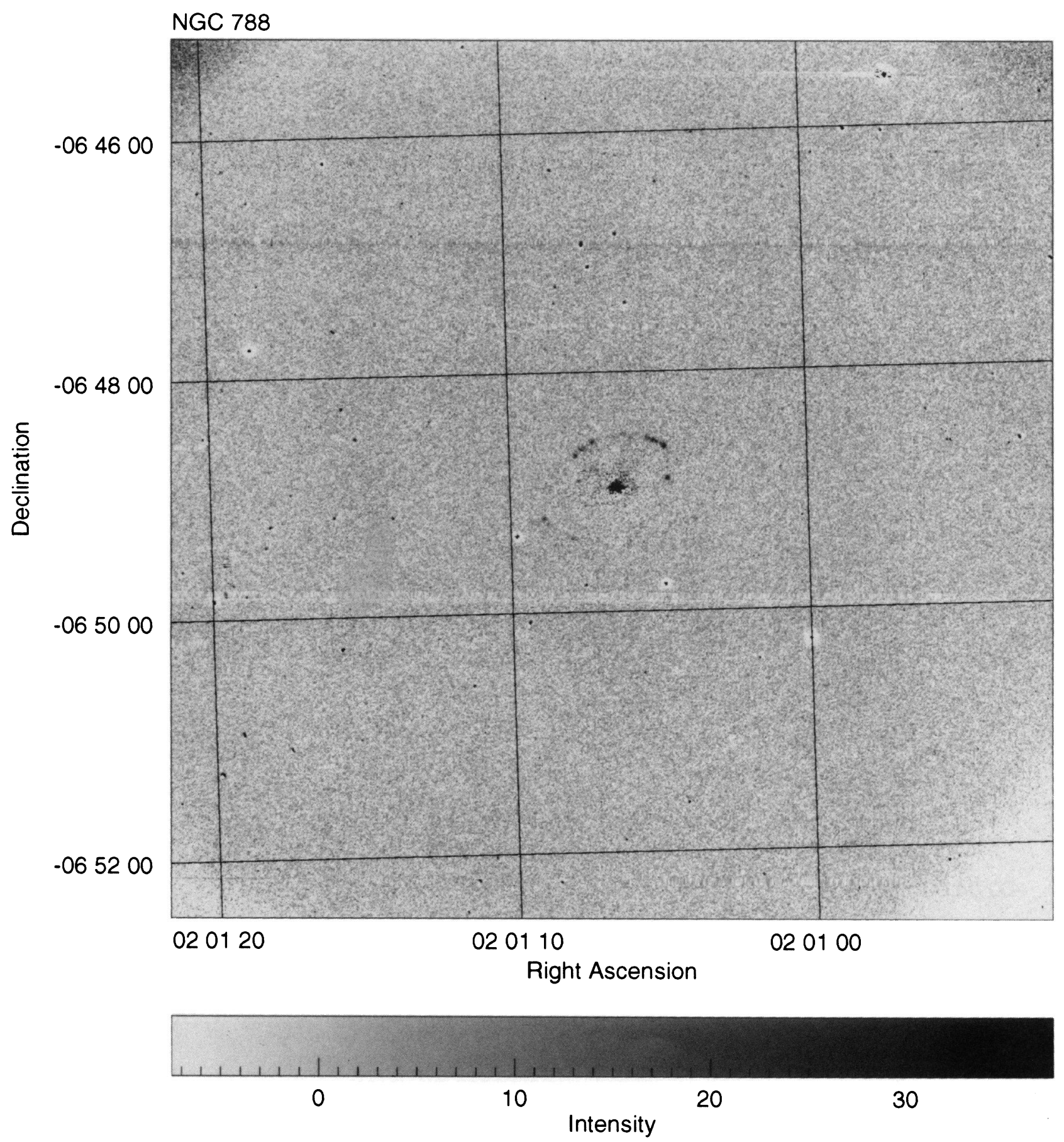

Fig. 1.-Continuum-subtracted $\mathrm{H} \alpha$ images for each of the 17 Seyfert galaxies included in the $\mathrm{H}$ II region atlas. Coordinate grid overlays are in the GSC reference frame (J2000). The data are scaled linearly in DN as shown by the intensity bar below each image.

Evans et al. (see 105, 96) 

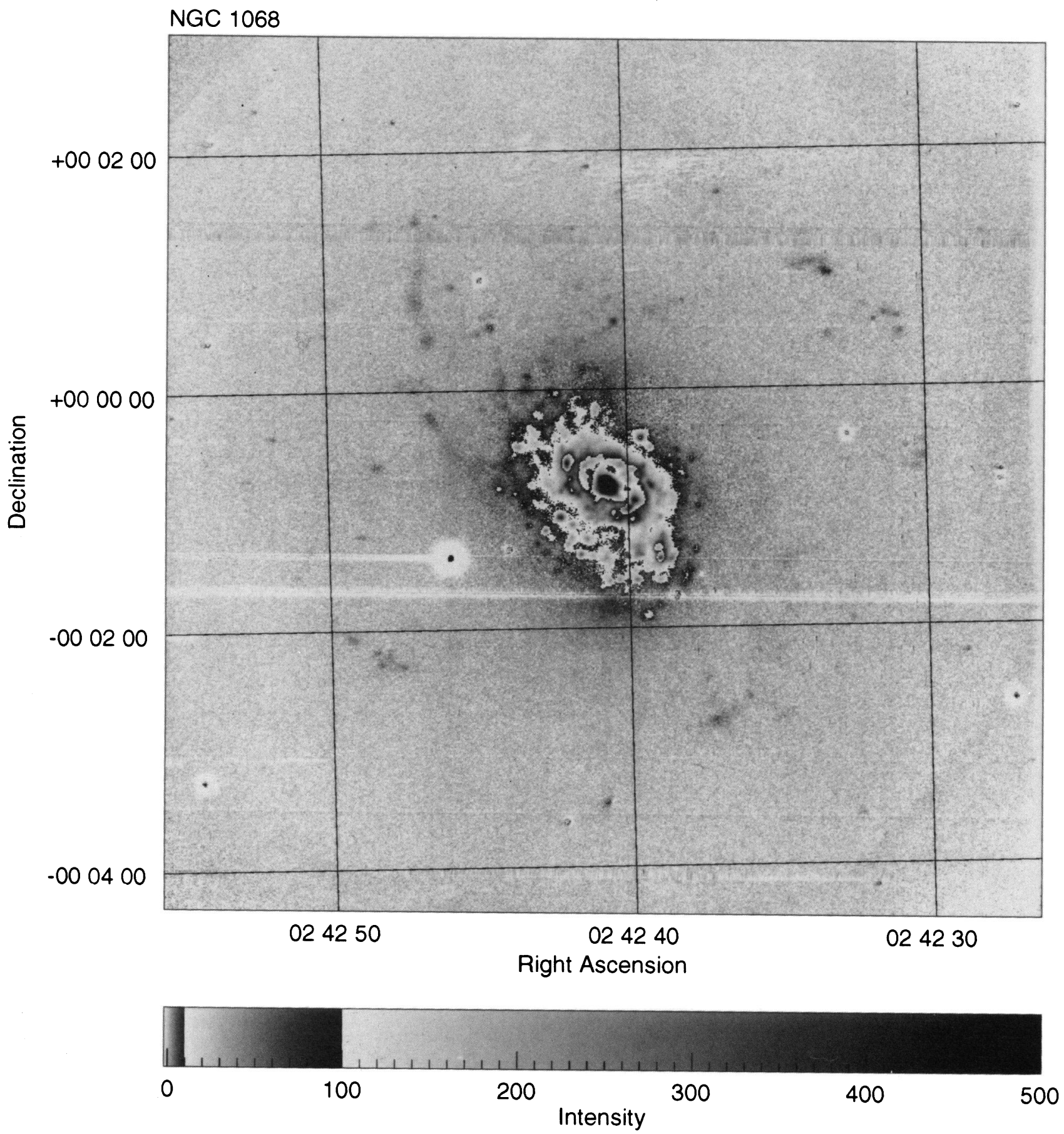

Fig. 1.-Continued

Evans et al. (see 105, 96) 


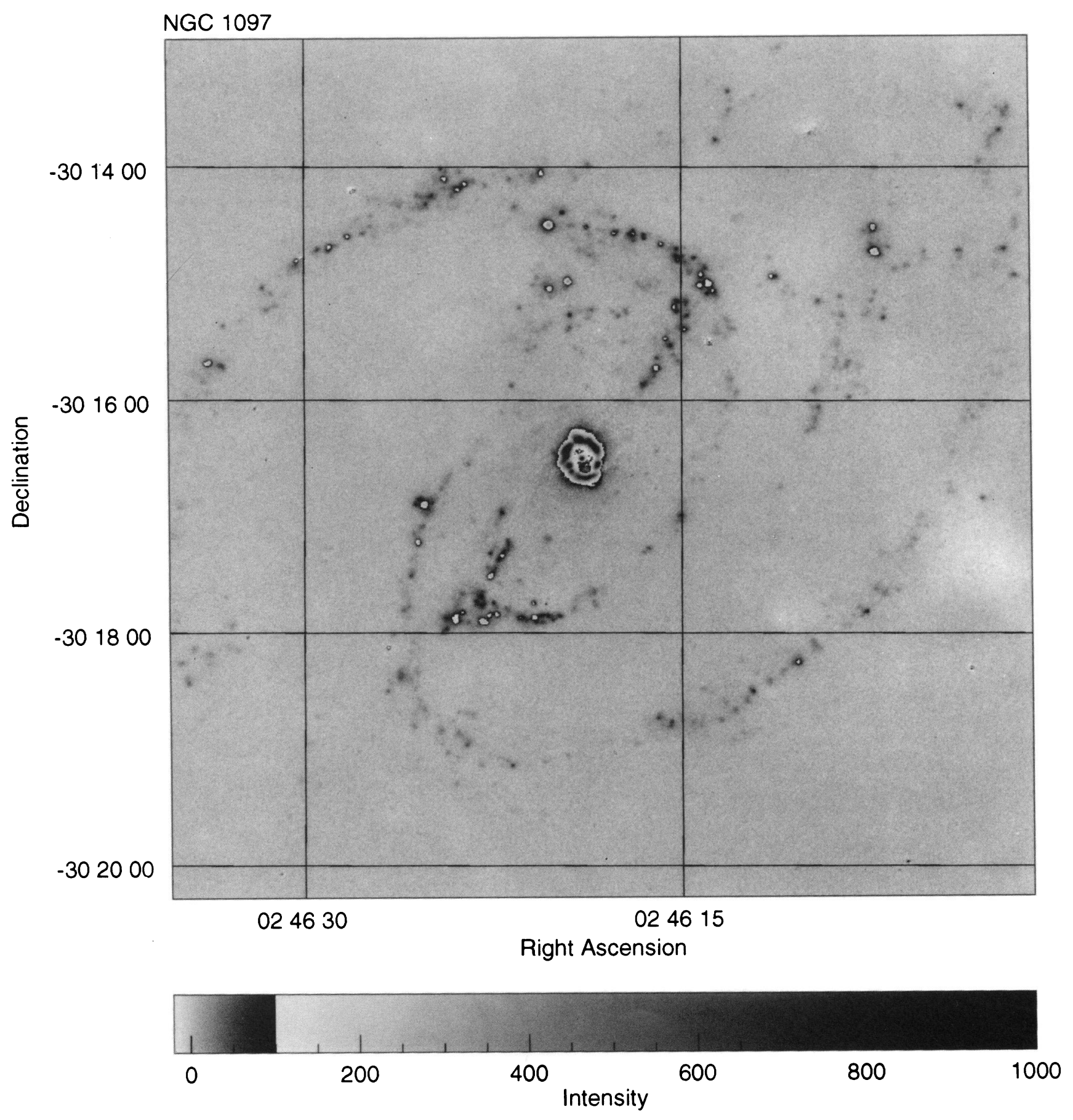

FIG. 1.-Continued

Evans et al. (see 105, 96) 


\section{PLATE 4}

10
1
1
10
10
$1-1$
10
10
10
10
10
10
10
10
$1-1$
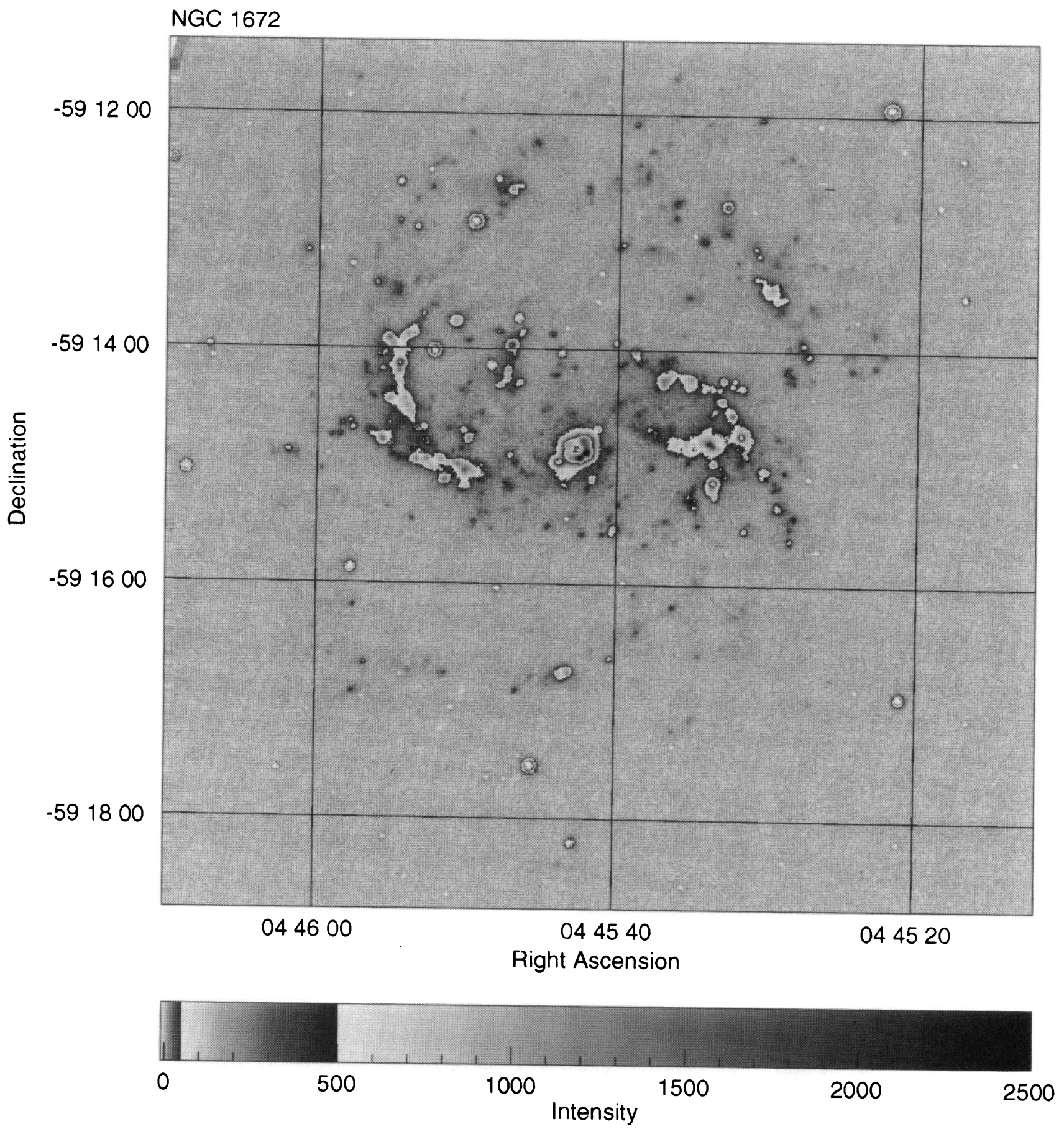

FIG. 1.-Continued

Evans et al. (see 105, 96) 

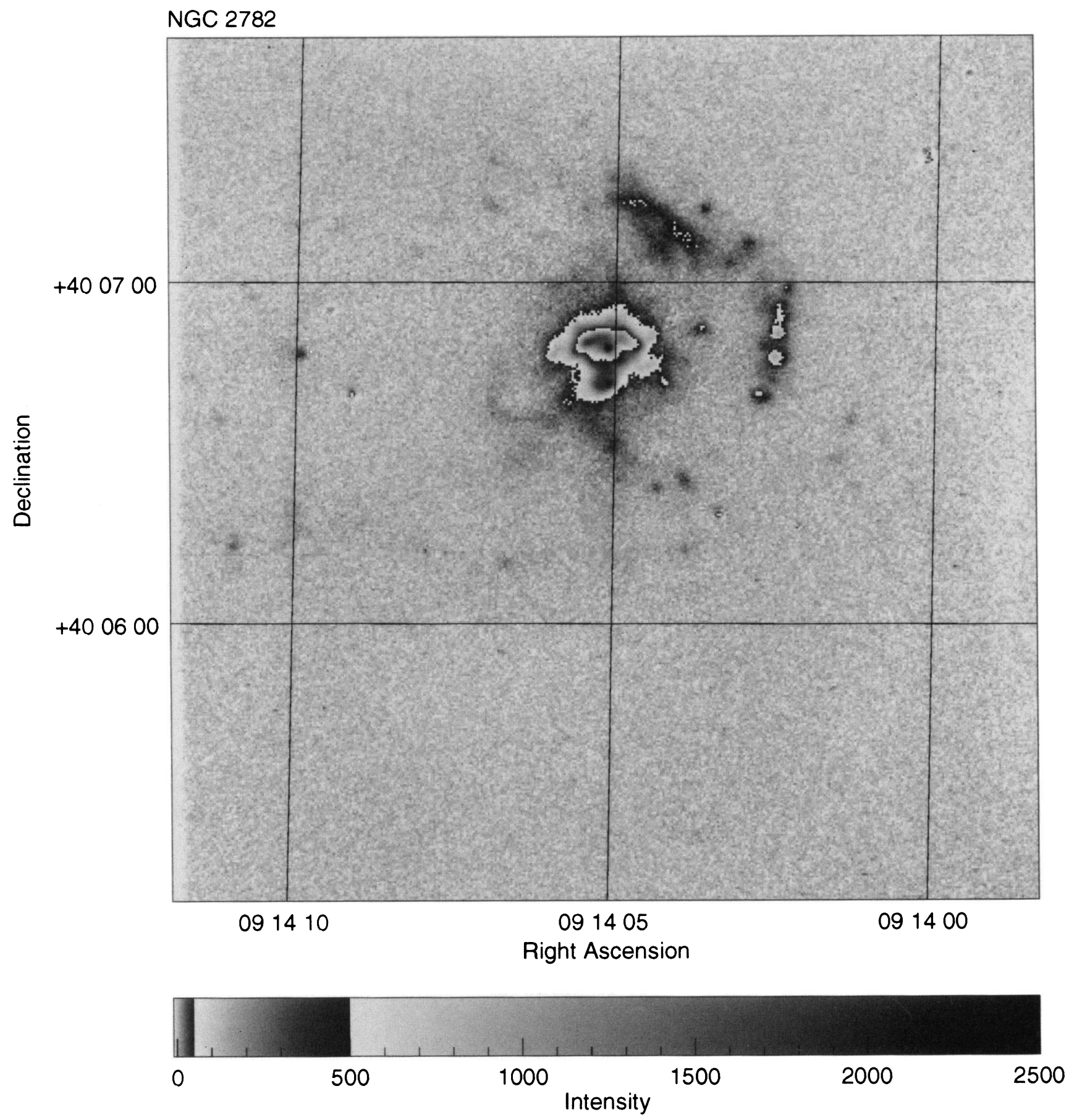

FIG. 1.-Continued

Evans et al. (see 105, 96) 

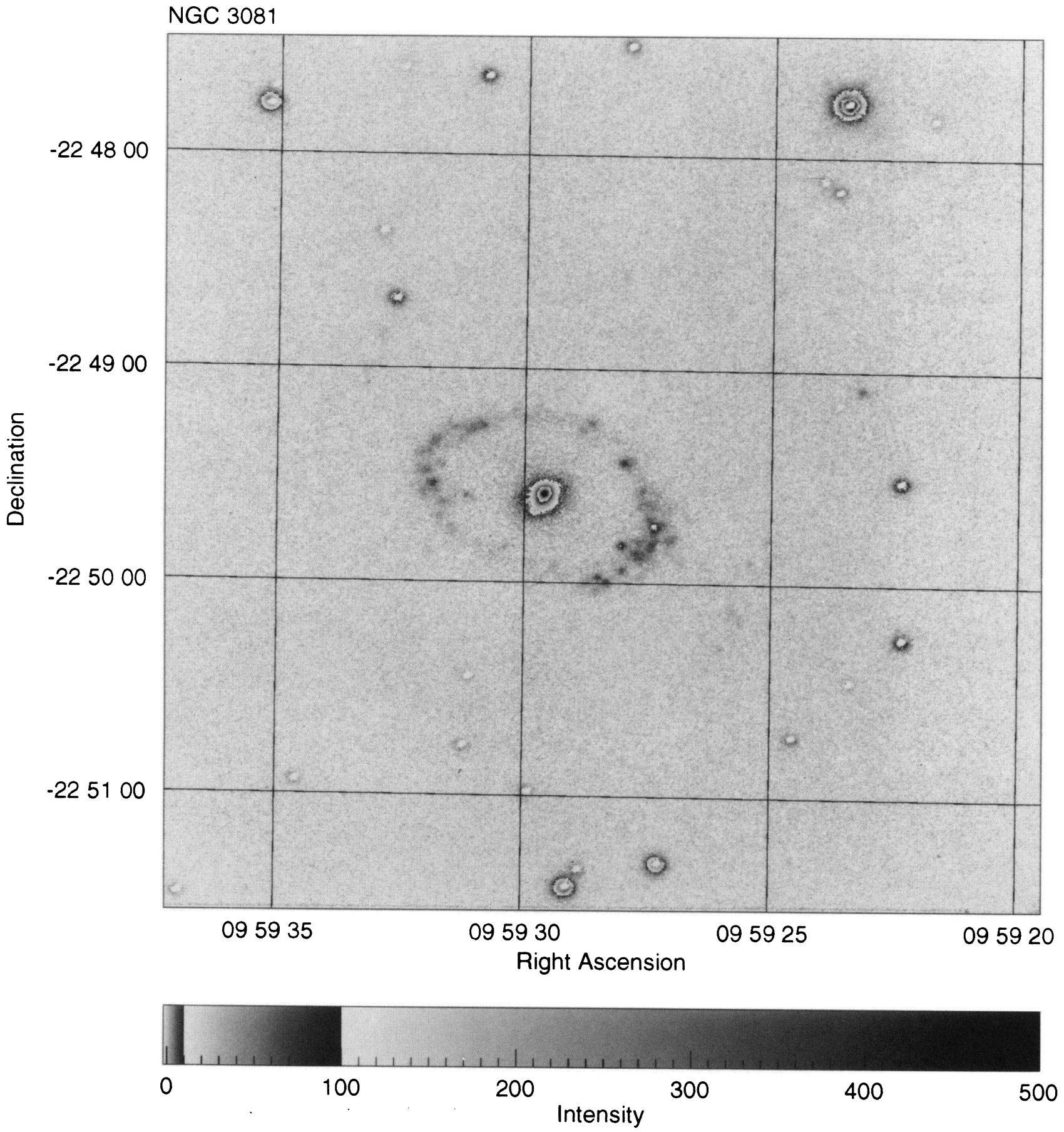

Fig. 1.-Continued

Evans et al. (see 105, 96) 

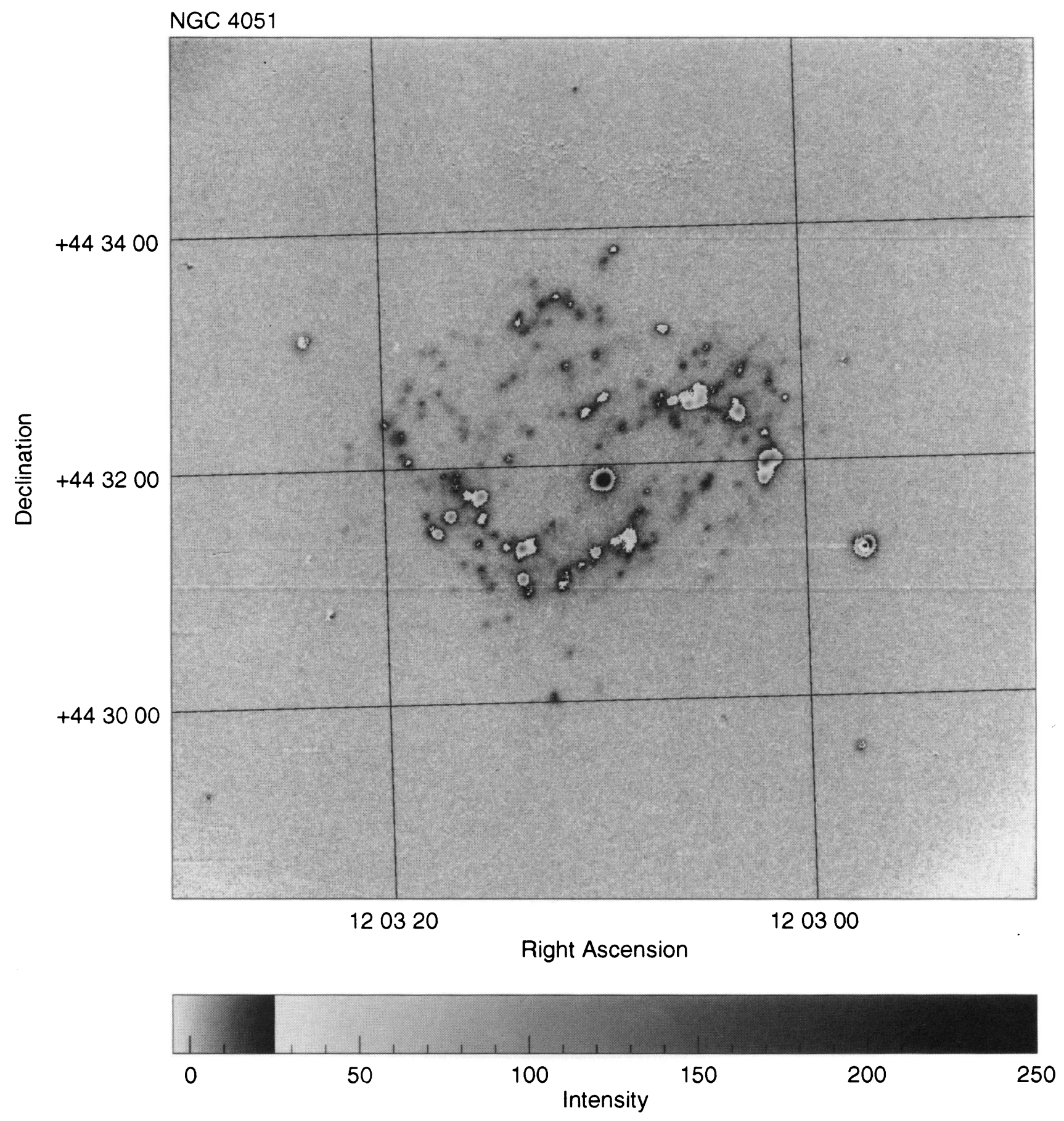

FIG. 1.-Continued

Evans et al. (see 105, 96) 
19.1

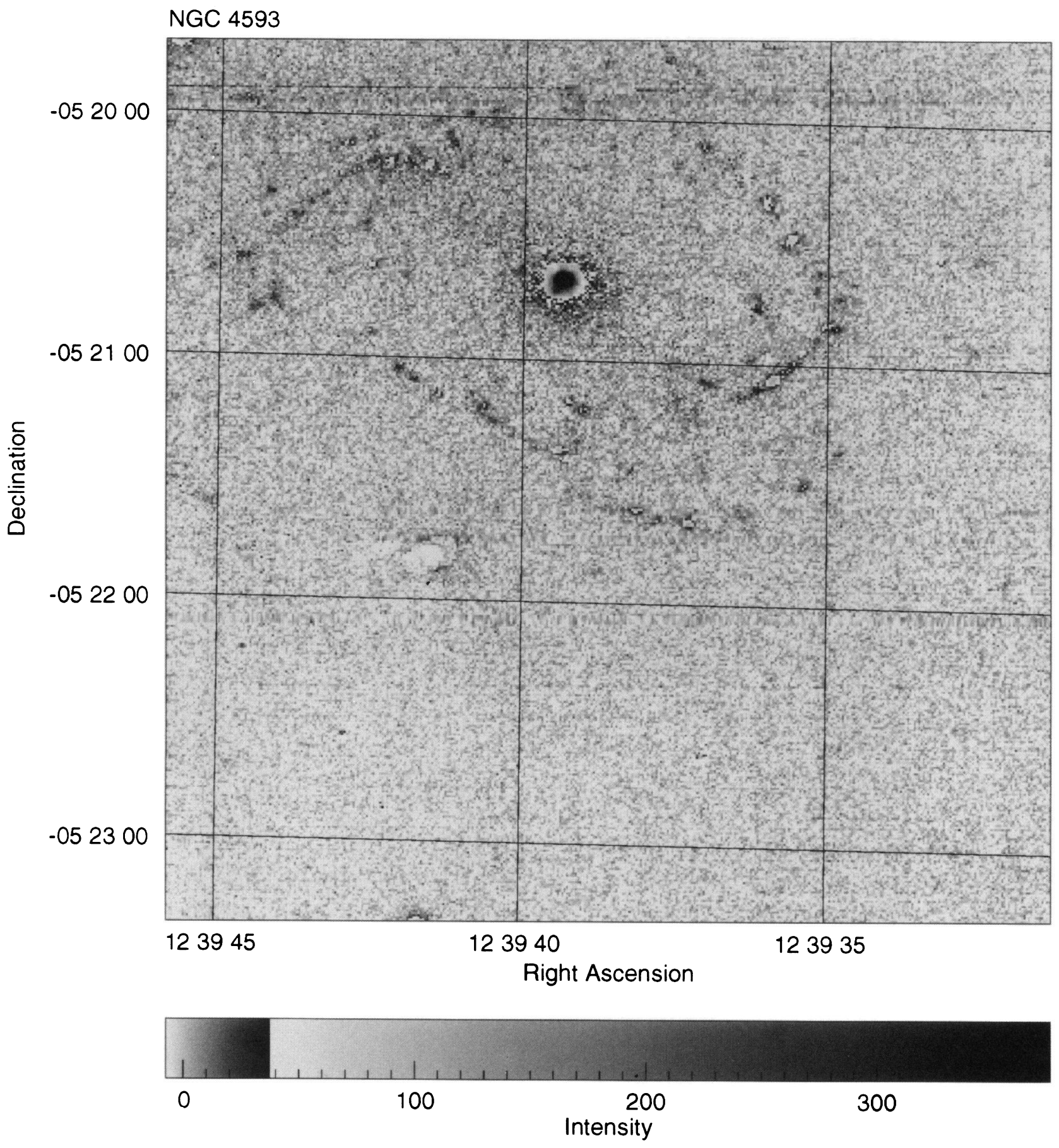

Fig. 1.-Continued

Evans et al. (see 105, 96) 
PLATE 9

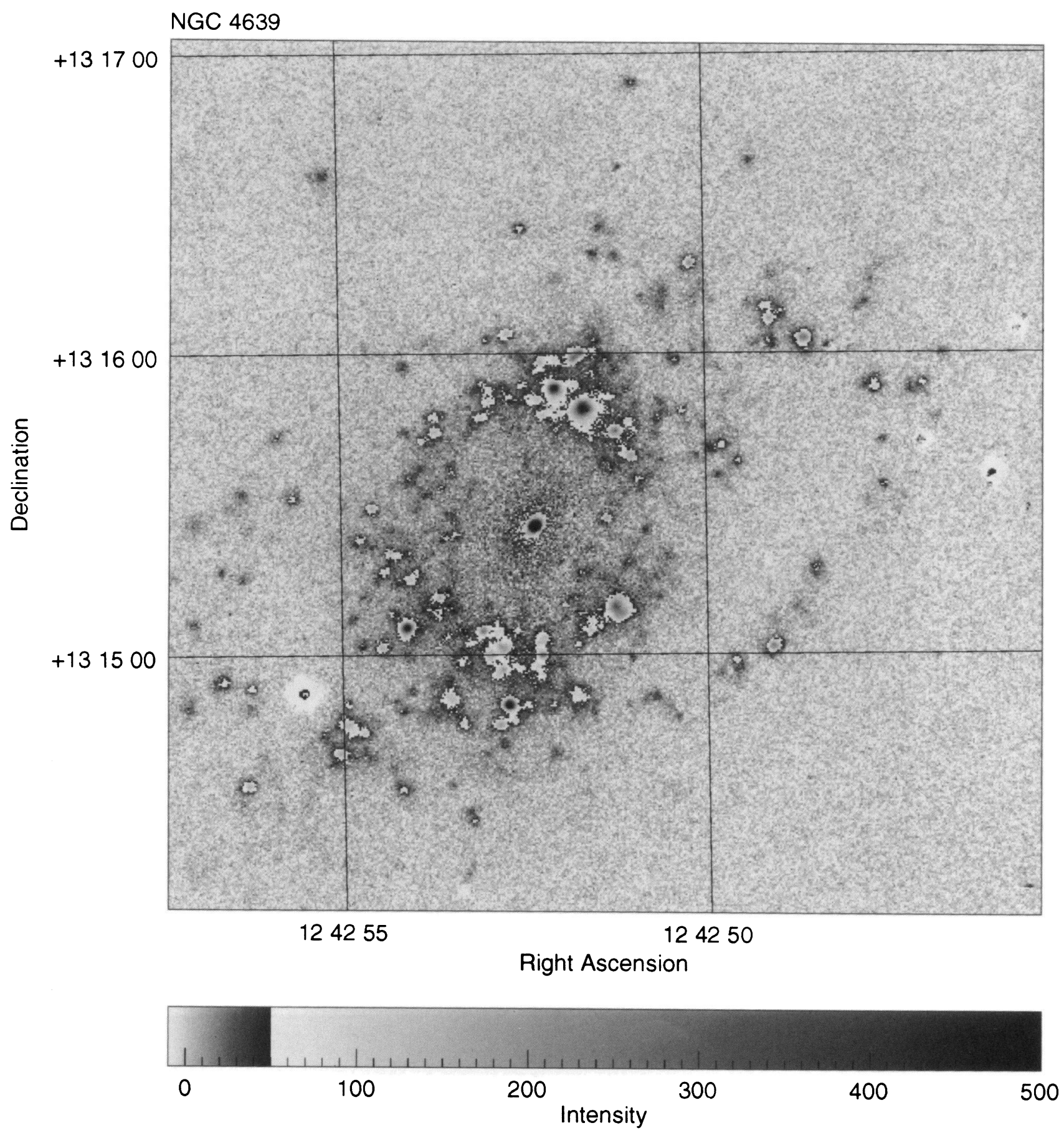

Fig. 1.-Continued

Evans et al. (see 105, 96) 

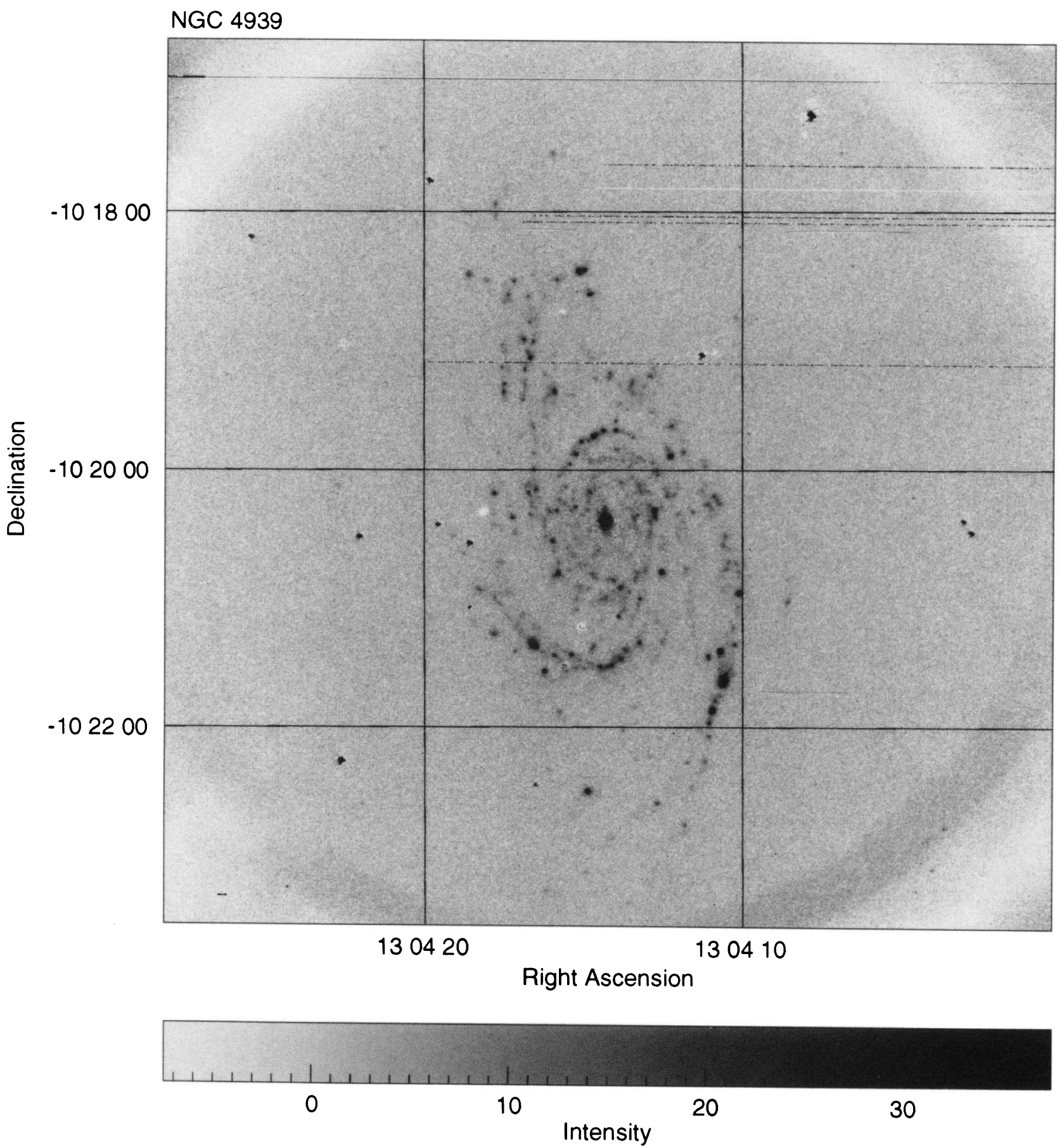

20

30

FIG. 1.-Continued

Evans et al. (see 105, 96) 
PLATE 11

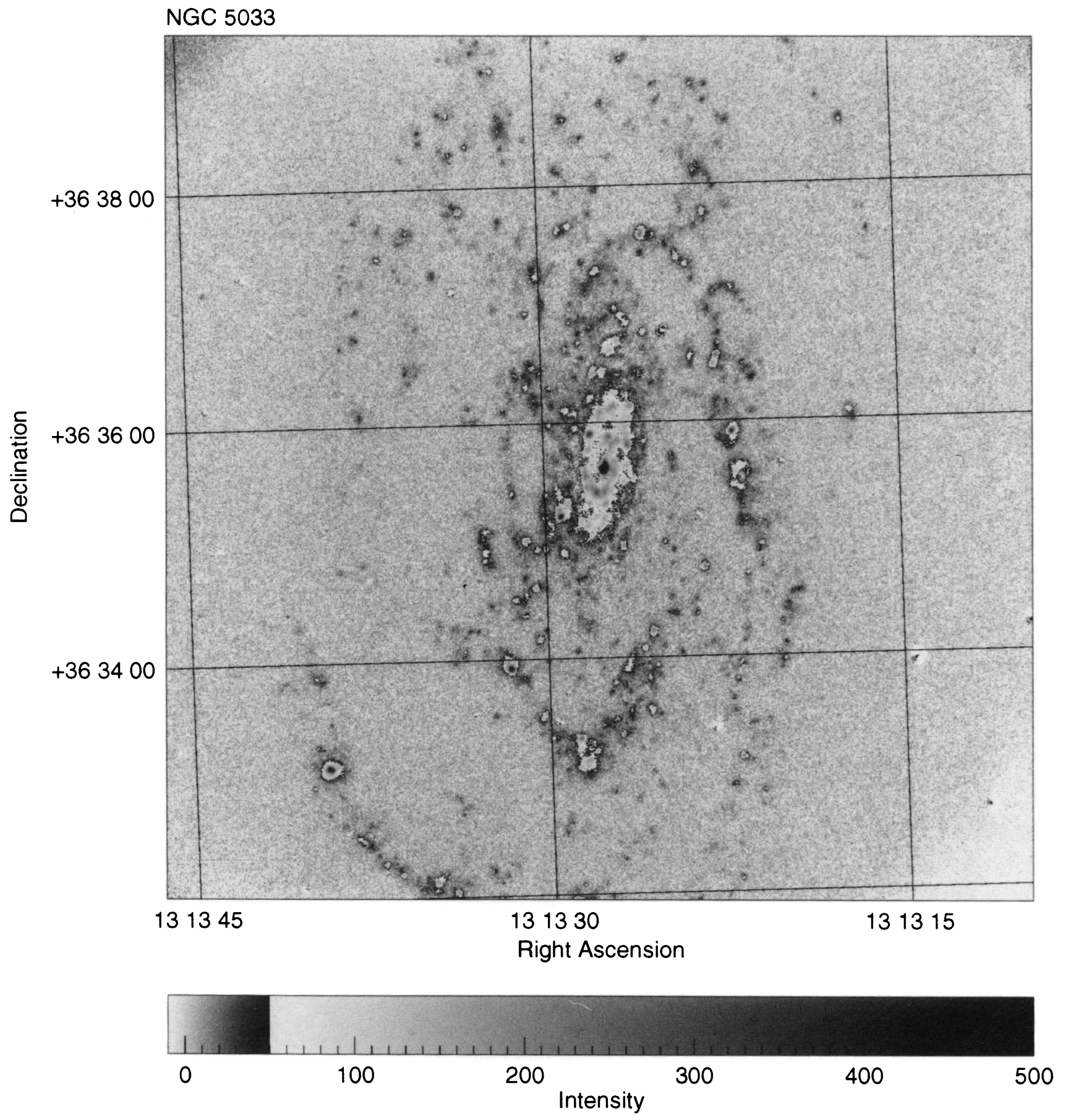

Fig. 1.-Continued

Evans et al. (see 105, 96) 


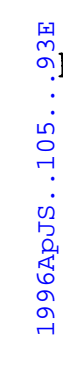
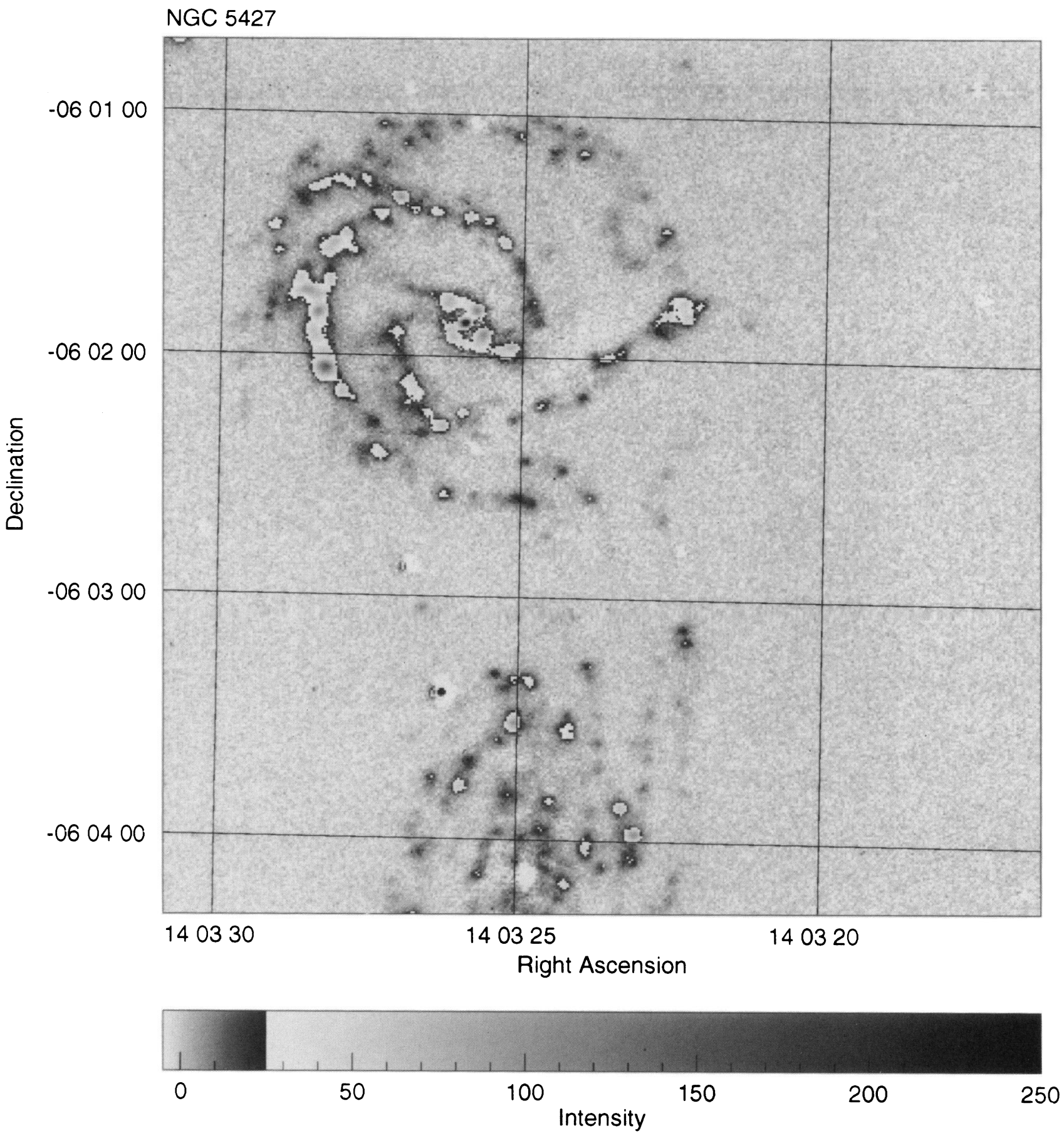

Fig. 1.-Continued

Evans et al. (see 105, 96) 


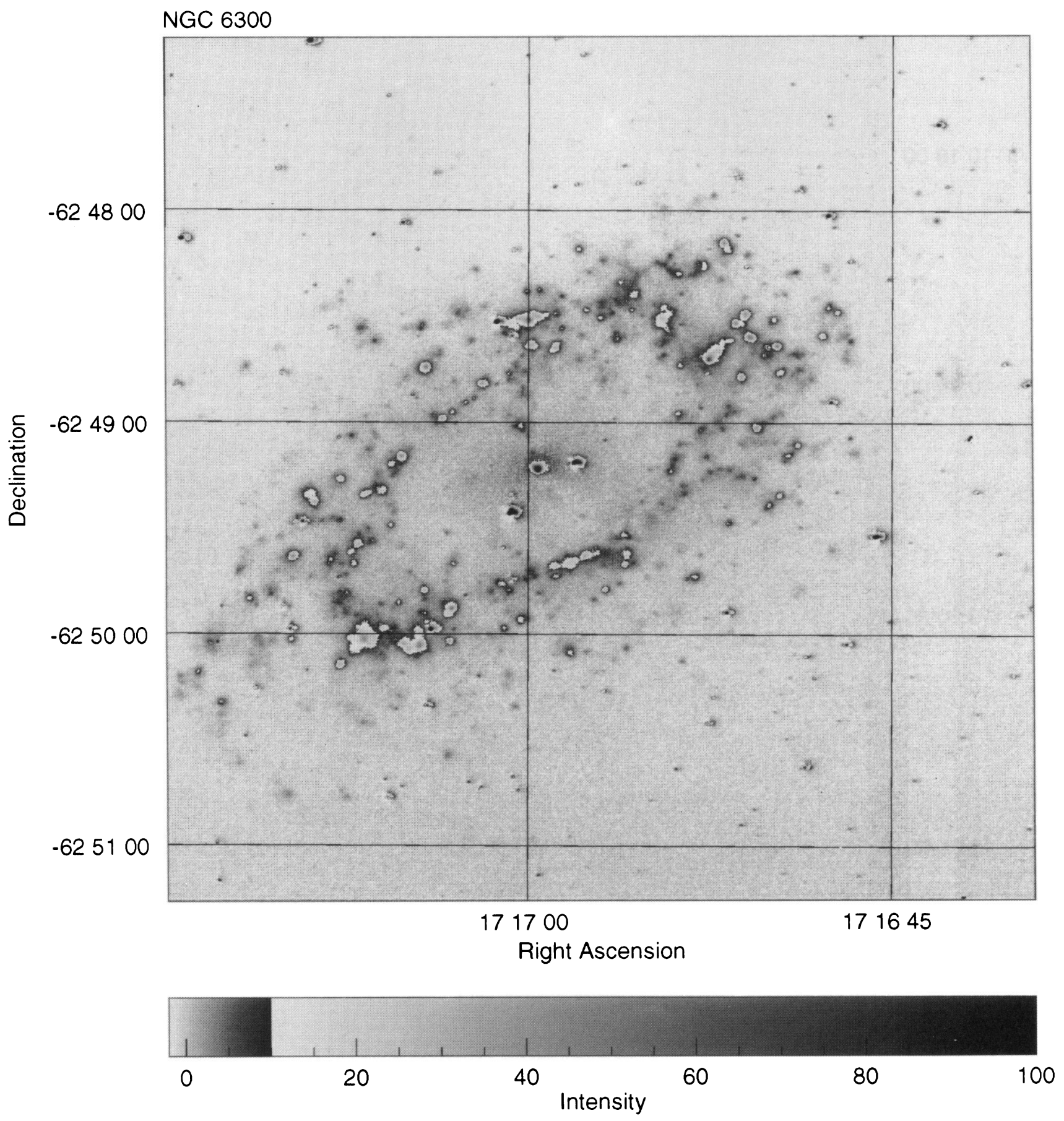

Fig. 1.-Continued

Evans et al. (see 105, 96) 


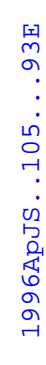

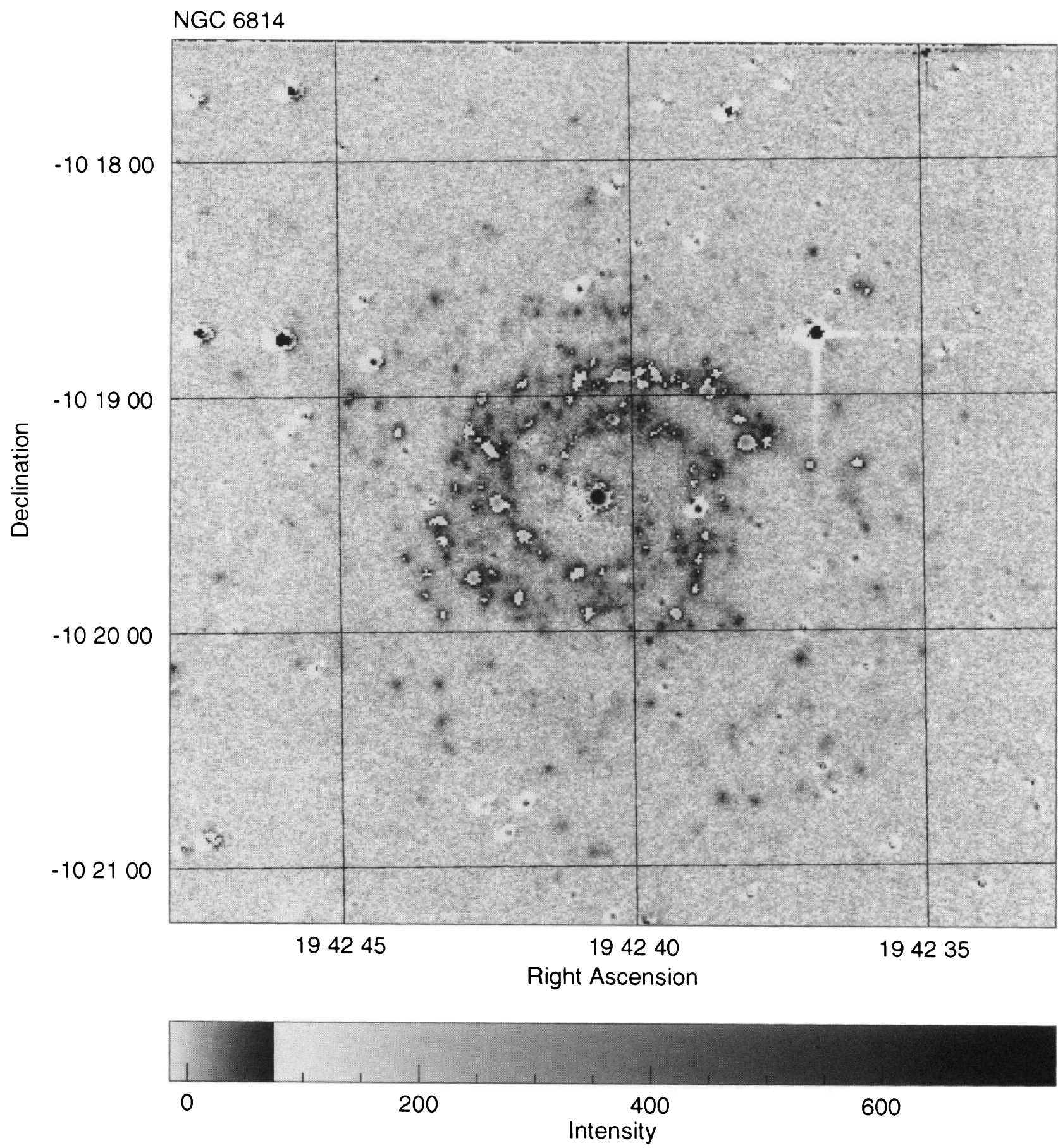

Fig. 1.-Continued

Evans et al. (see 105, 96) 


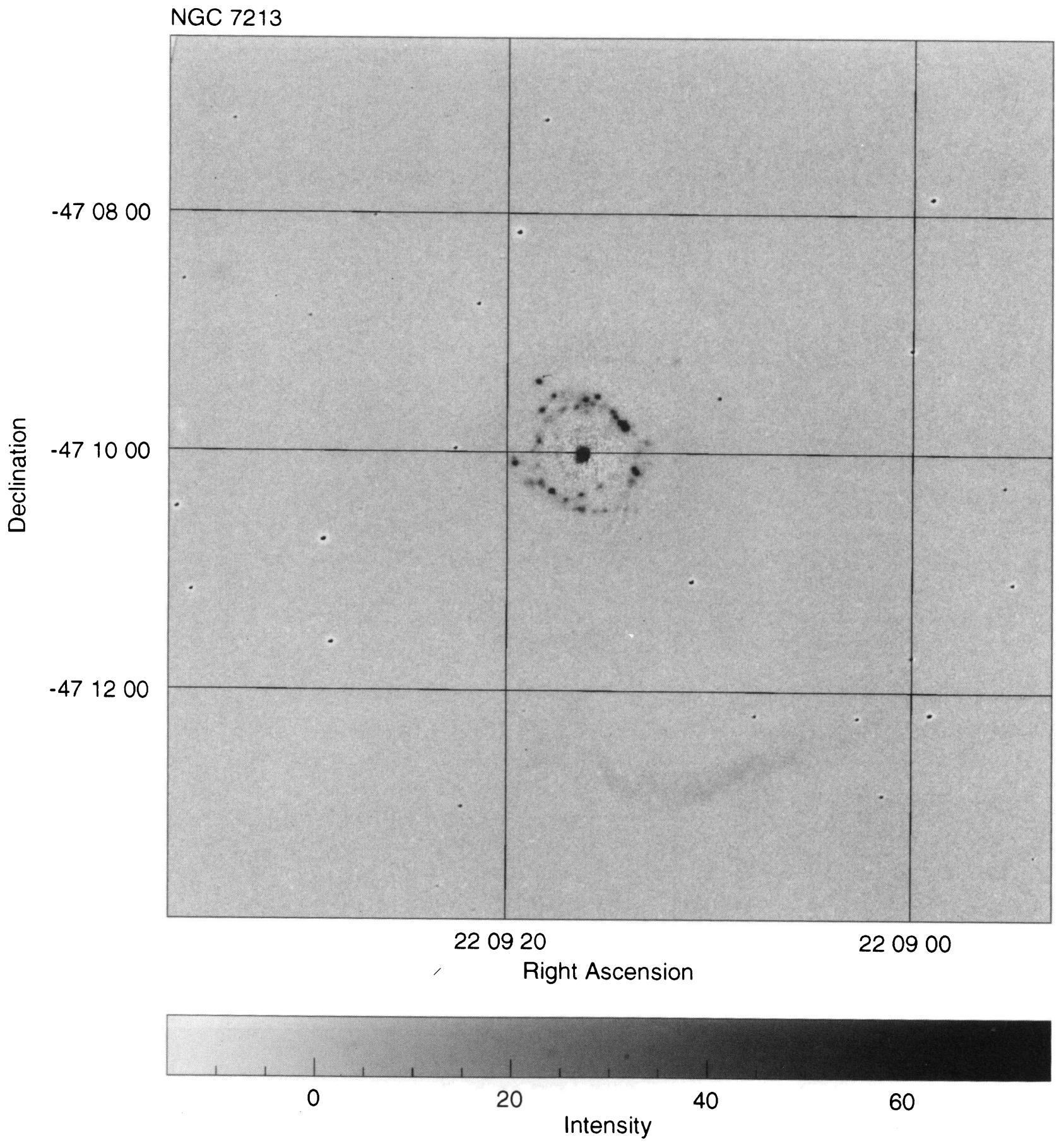

Fig. 1.-Continued

Evans et al. (see 105,96$)$ 


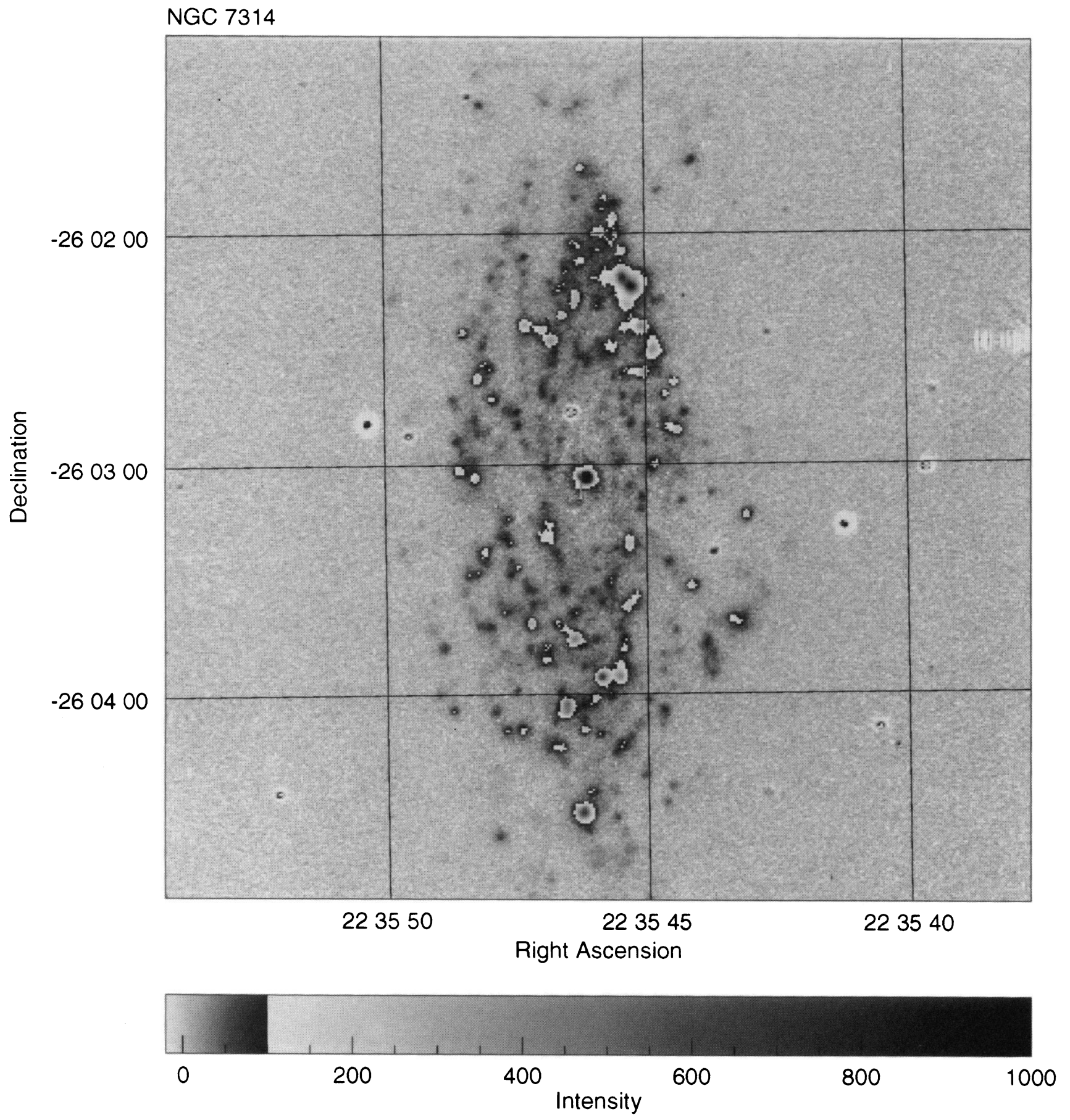

FIG. 1.-Continued

Evans et al. (see 105, 96) 


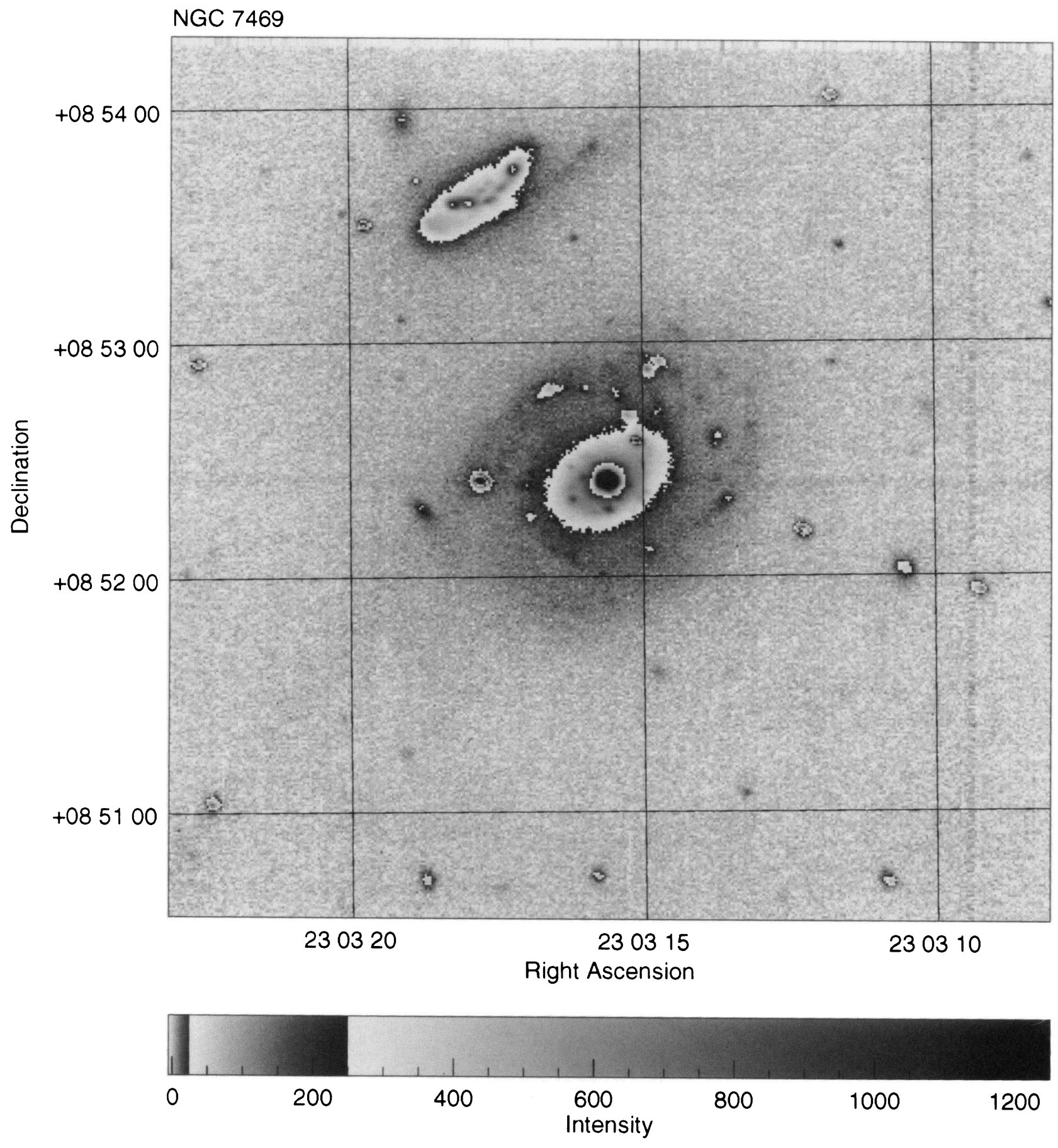

Fig. 1.-Continued

Evans et al. (see 105, 96) 


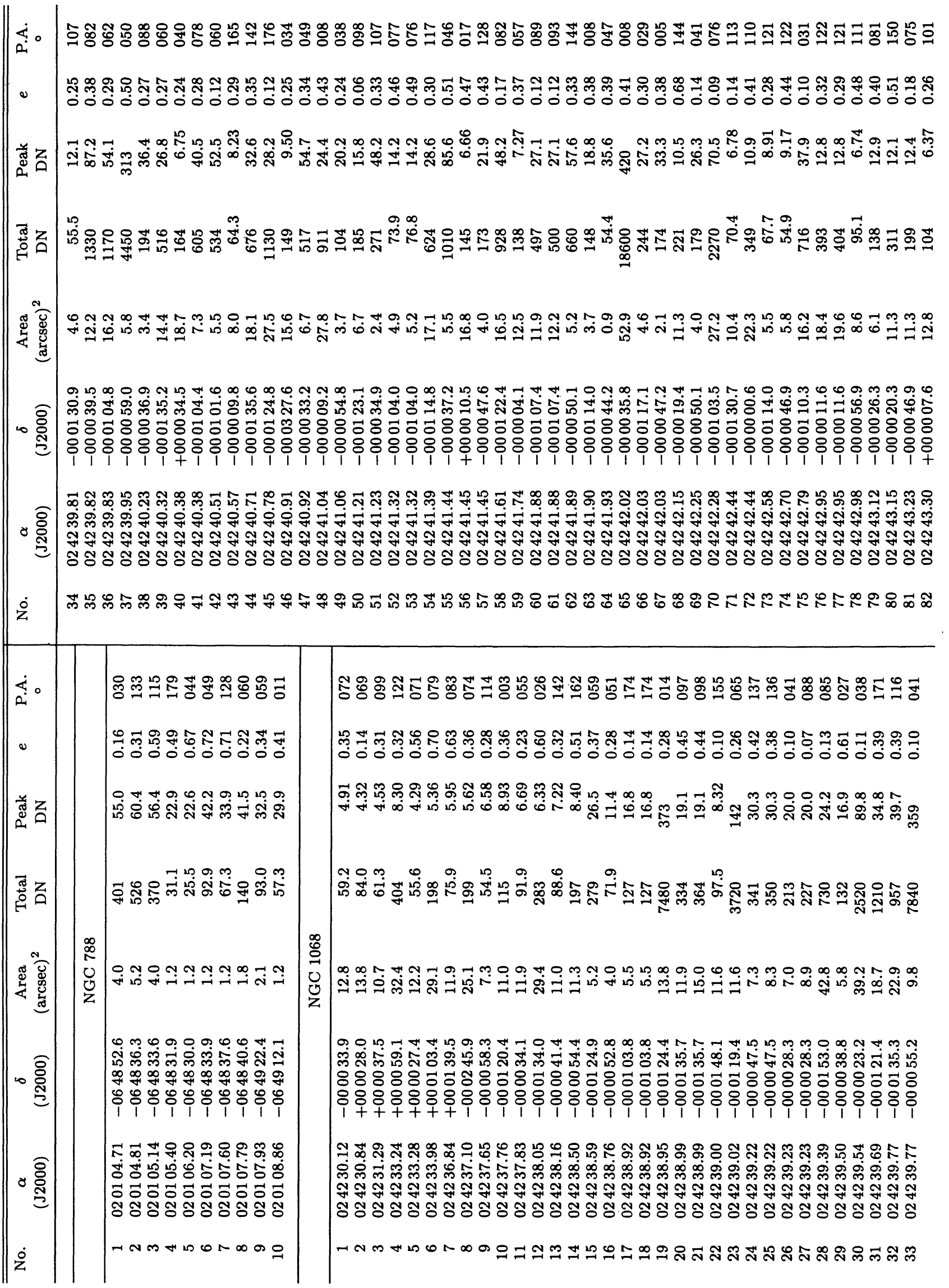




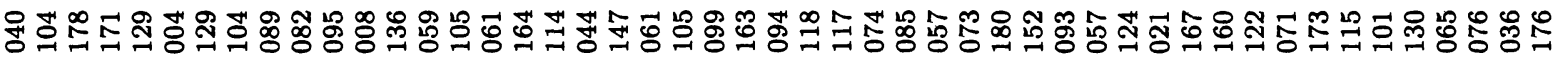

붕ํㅐ ó

ก ด 0 ก

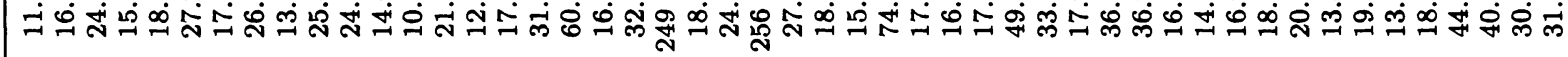

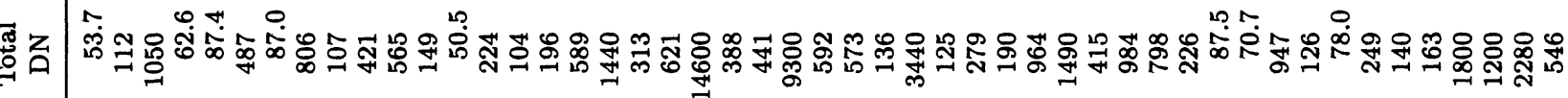

0 ด 0 N

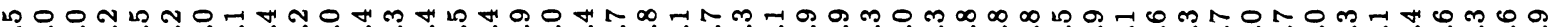
Ұ

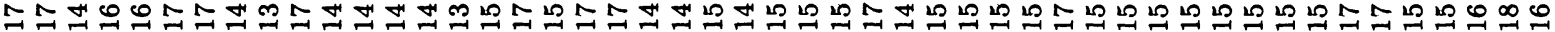

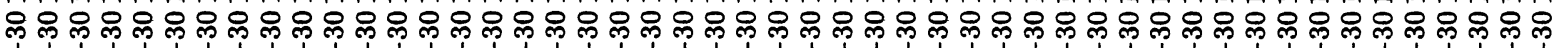

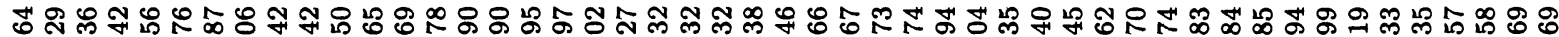

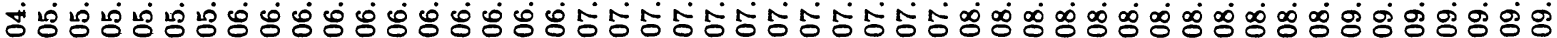

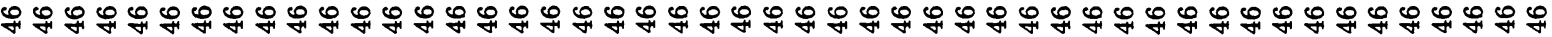

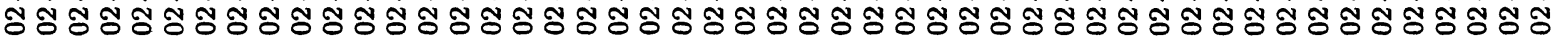

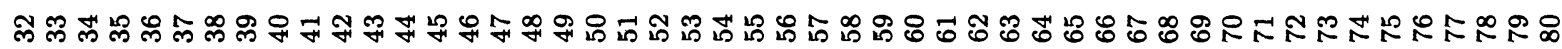

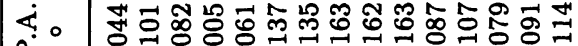

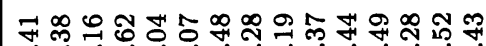
ல் $0 \dot{0} \dot{0} 0 \dot{0} \dot{0} 0 \dot{0} 0 \dot{0} 0 \dot{0}$

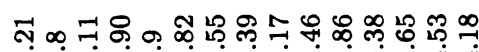

芭台 ம

戛苗

娄兽

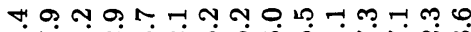
ठㅊำ

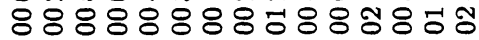

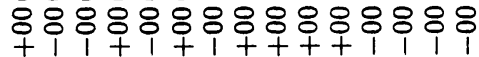

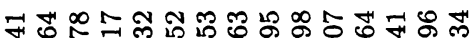

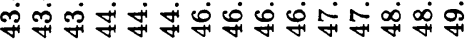

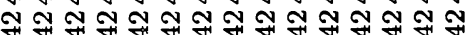

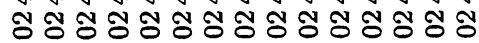

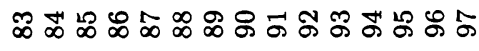

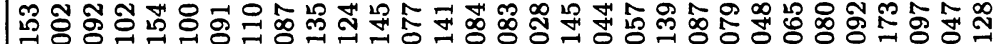

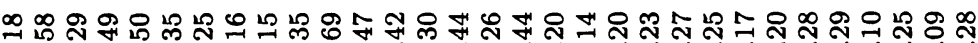

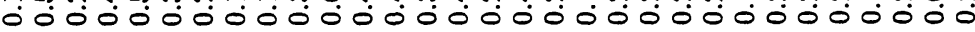

ก ๓

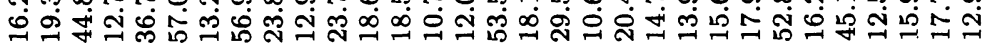

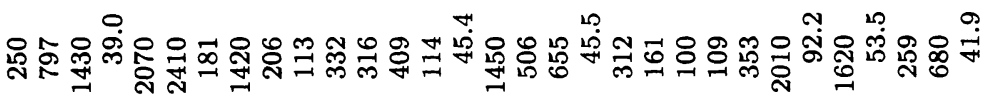

ڤ̊̀口

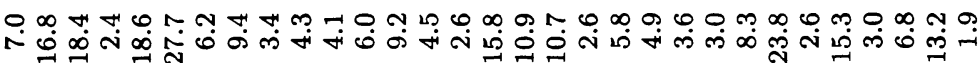

ヘ ન

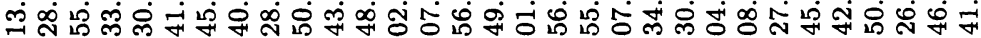

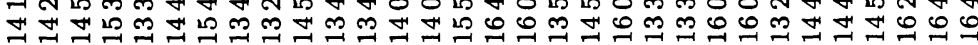

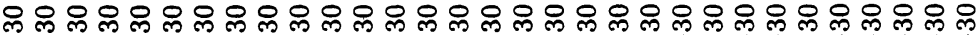

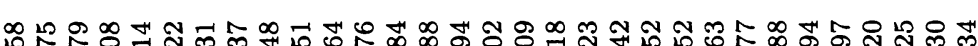

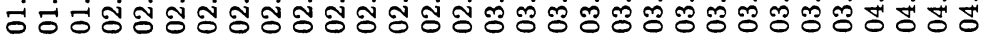

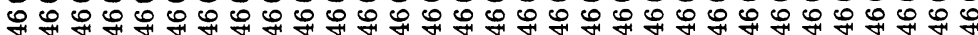

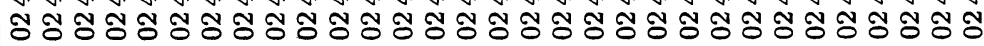

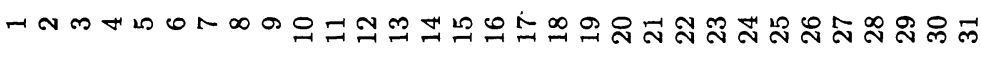




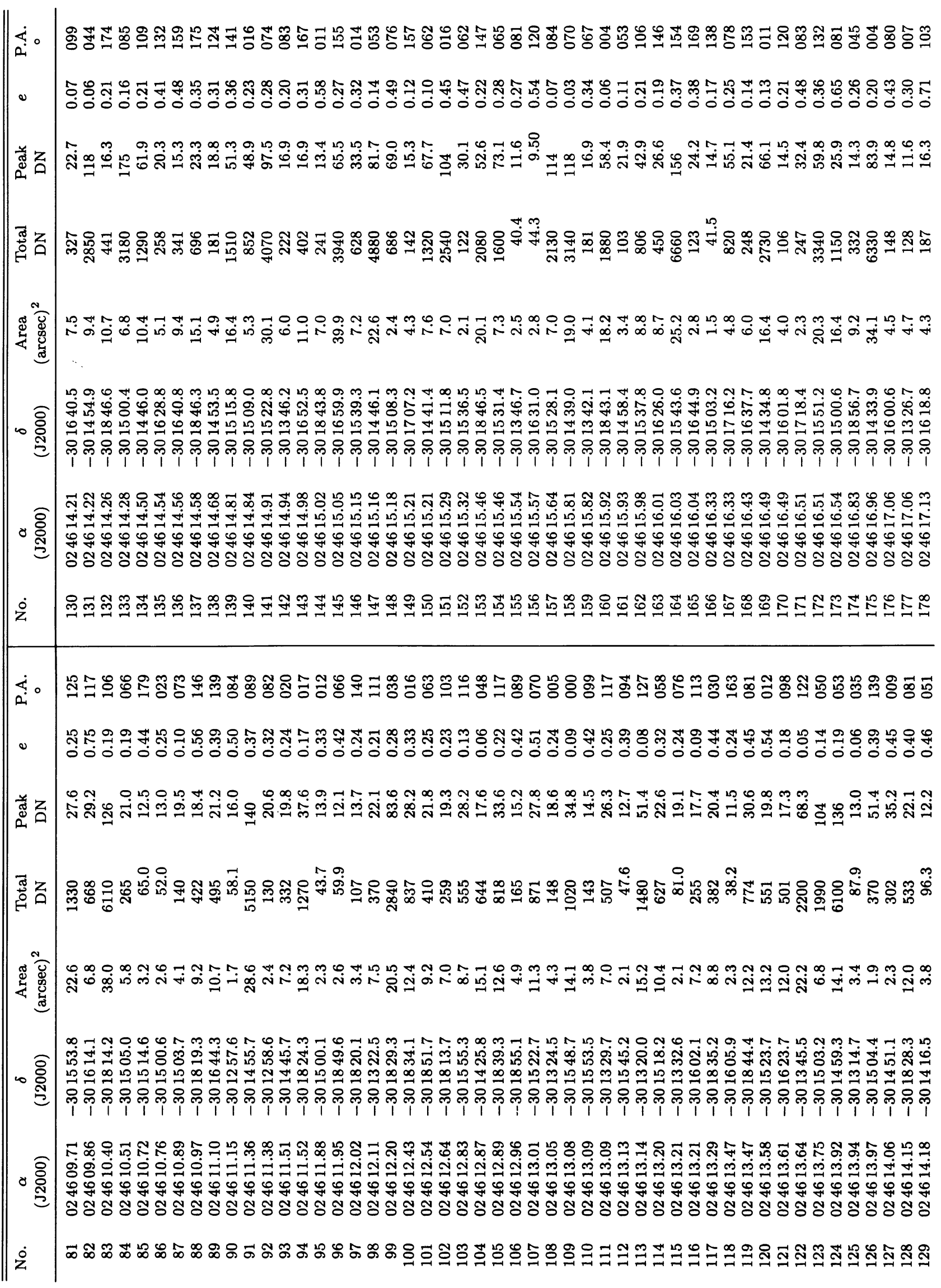




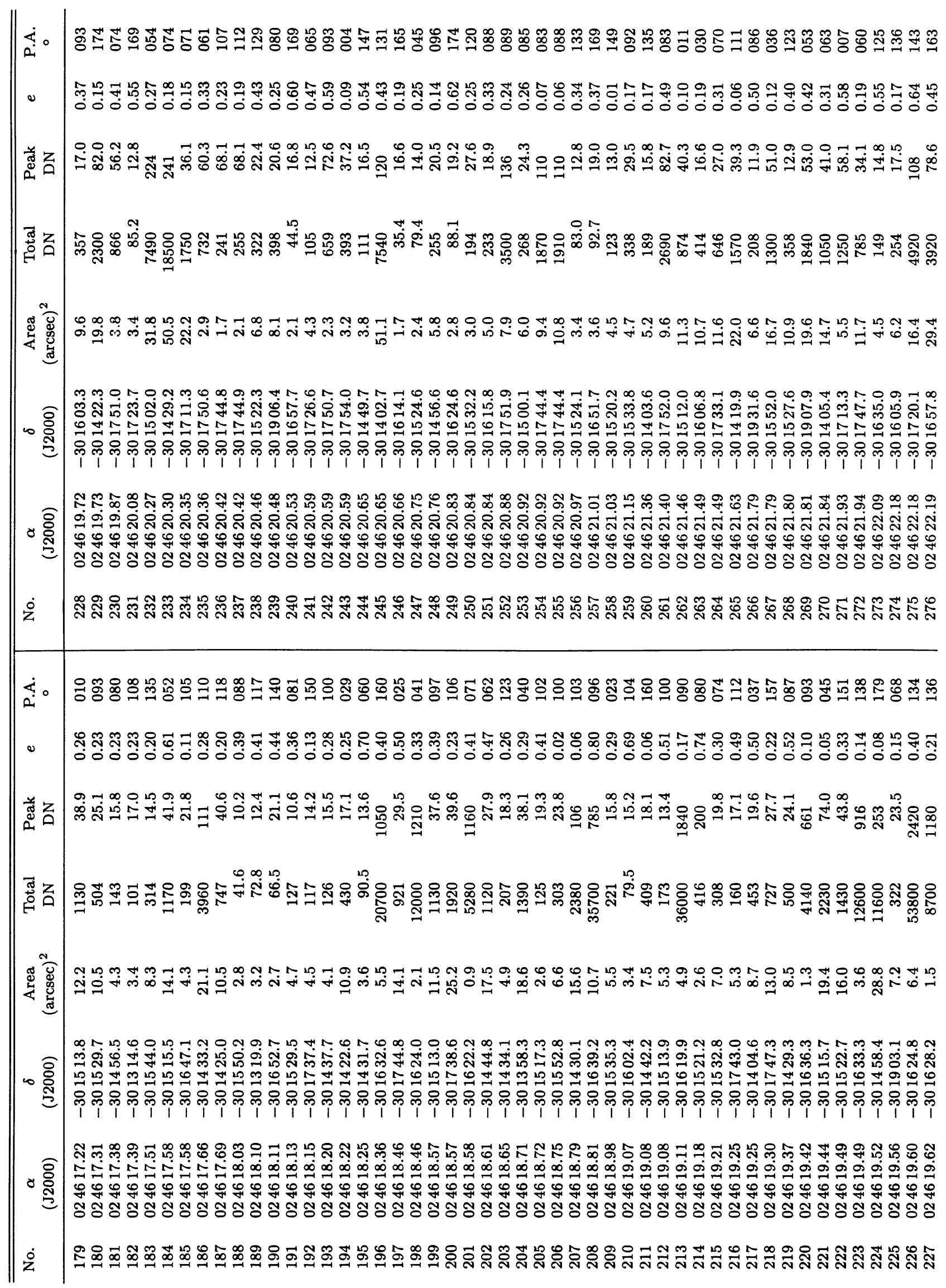




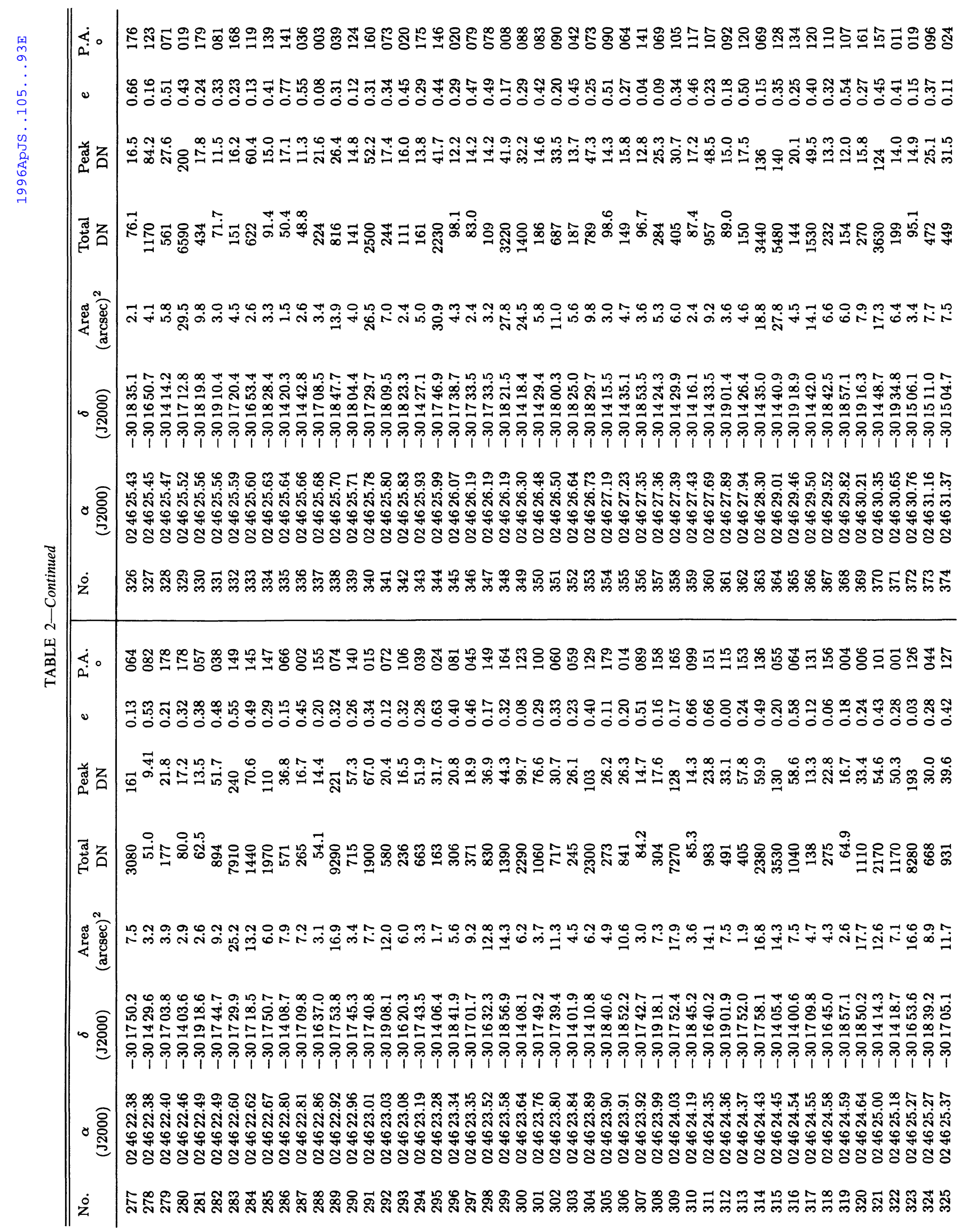




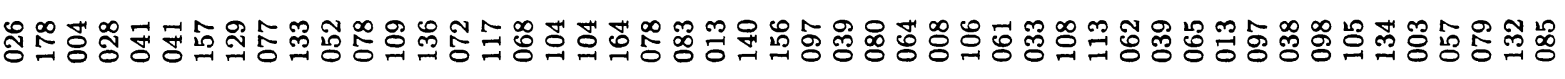

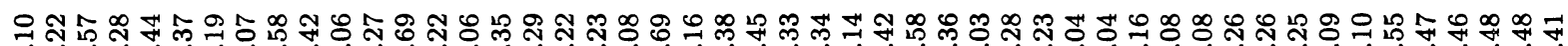

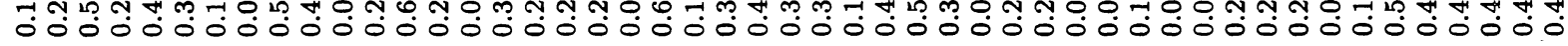

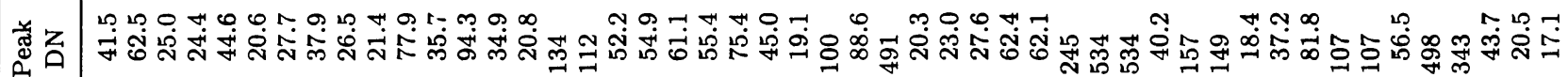

沓苗

0

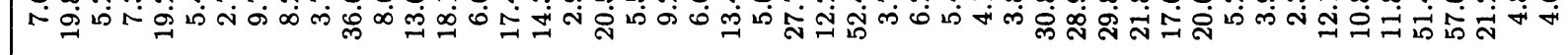

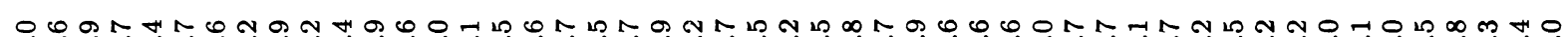
他

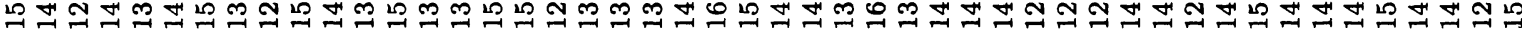

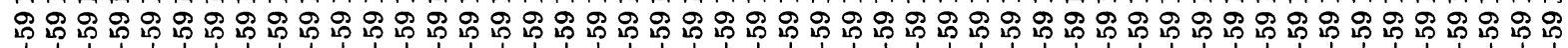

东 ฟ 连

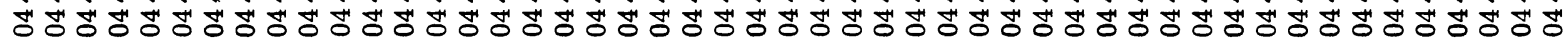

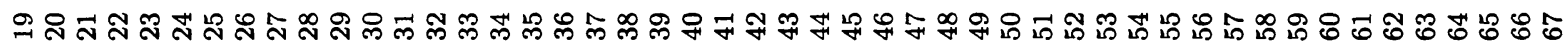

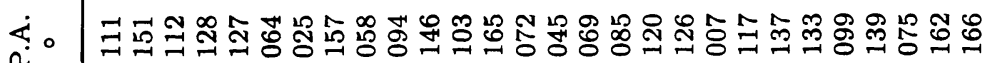

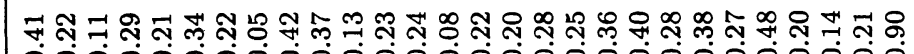

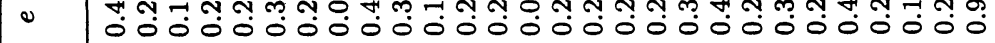

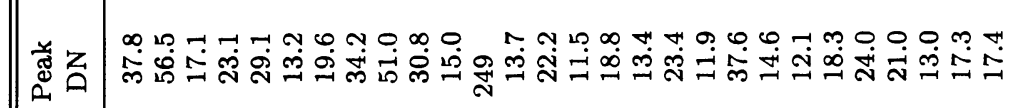

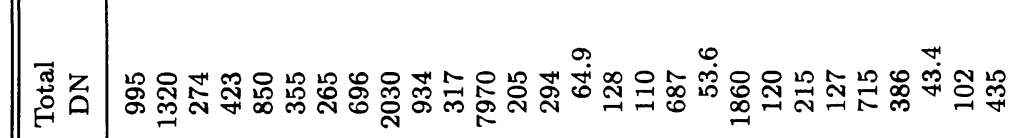

Hamo- HO mo O

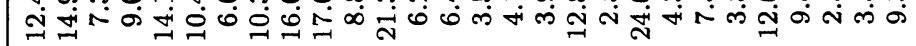

O ON⿴囗十

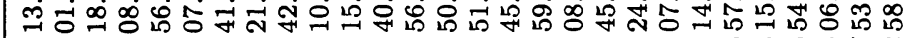

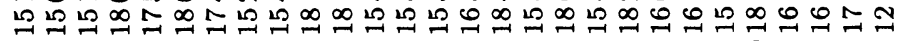

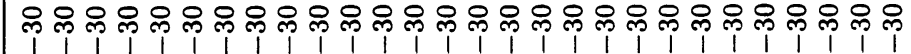

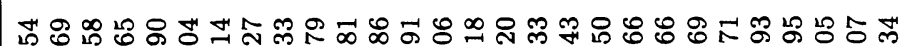

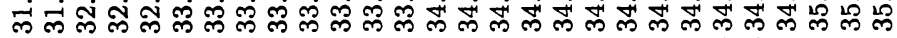

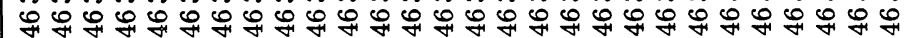

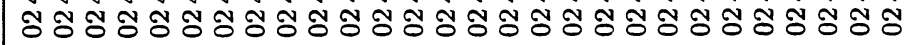

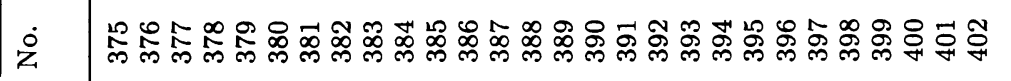

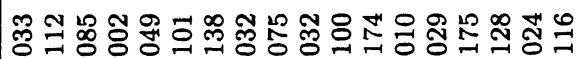

요요

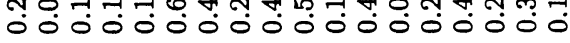

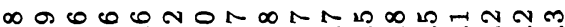
ล่ สิ

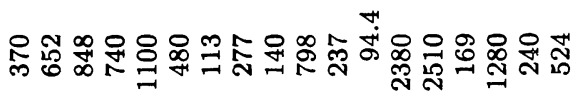

苍 U

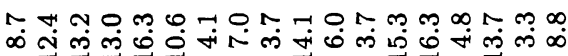

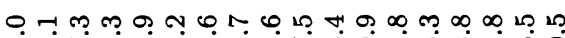

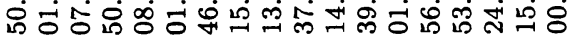

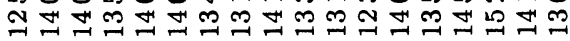

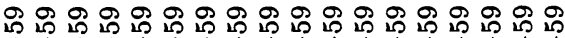

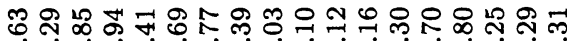
ลี่ ส่ง

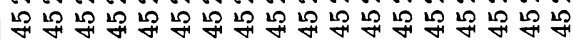

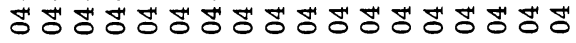

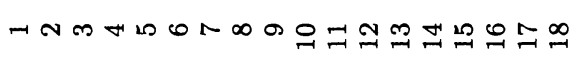




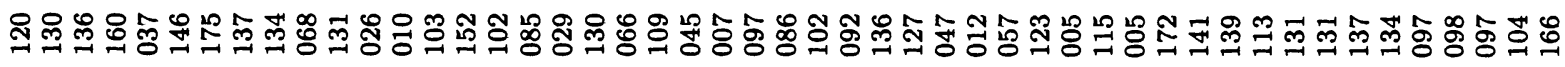

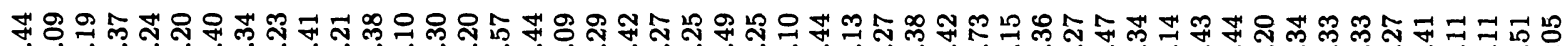


あ 勇云

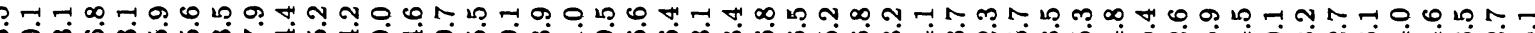

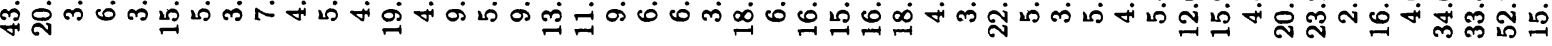

O n 9 H స్లే 政

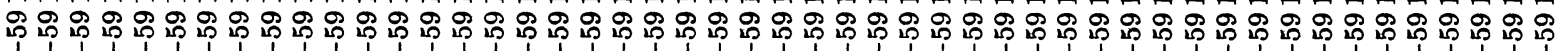
p.

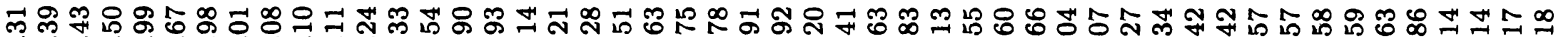

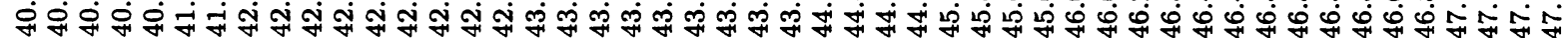

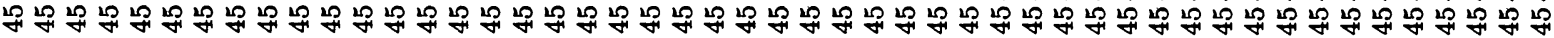

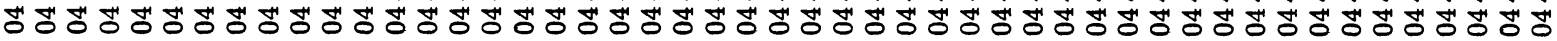

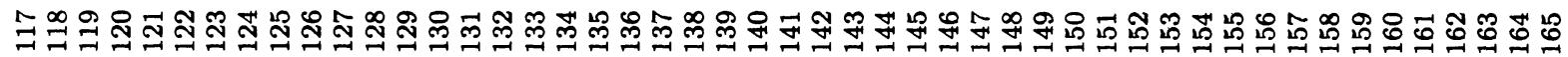

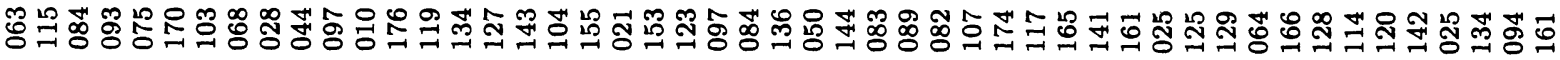

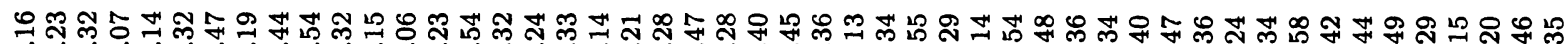

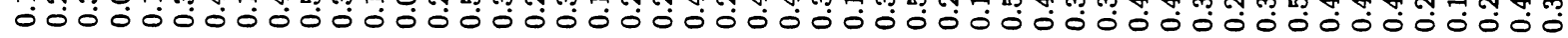
崖台 要云 ก

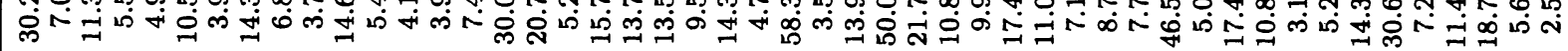

HNOGMmN H

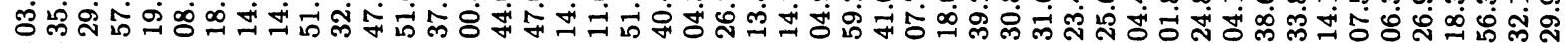

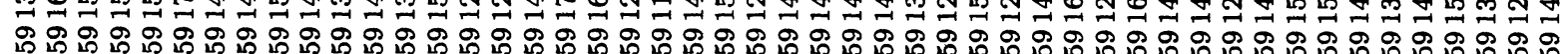

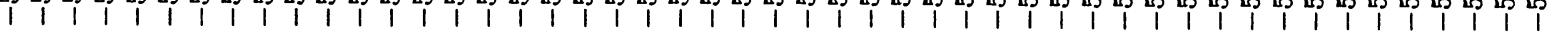

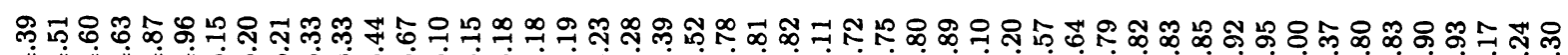

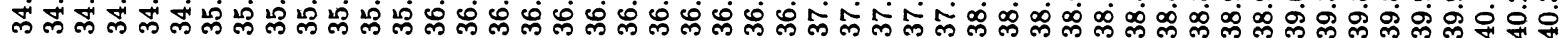

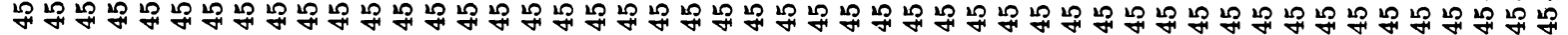

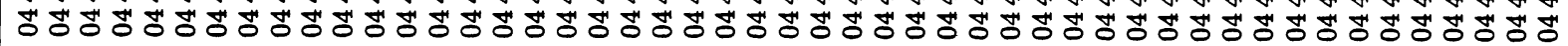




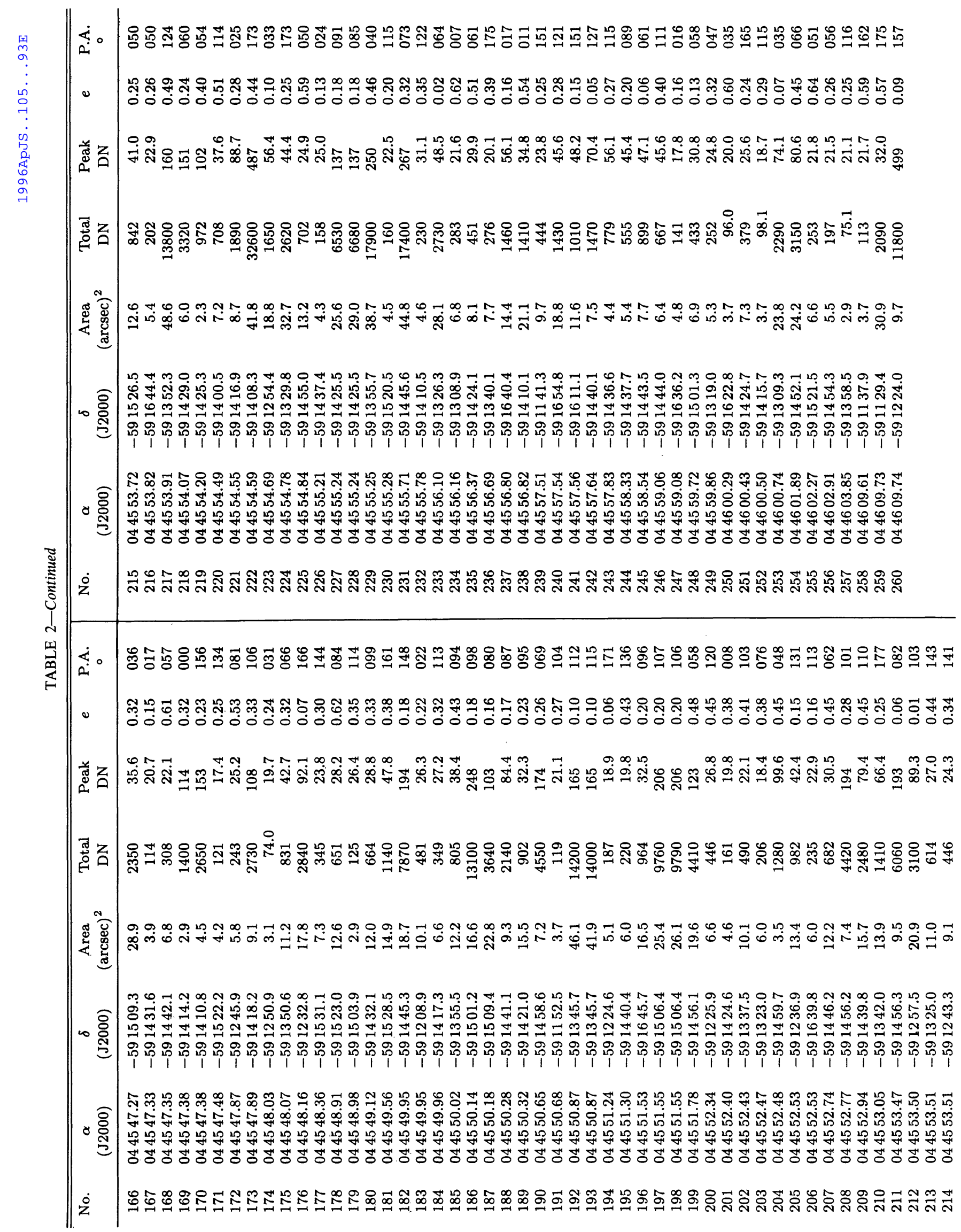




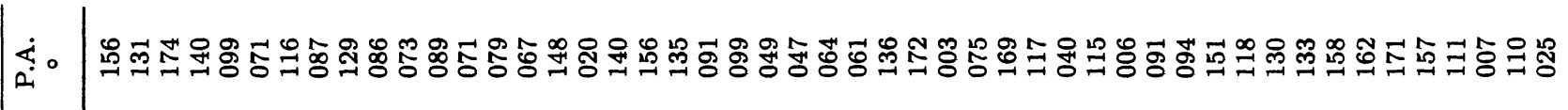

- $\quad$ m

ఇุ丷

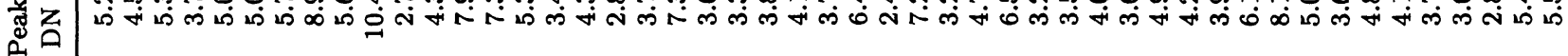

สี 荧台

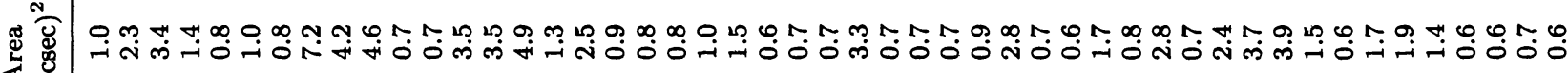
这

๘

ก ค

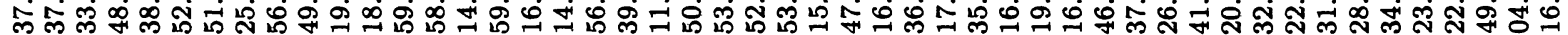

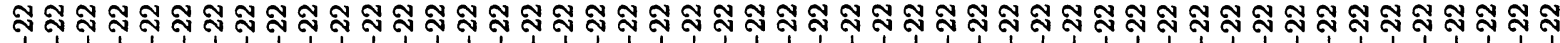

웅

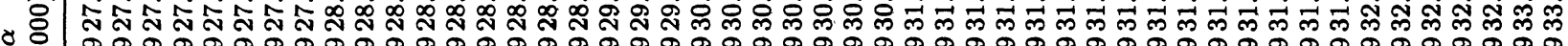

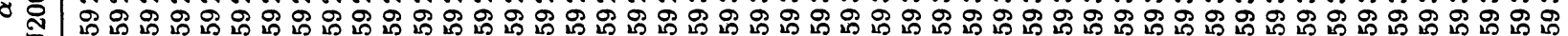
응ㅇㅇㅇㅇㅇㅇㅇㅇㅇㅇㅇㅇㅇㅇㅇㅇㅇㅇㅇㅇㅇㅇㅇㅇㅇㅇㅇㅇㅇㅇㅇㅇㅇㅇㅇㅇㅇㅇㅇㅇㅇㅇㅇㅇㅇㅇㅇㅇㅇㅇㅇㅇㅇㅇㅇㅇㅇㅇㅇㅇㅇㅇㅇㅇㅇㅇㅇㅇㅇㅇㅇㅇㅇㅇㅇ

ปั. $\dot{2}$

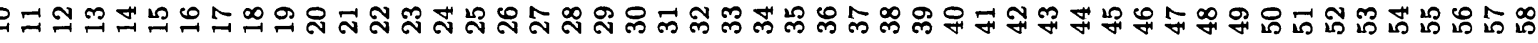

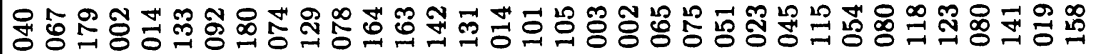

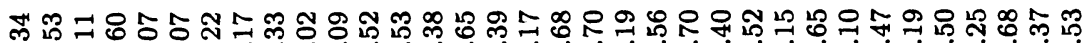

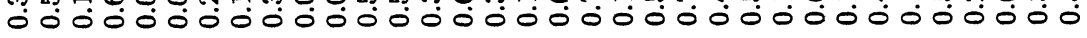

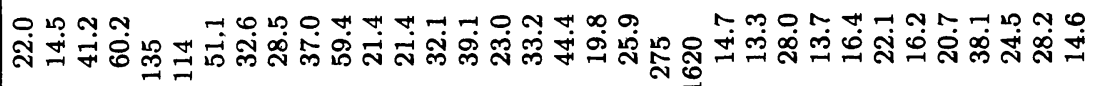
旁云 疍台 每 ֻ

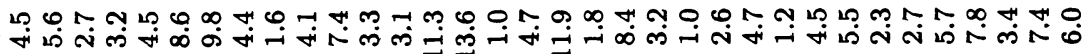

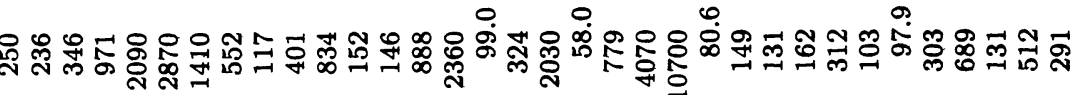

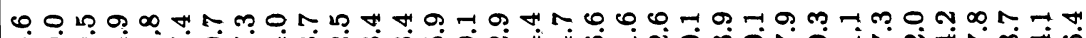
ம்

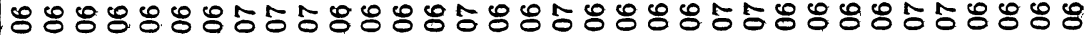

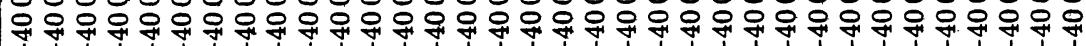
t $t+t+t+t+t+t+t+t+t+t+t+t+t+t+t+t+t+$

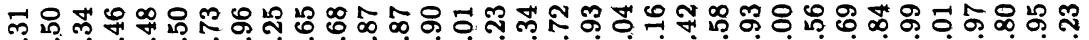
ப்

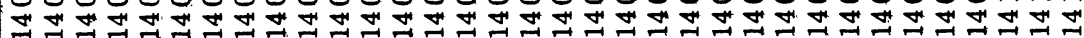
용ㅇㅇㅇㅇㅇㅇㅇㅇㅇㅇㅇㅇㅇㅇㅇㅇㅇㅇㅇㅇㅇㅇㅇㅇㅇㅇㅇㅇㅇㅇㅇㅇㅇㅇㅇㅇㅇ ーヘッナน

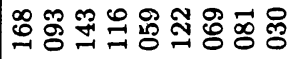

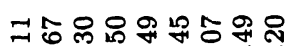

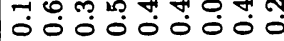

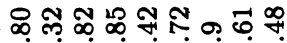

ن்

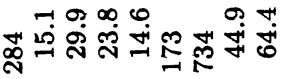

总

m 0 HON

o)

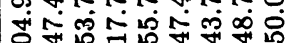

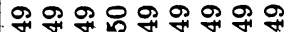
สิ ส ส 1 1$\}, 1,1$

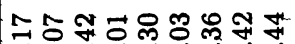
๓ึ่มี่

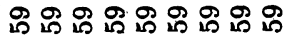
용ㅇㅇㅇㅇㅇㅇㅇ 


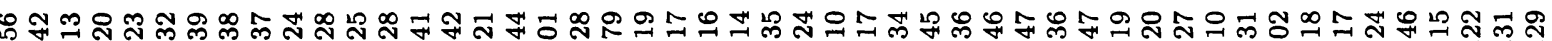
$\infty m N-m N$ N

.




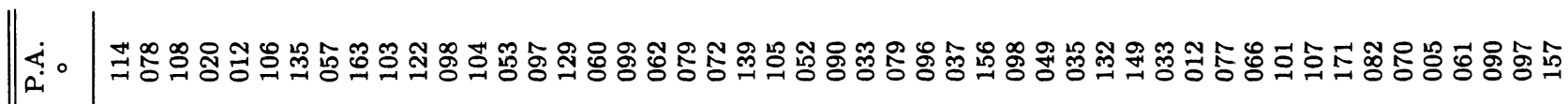

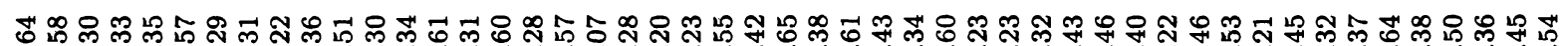

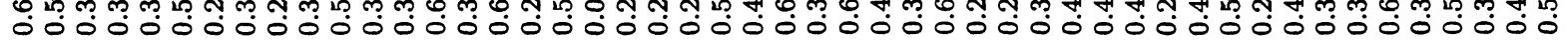
范z

焉台

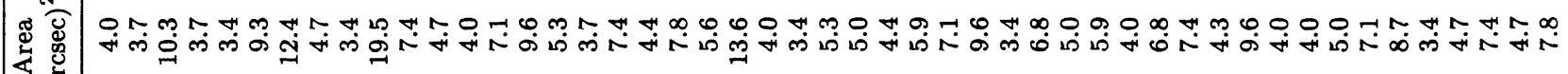

م

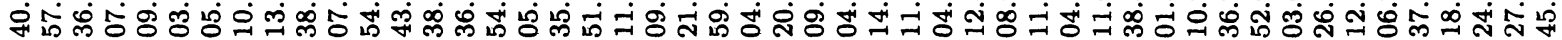

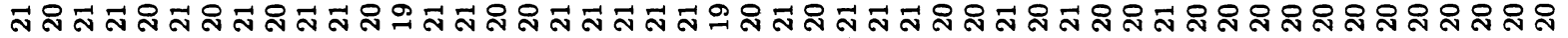

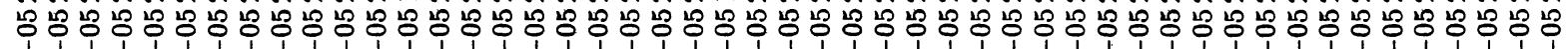

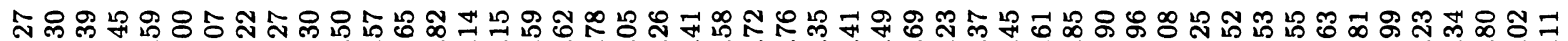

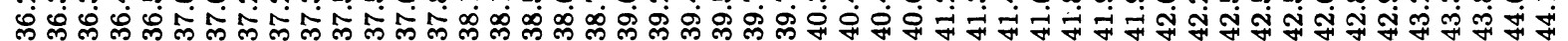

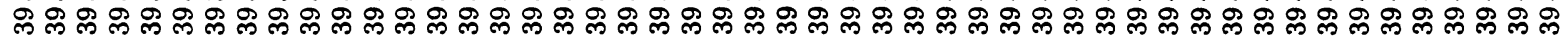

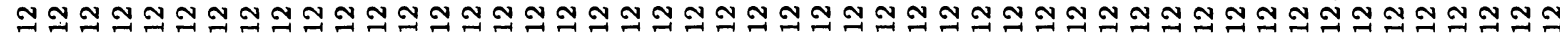

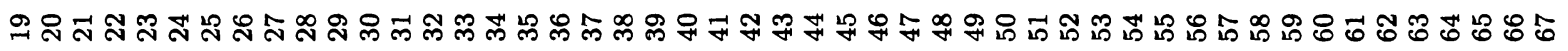

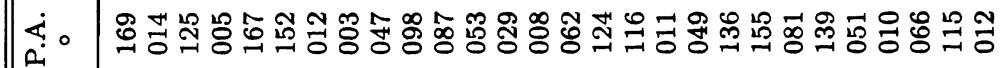

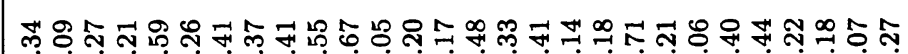

苛苗

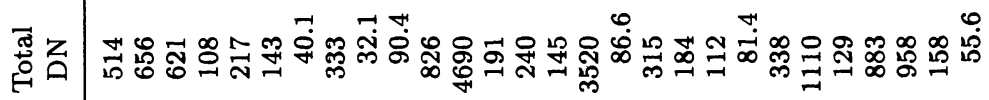

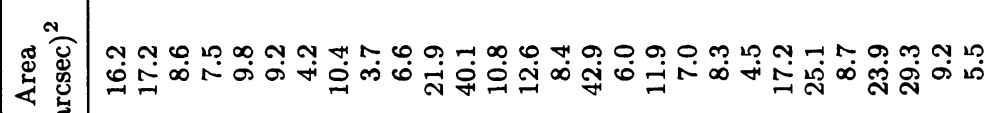

N

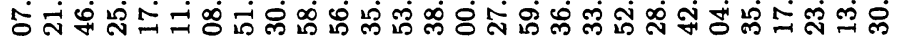

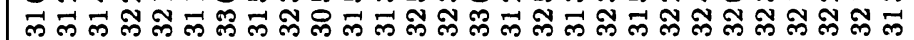

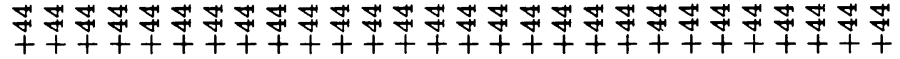

ஜ

12

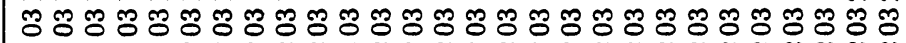

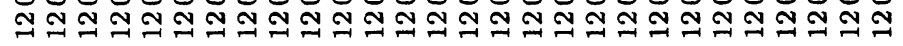

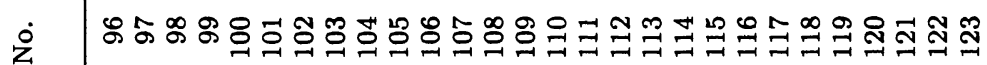

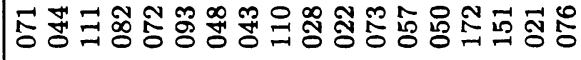

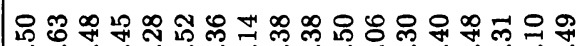
O000000000000000000

ー

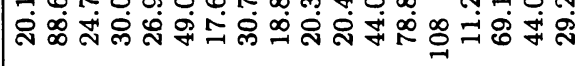

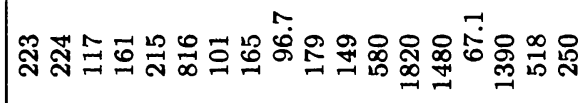

常

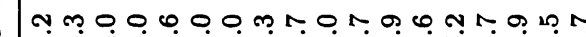
כु

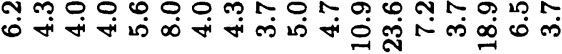

Nล m

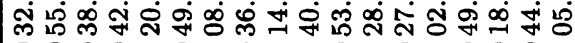

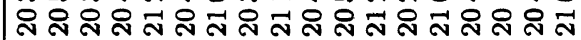

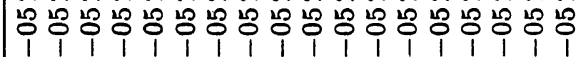

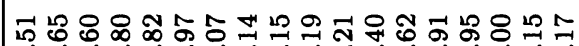

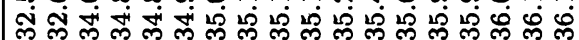

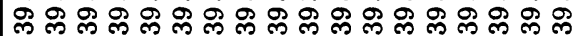

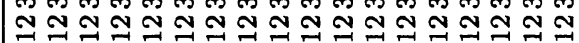

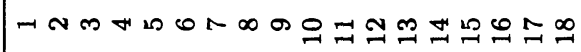




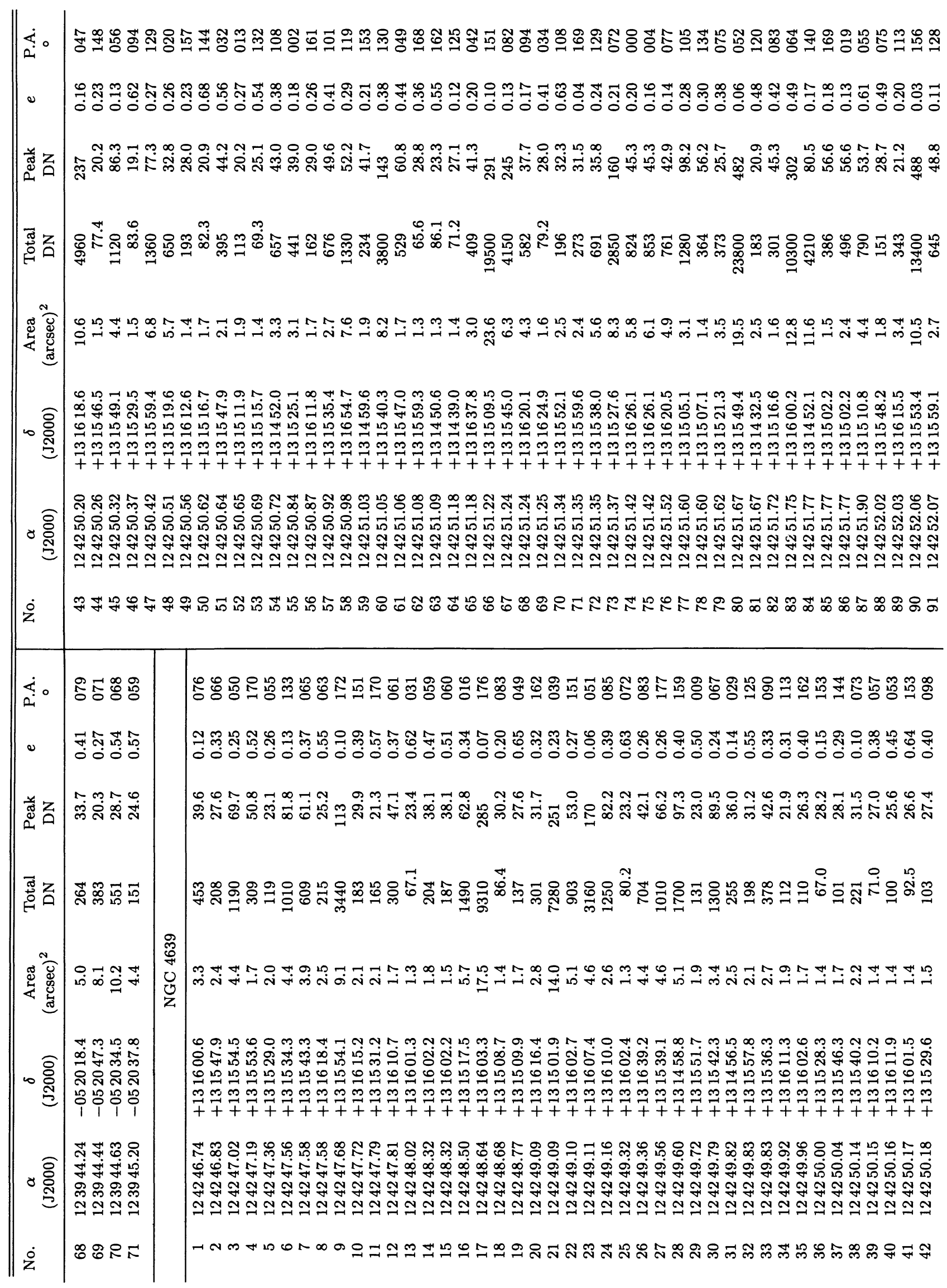




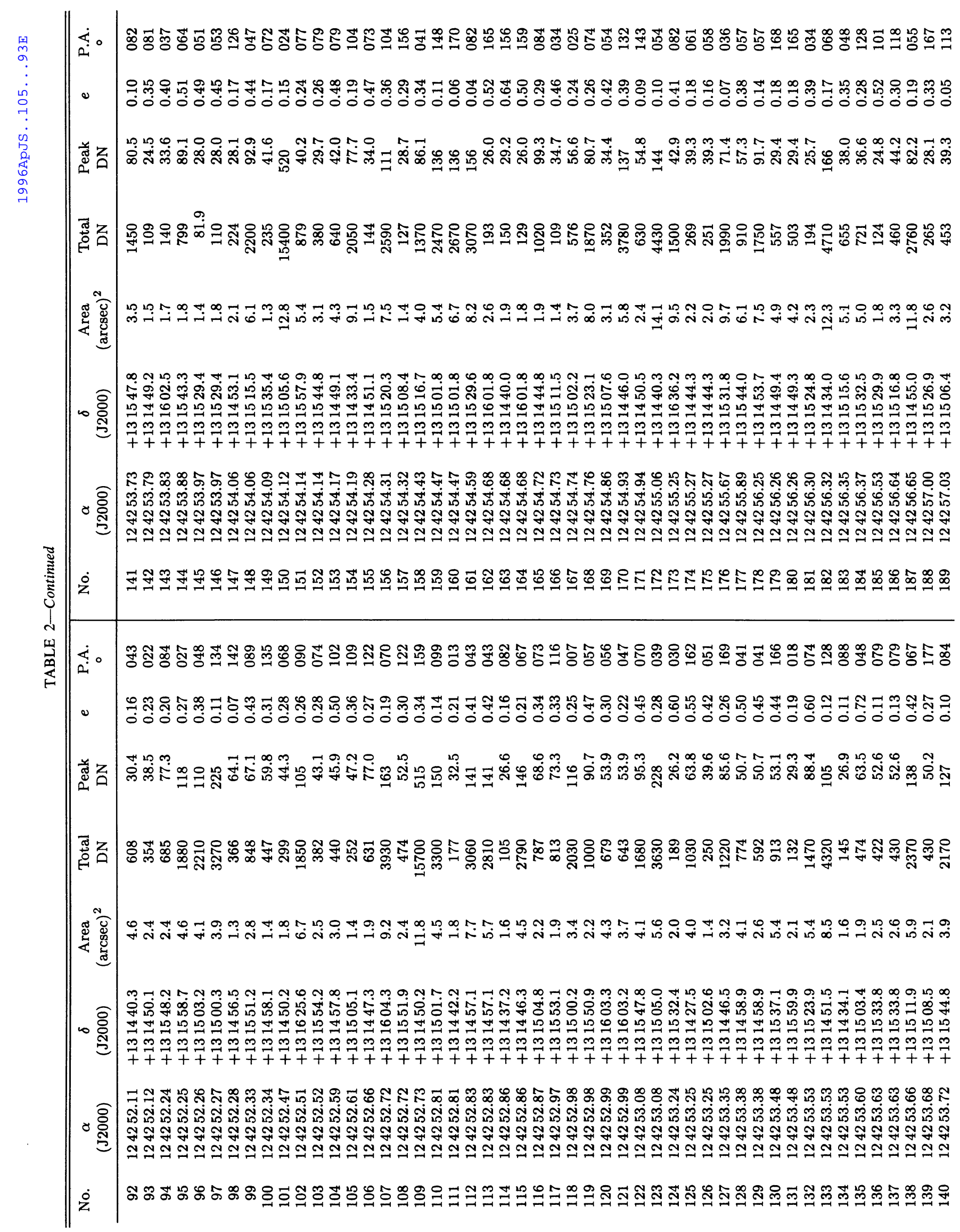




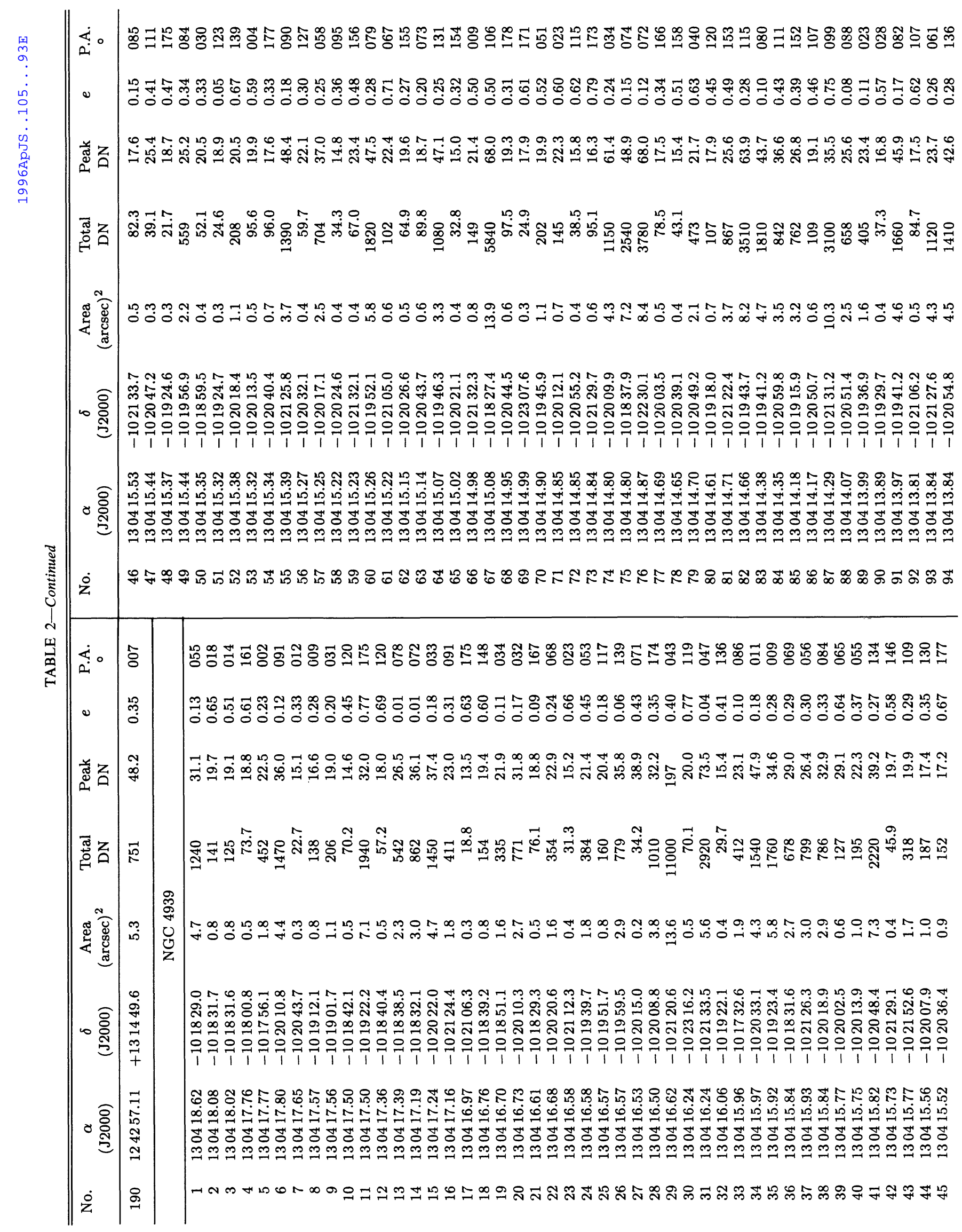




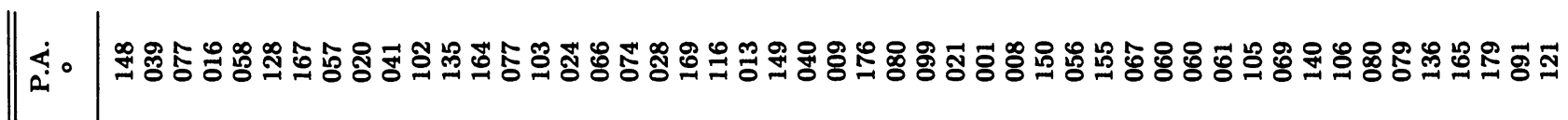

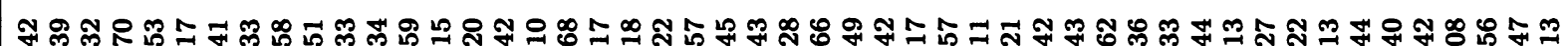

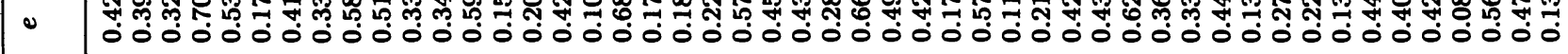

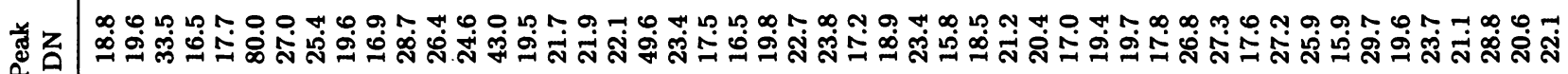

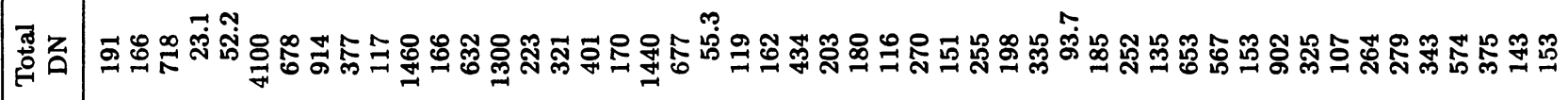

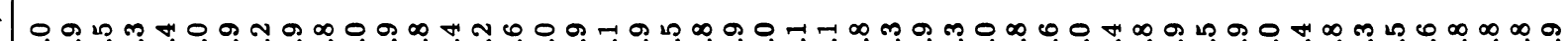
造惫

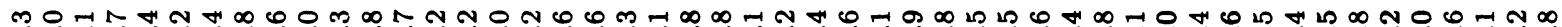

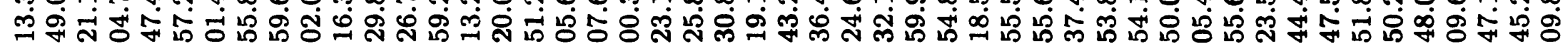

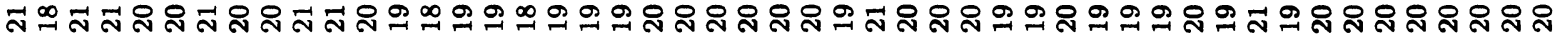

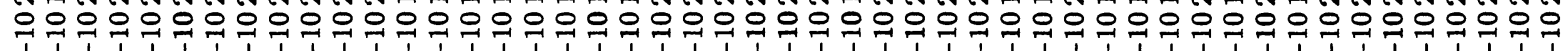

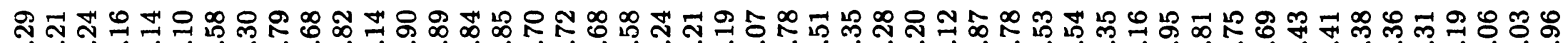
○ิ

ช 에

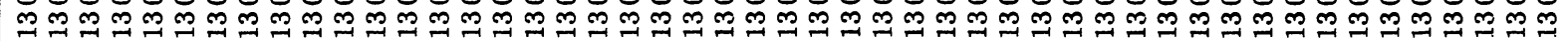

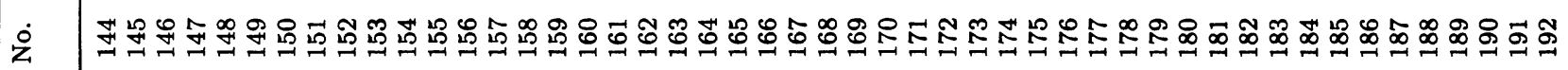

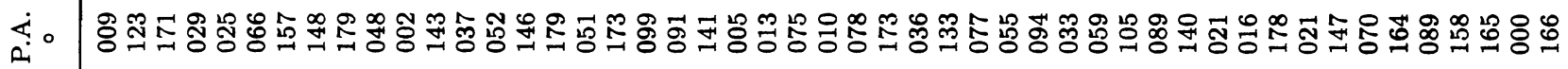

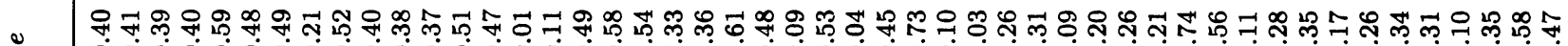
0000000000000000000000000000000000000000000000000

范奋

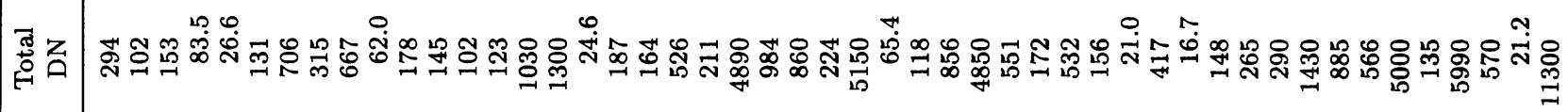

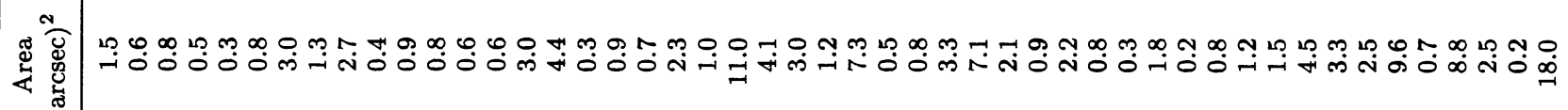

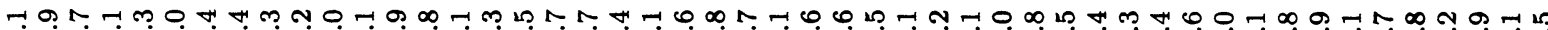

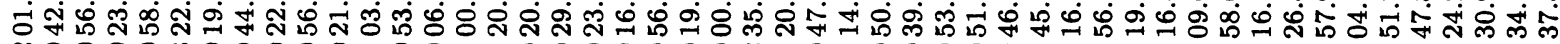

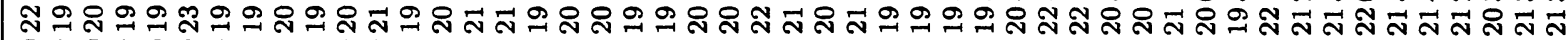

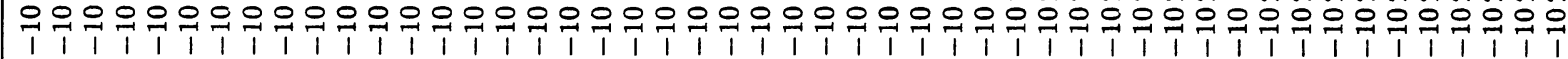

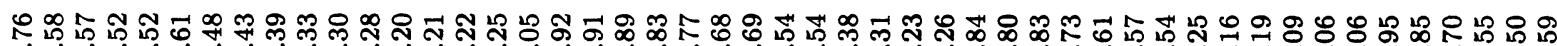

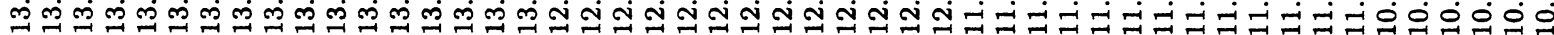

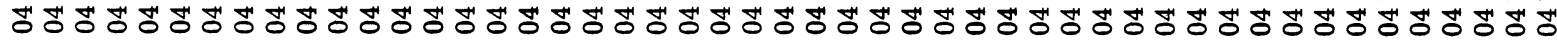

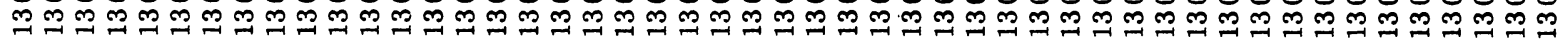

\& \& 


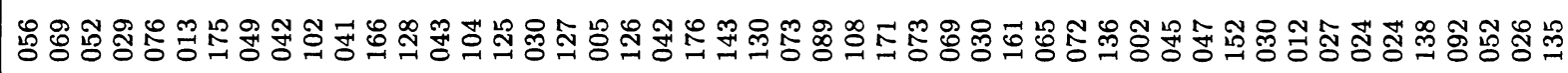

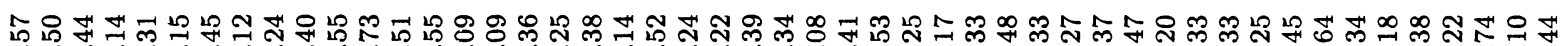

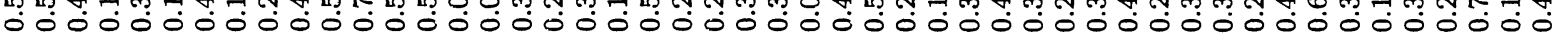

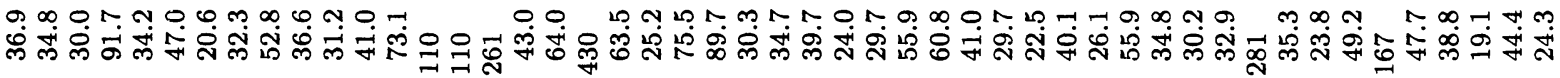

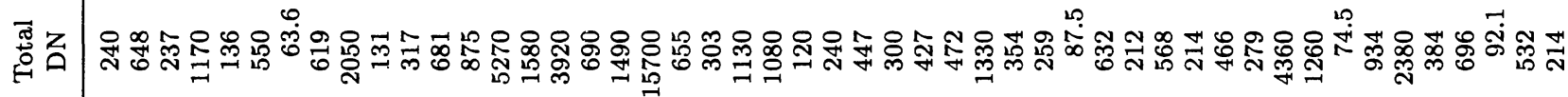

N 0 L 0 0 0 H N ON-

๓ N ஸें

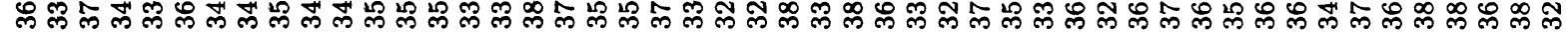

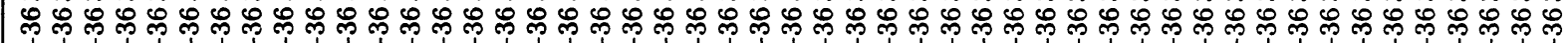

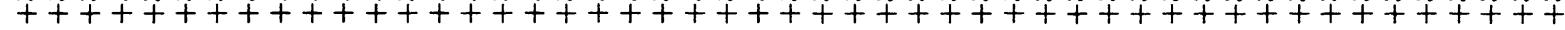

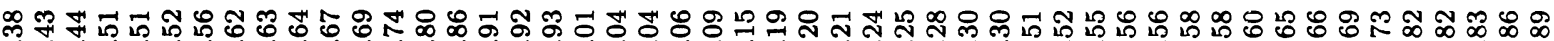

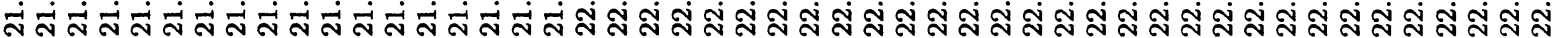

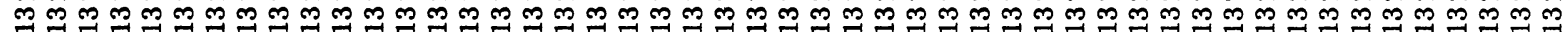

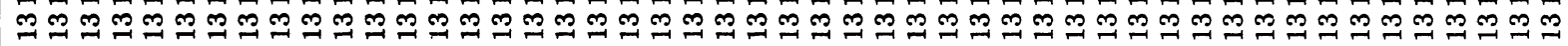

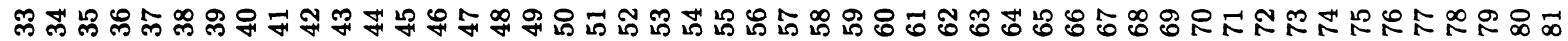

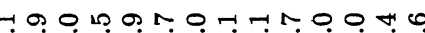

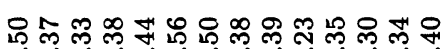

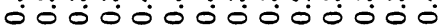

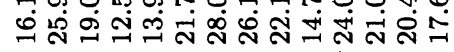

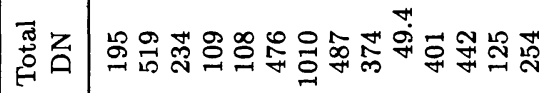

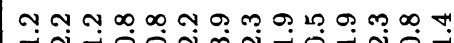

Dog

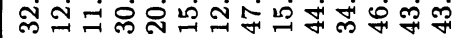

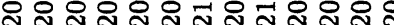

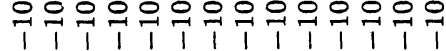

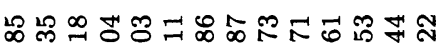

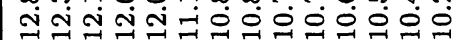

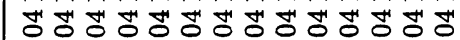
m

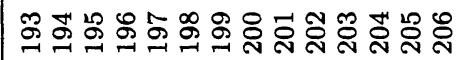

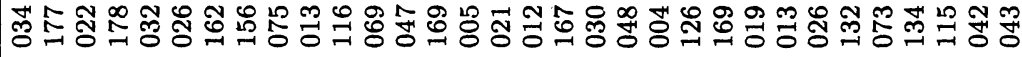

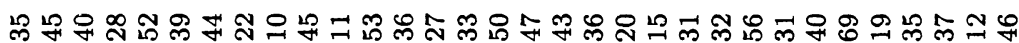

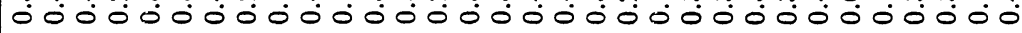

†

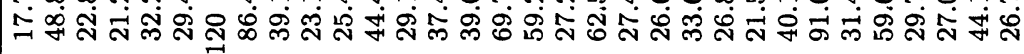

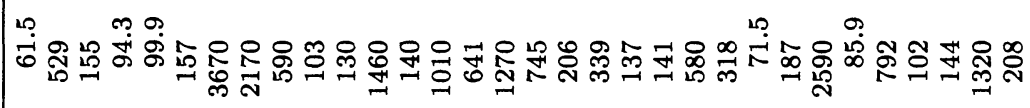

ஜூ:

近 乙

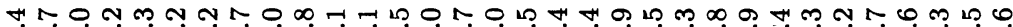

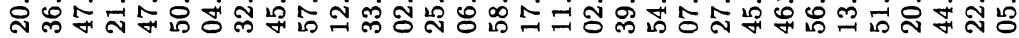

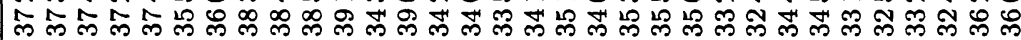
庆

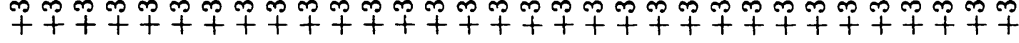

컷ำ

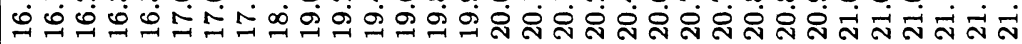

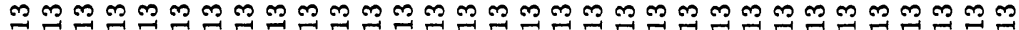

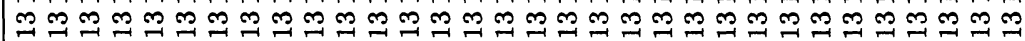

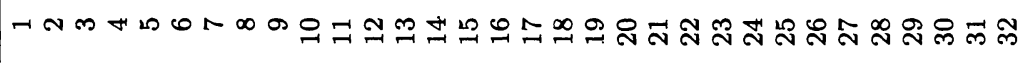




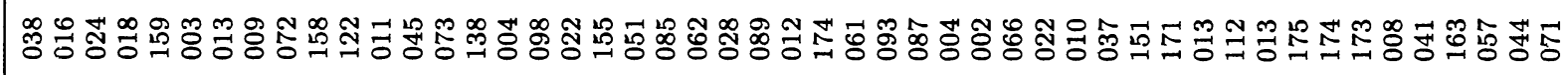

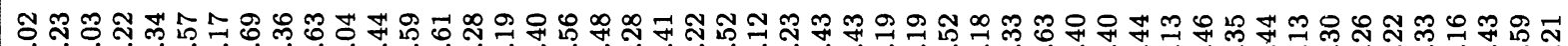

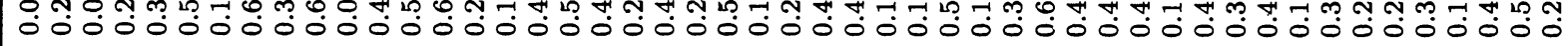

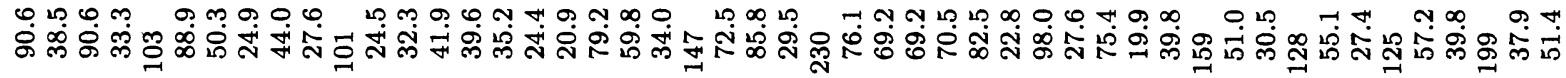

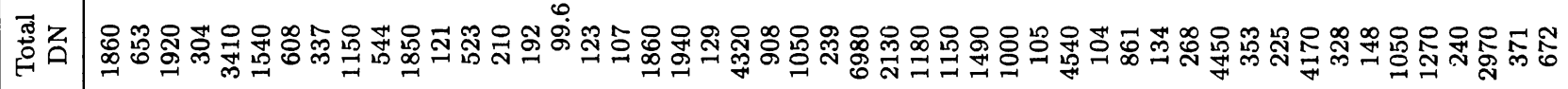

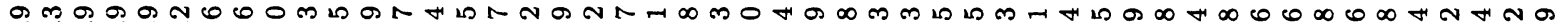
๒

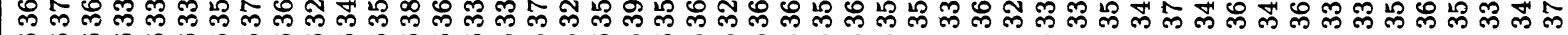

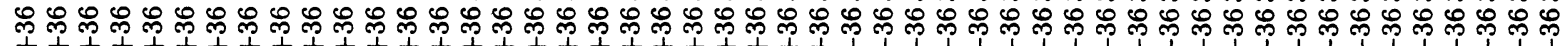
$+t+t+t+t+t+t+t+t+t+t+t+t+t+t+t+t+t+t+t+t+t+t+t+t+$

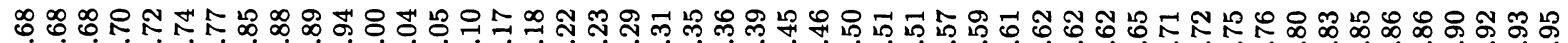

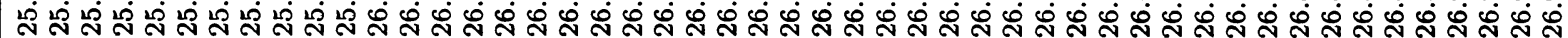

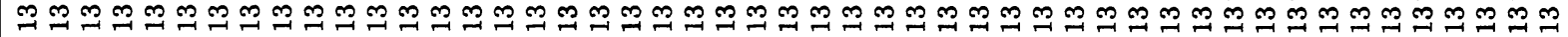

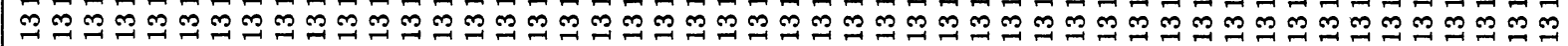

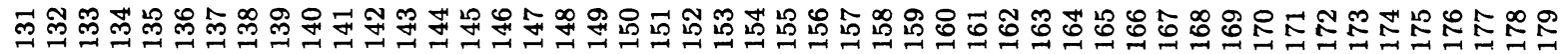

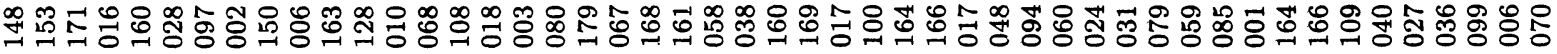

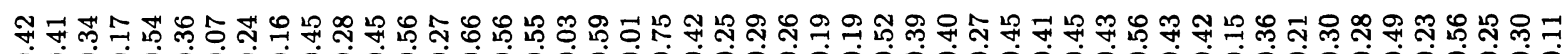
00000000000000000000000000000000000000000000000000

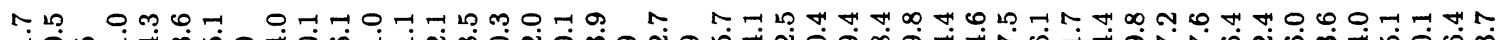

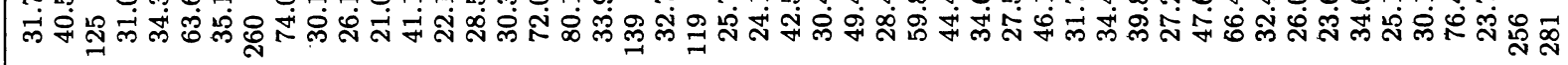

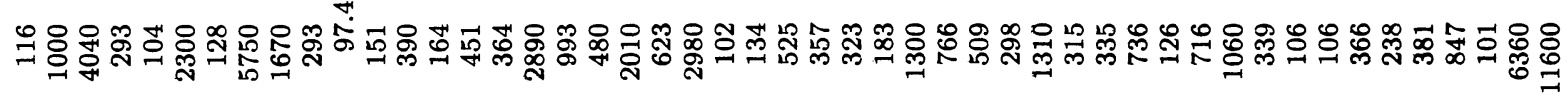

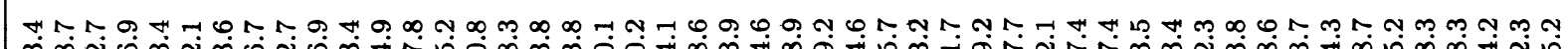

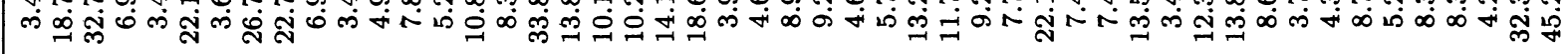

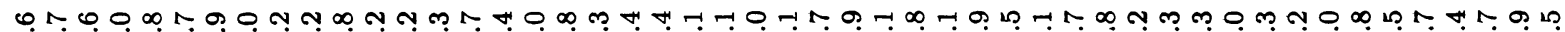

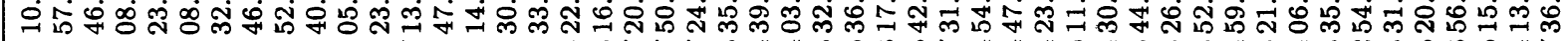

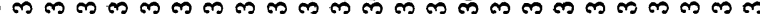

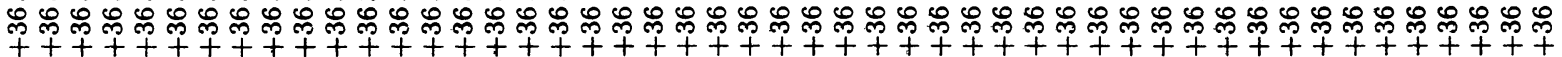

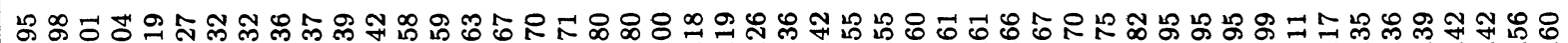
สู่ $m m m m m m m m m m m m m m m m m m m m m m m m m m m m m m m m m m m m m m$

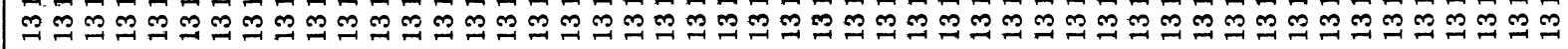

வ 


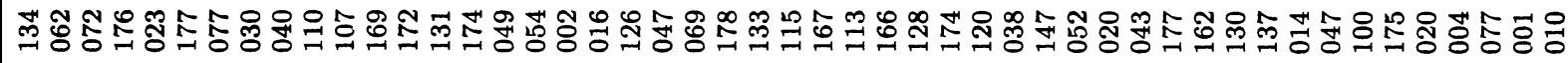

ణ

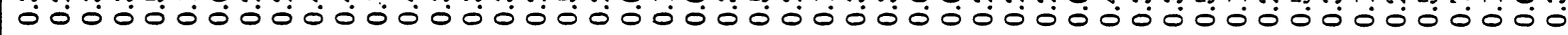

क

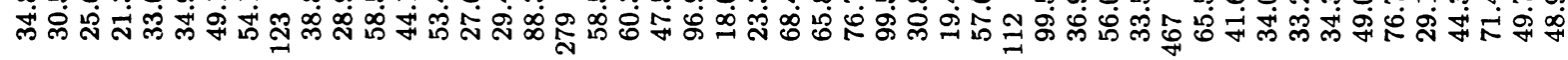

敢苜

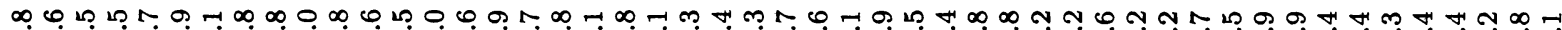
ฮี

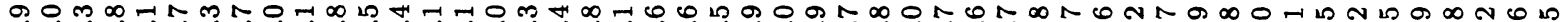

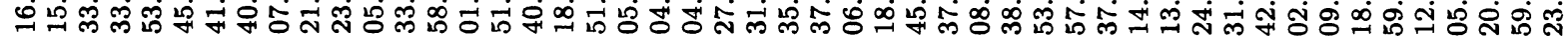

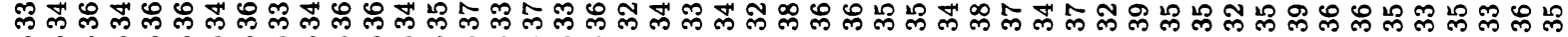

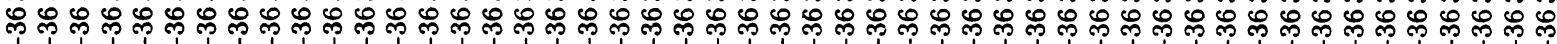

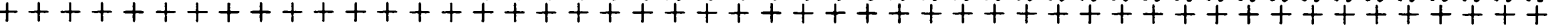

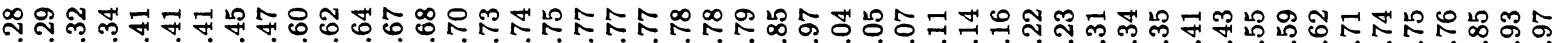
i্ m

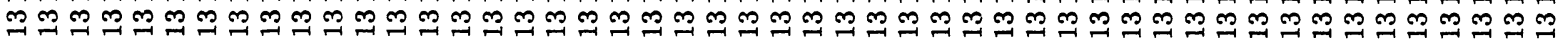

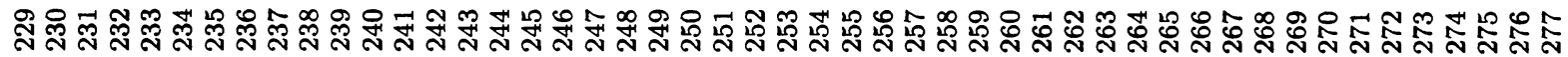

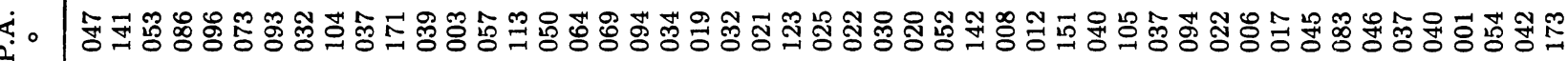

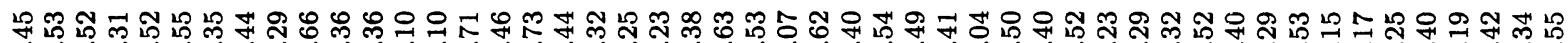
0000000000000000000000000000000000000000000000000

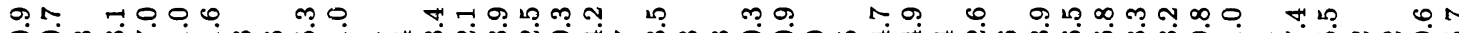

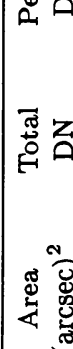

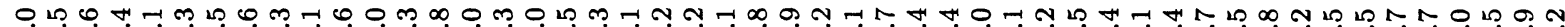

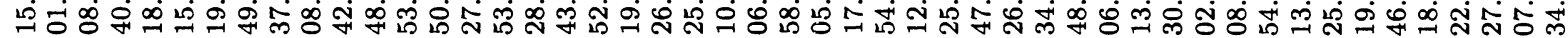

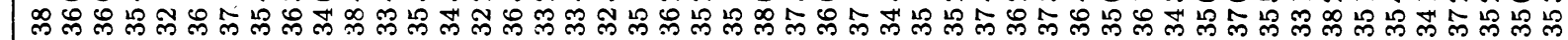

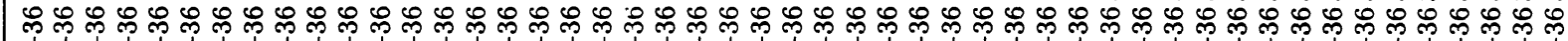

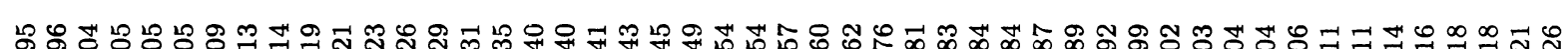

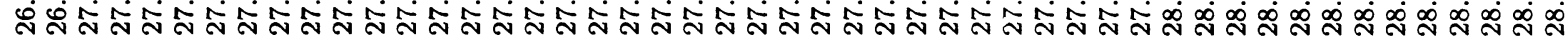

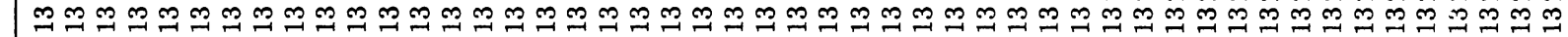

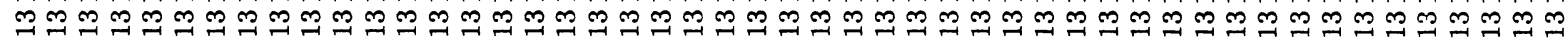

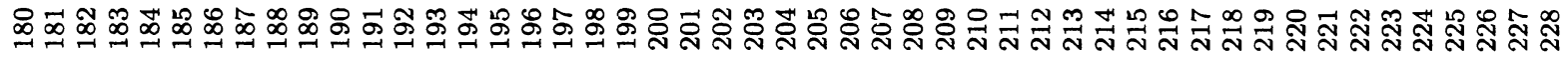




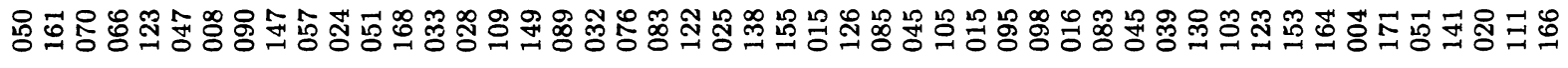

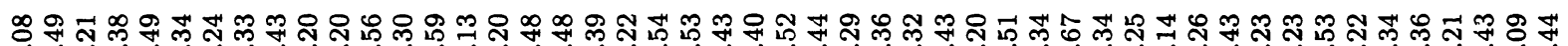

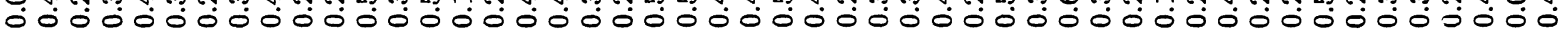

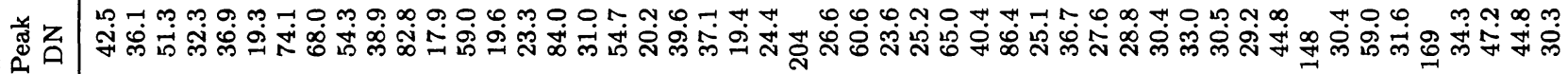

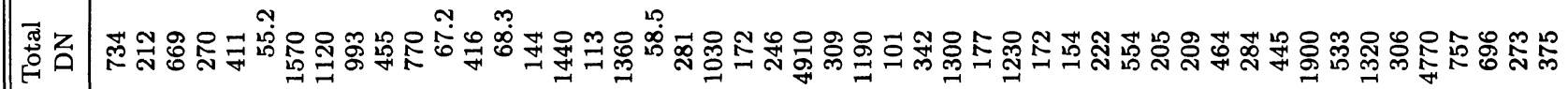

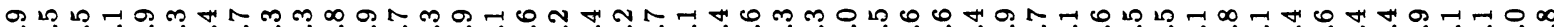

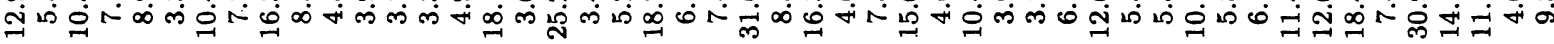

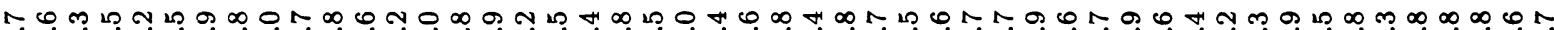

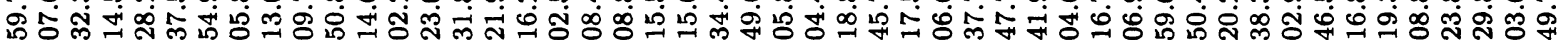

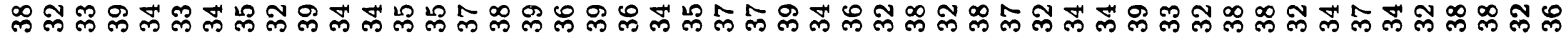

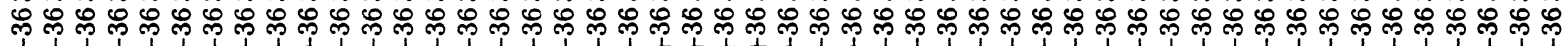

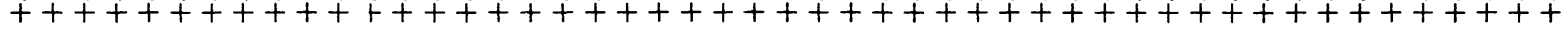

舟 굴

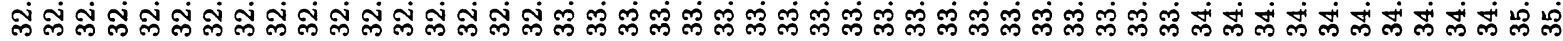

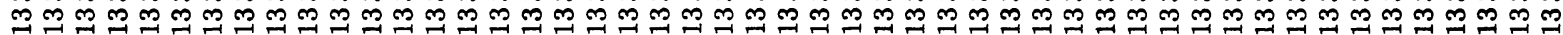

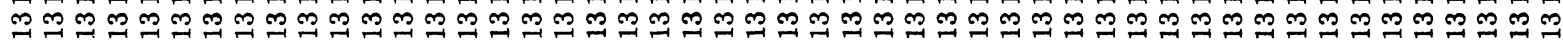

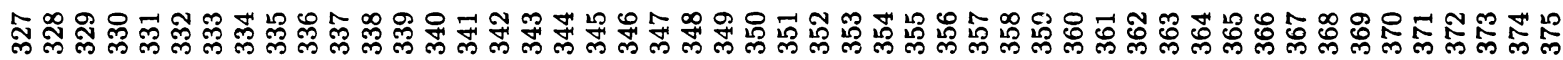

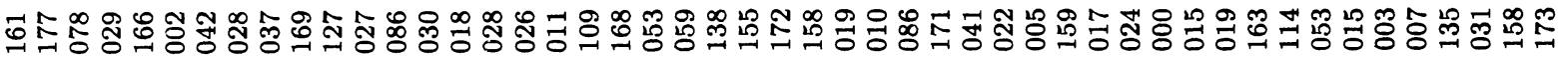

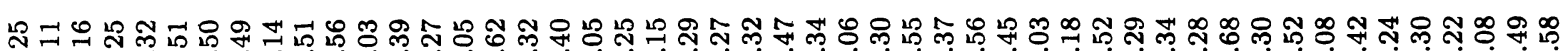

- $\quad$ o

范云

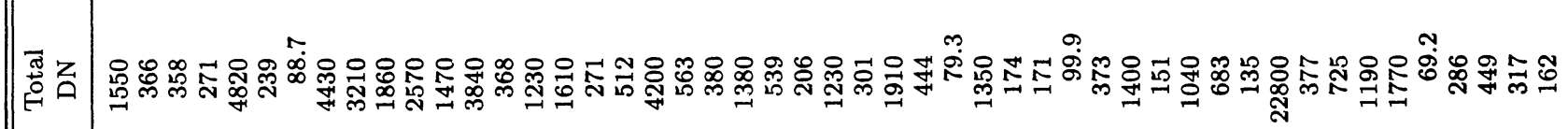
选焉

MNN H T H N N ம்

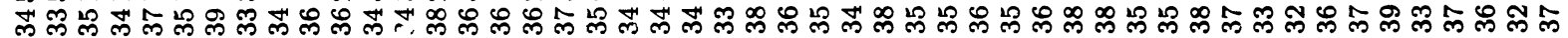
员

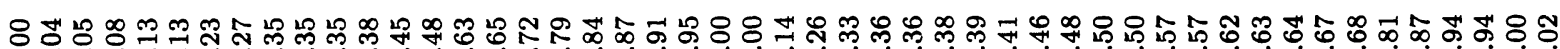

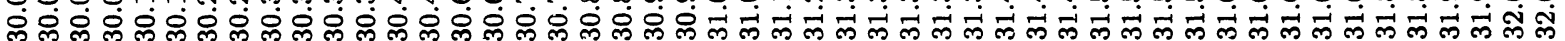
m

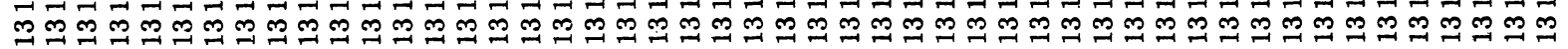

| $\quad$ 它 $\quad$ N 


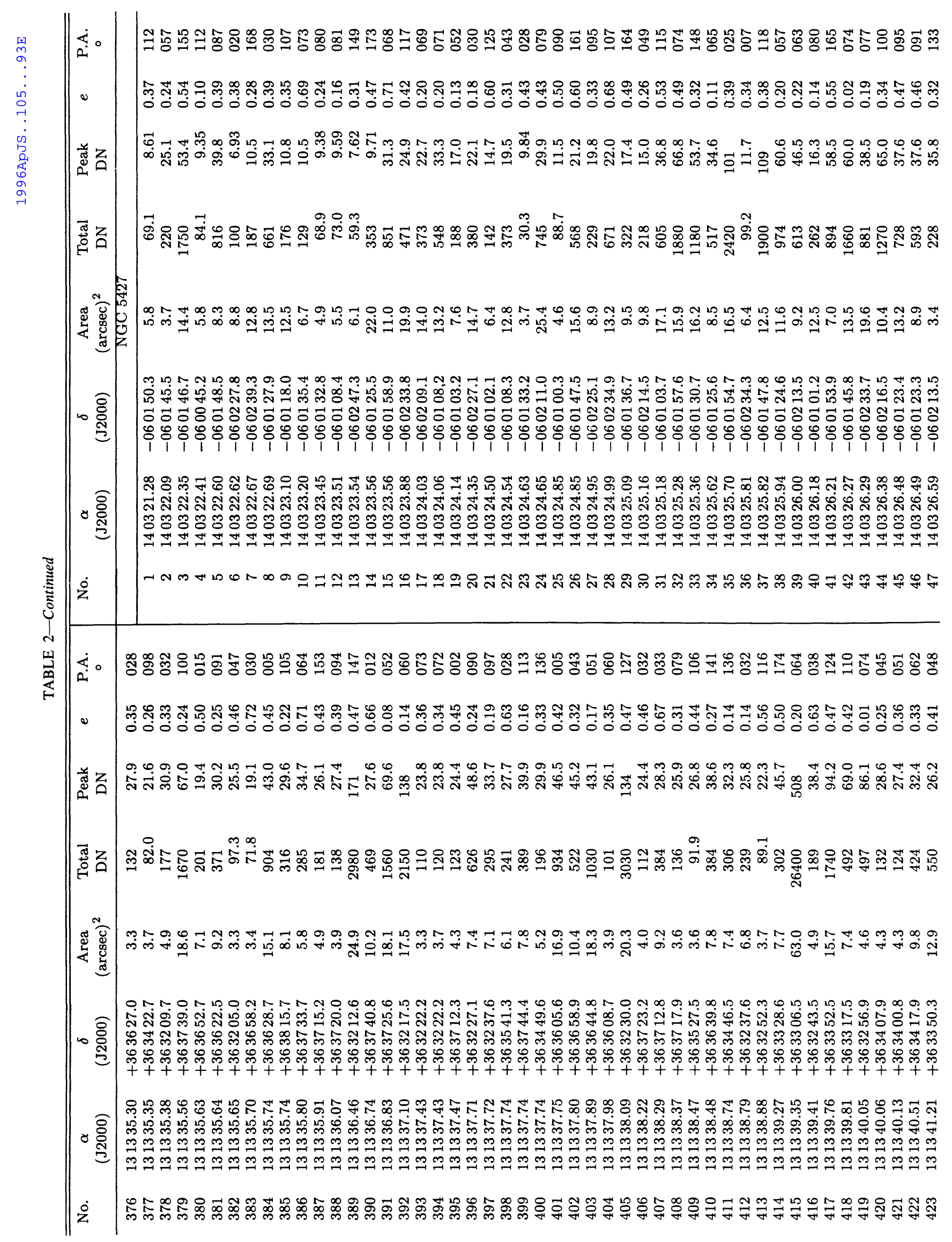




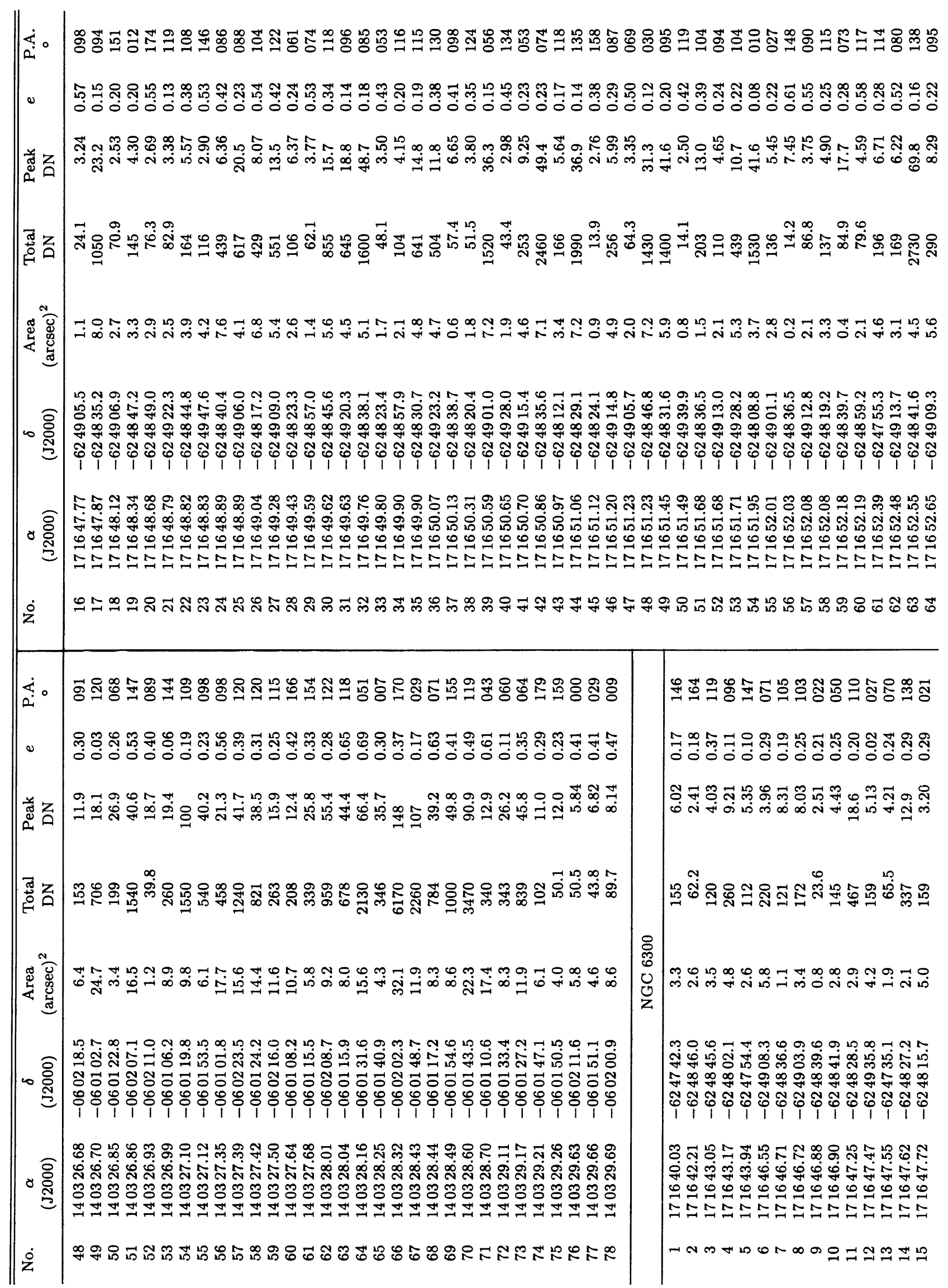




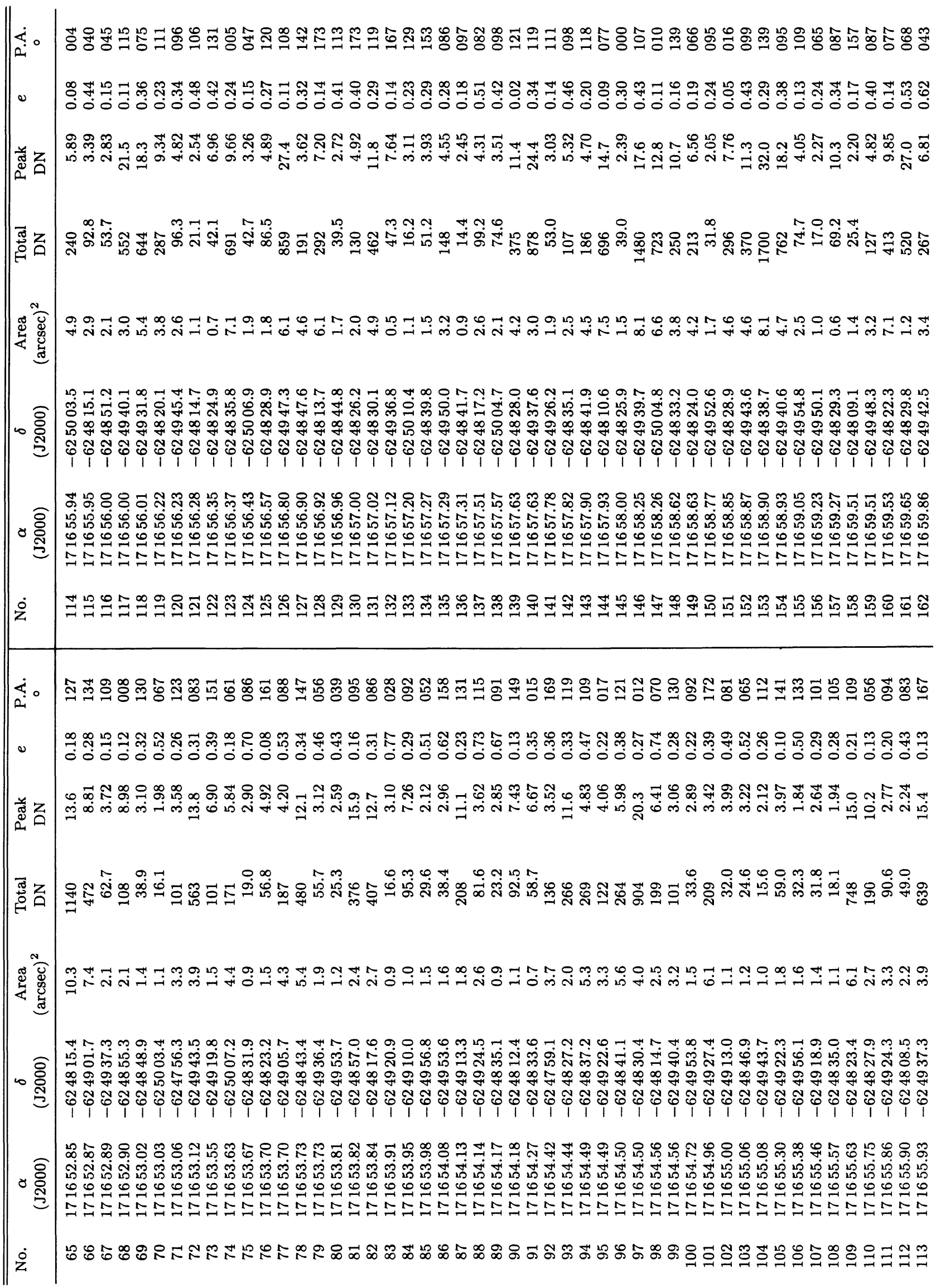




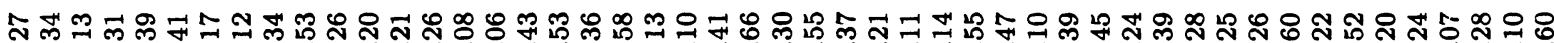

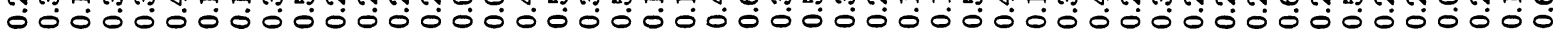

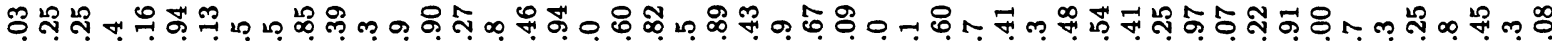

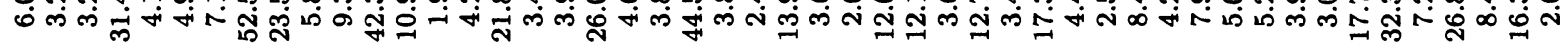

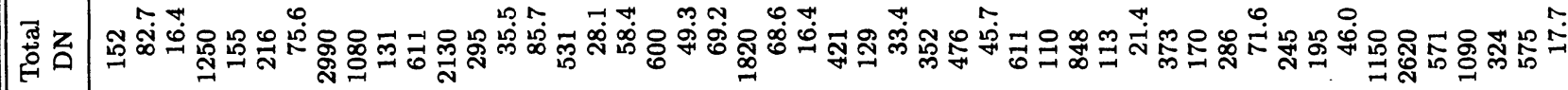

ช

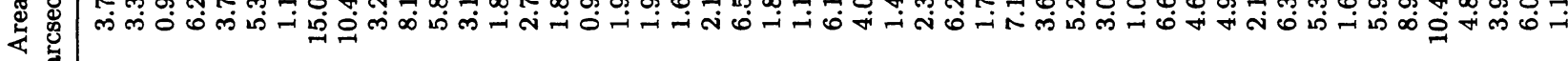

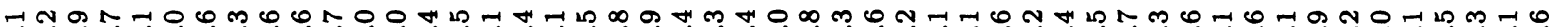
க் w 영

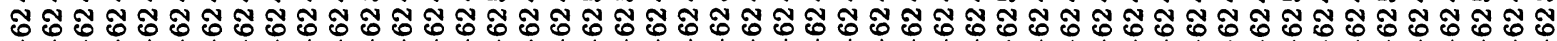

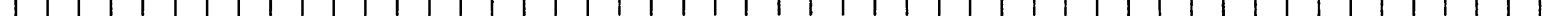

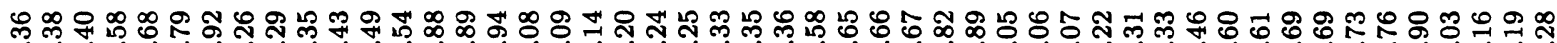

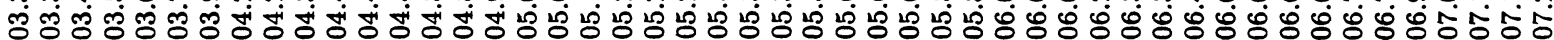

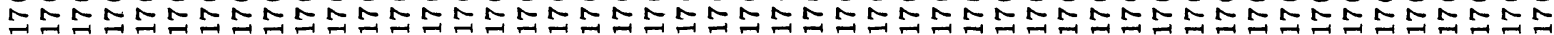

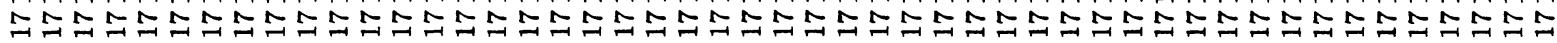

字 국

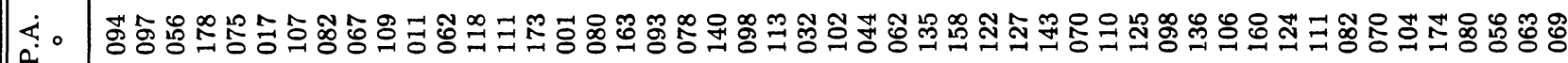

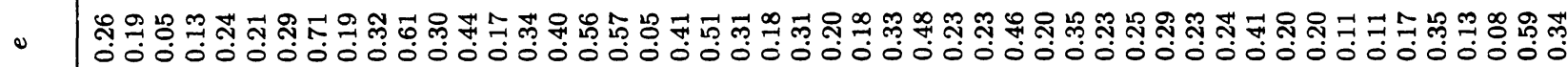

우

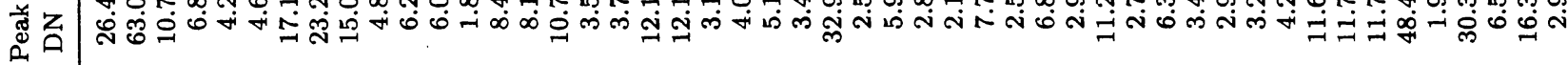

晋台 选兽

ᄀด八 ம்

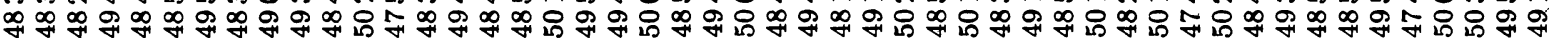

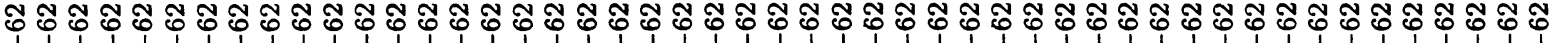

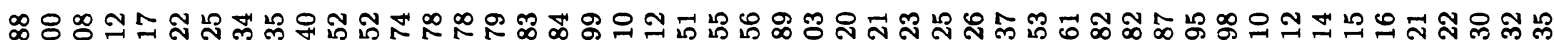

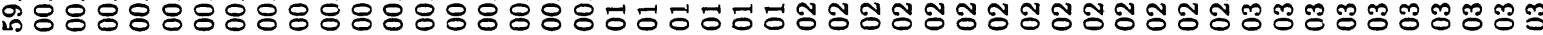

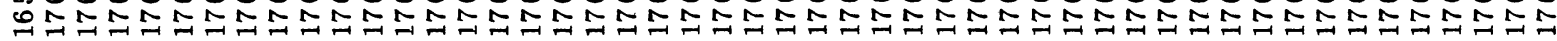

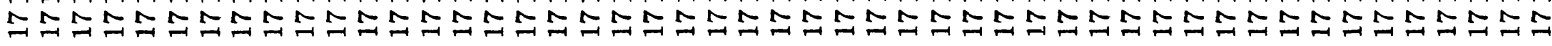
$\dot{0}$

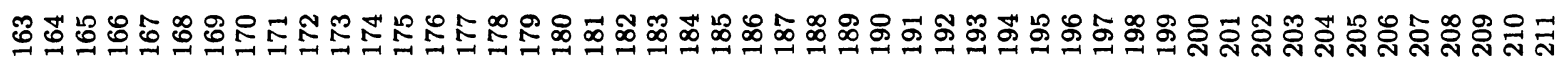




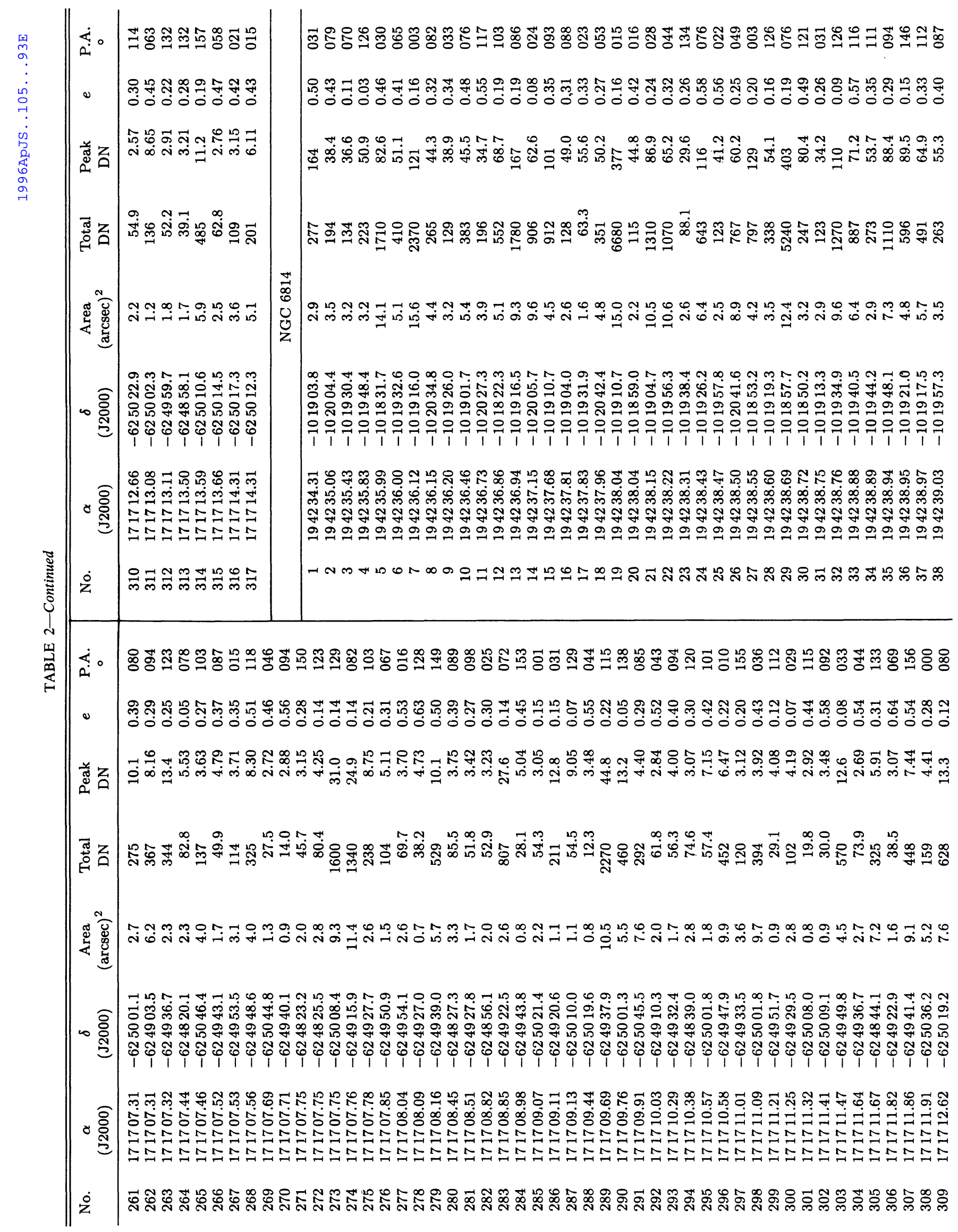




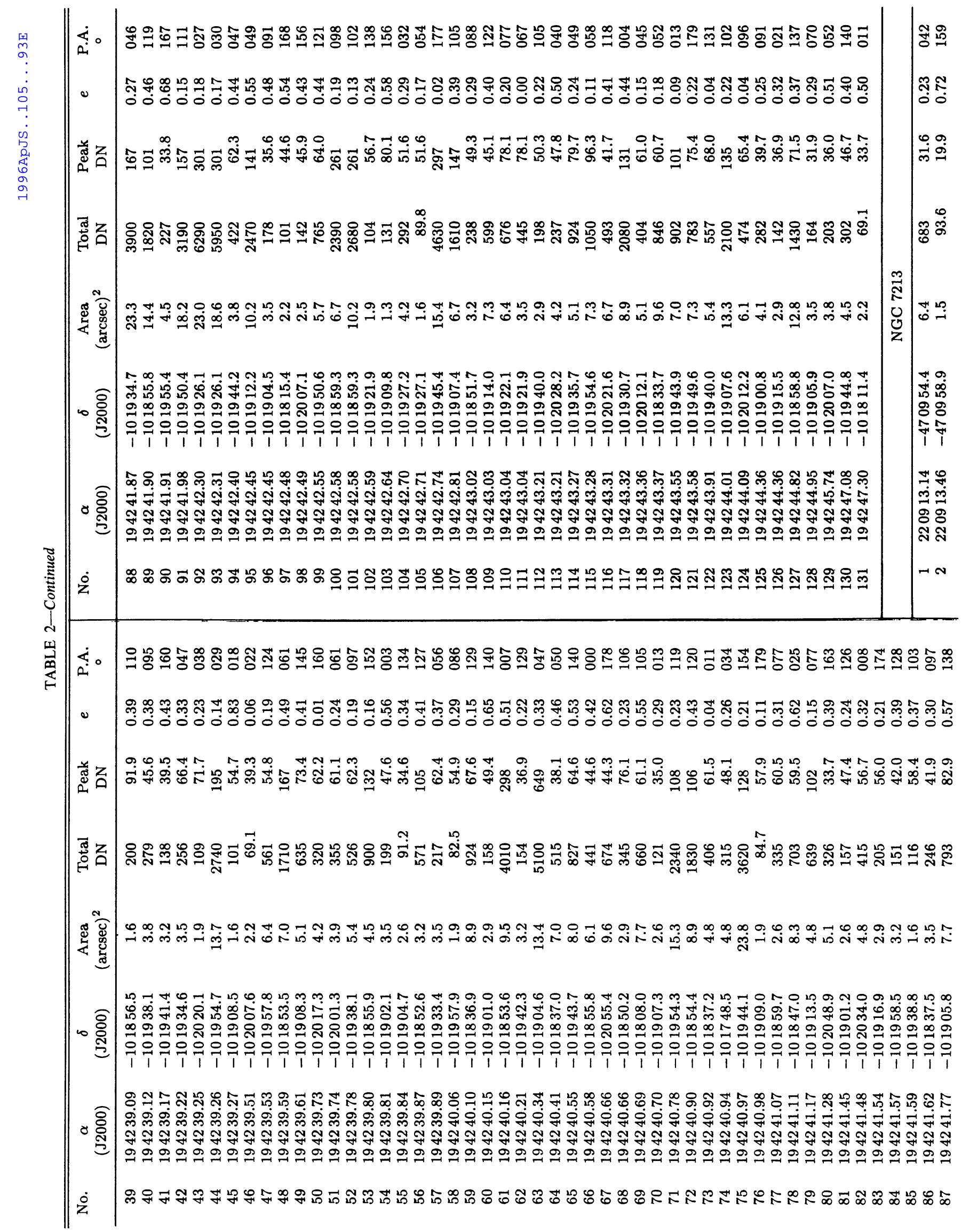

121 


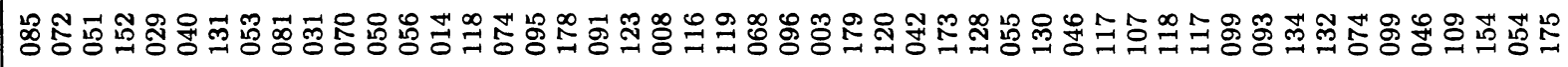

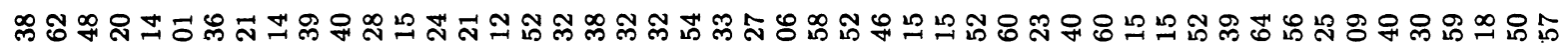
○் ó

节台

蛋Z

W0 HO-m N N N 递兽

O \#U H స் ไี่

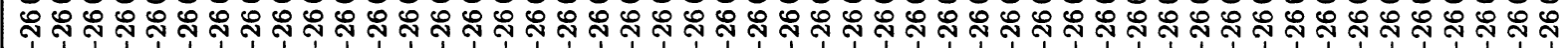

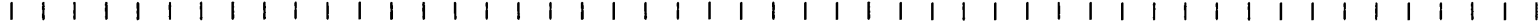

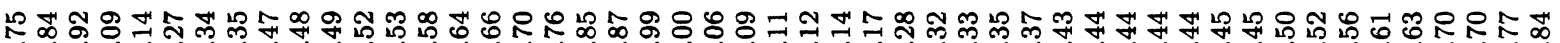

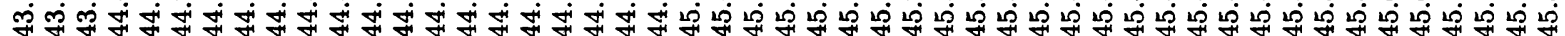
卢

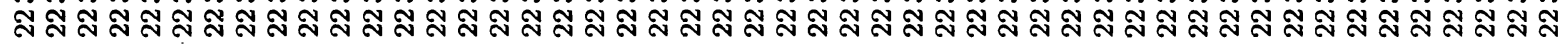

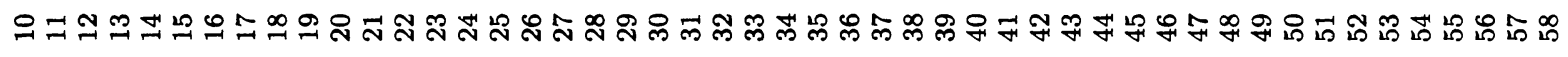

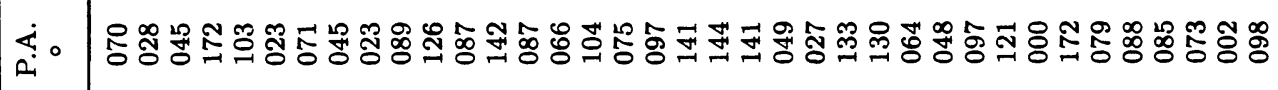
○

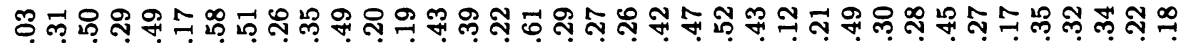

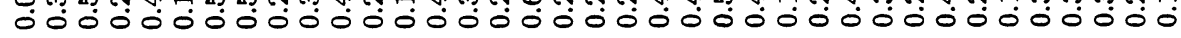

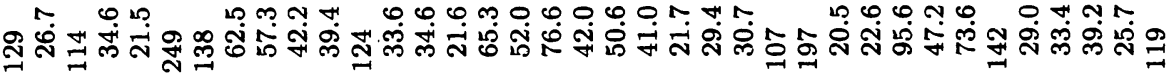

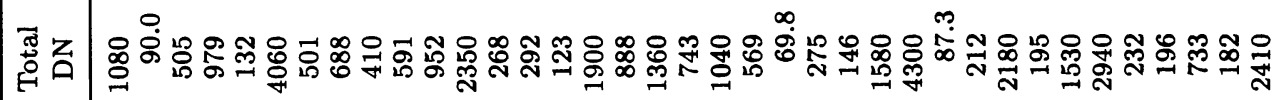

OmoON -

HמN

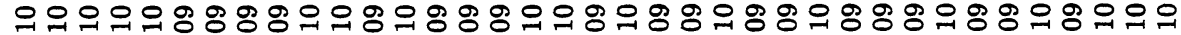

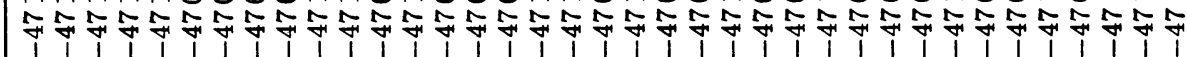

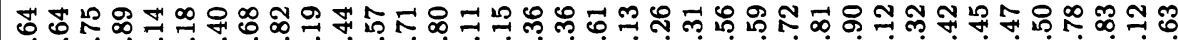

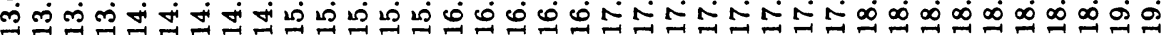

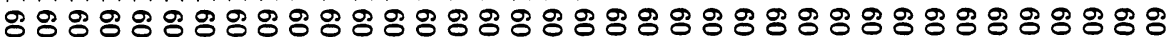
ส ๓

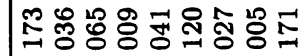

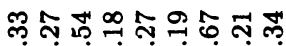

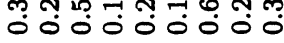

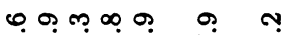

ஸ્ง

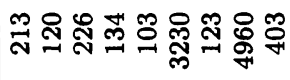

范

$m$ n $00-\infty \sim \infty 0$ 更

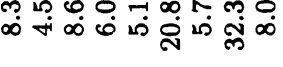

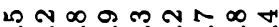
ฝิ่

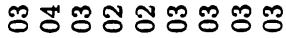

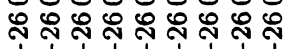
$1,1\} 1,1\}$

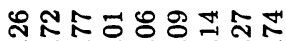

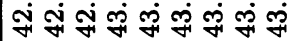

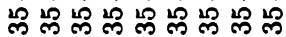
ส ส $~ \mathcal{~ N ~ ส ~ ส ~ ส ~}$

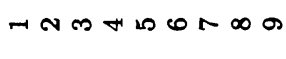




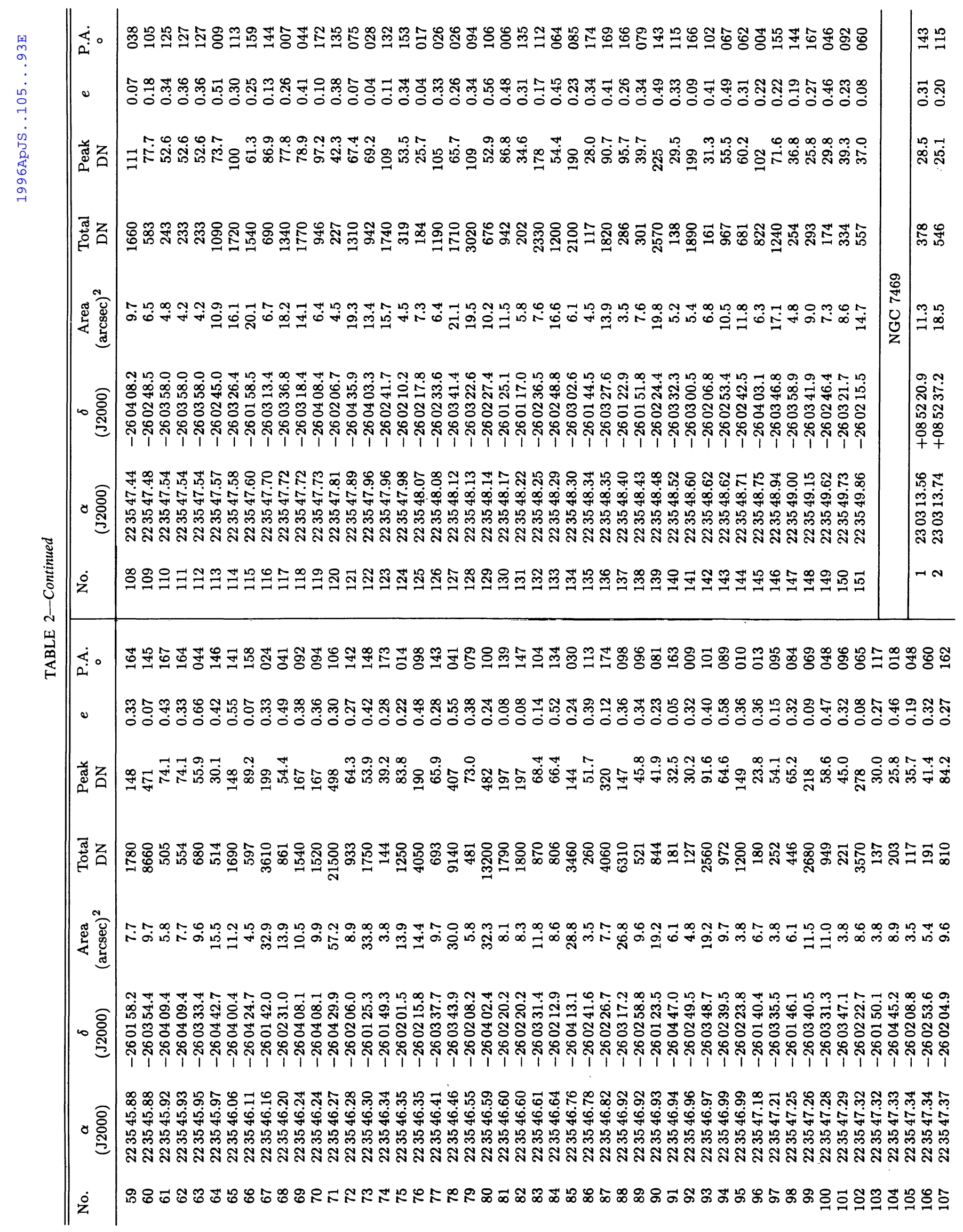


TABLE 2-Continued

\begin{tabular}{rccccccc}
\hline \hline No. & $\begin{array}{c}\alpha \\
(\mathrm{J} 2000)\end{array}$ & $\begin{array}{c}\delta \\
(\mathrm{J} 2000)\end{array}$ & $\begin{array}{c}\text { Area } \\
(\mathrm{arcsec})\end{array}$ & $\begin{array}{c}\text { Total } \\
\text { DN }\end{array}$ & $\begin{array}{c}\text { Peak } \\
\text { DN }\end{array}$ & $e$ & $\begin{array}{c}\text { P.A. } \\
\circ\end{array}$ \\
\hline 3 & 230314.73 & +085256.4 & 4.8 & 676 & 87.9 & 0.16 & 008 \\
4 & 230314.88 & +085207.9 & 7.1 & 125 & 20.9 & 0.19 & 067 \\
5 & 230314.91 & +085254.3 & 6.8 & 1200 & 134 & 0.19 & 044 \\
6 & 230315.03 & +085230.8 & 1.9 & 141 & 41.3 & 0.31 & 075 \\
7 & 230315.05 & +085227.7 & 1.3 & 62.4 & 36.3 & 0.59 & 020 \\
8 & 230315.10 & +085236.2 & 17.0 & 4380 & 196 & 0.30 & 027 \\
9 & 230315.25 & +085242.7 & 4.2 & 297 & 49.2 & 0.44 & 002 \\
10 & 230315.25 & +085242.7 & 6.1 & 360 & 49.2 & 0.42 & 007 \\
11 & 230315.48 & +085248.6 & 4.5 & 61.1 & 18.7 & 0.35 & 028 \\
12 & 230315.61 & +085218.7 & 3.5 & 796 & 114 & 0.41 & 010 \\
13 & 230316.01 & +085249.5 & 3.5 & 70.1 & 28.1 & 0.32 & 170 \\
14 & 230316.22 & +085221.2 & 7.0 & 1140 & 102 & 0.08 & 029 \\
15 & 230316.24 & +085224.2 & 1.9 & 150 & 43.9 & 0.25 & 138 \\
16 & 230316.25 & +085229.5 & 1.6 & 77.0 & 42.6 & 0.30 & 017 \\
17 & 230316.61 & +085249.2 & 15.7 & 1220 & 65.0 & 0.52 & 160 \\
18 & 230316.95 & +085216.6 & 5.5 & 91.2 & 22.9 & 0.11 & 094 \\
\hline
\end{tabular}

NoTE.- Table 2 is published in its entirety in computer-readable form in the AAS CD-ROM Series, Vol. 7.

should not exceed approximately $1 \%-2 \%$ for the faintest sources.

\section{NOTES ON INDIVIDUAL GALAXIES}

\section{1. $N G C 788$}

Since being identified as a Seyfert galaxy by Huchra, Wyatt, \& Davis (1982), NGC 788 has been observed in the optical (e.g., Hamuy \& Maza 1987; Wagner 1987; Kay 1994; Cruz-Gonzalez et al. 1994), radio (Ulvestad \& Wilson 1989), and millimeter (Heckman et al. 1989) wave bands. Morphologically, the galaxy is a lenticular/early-type spiral with faint spiral arms visible $\sim 30^{\prime \prime}$ radius from the nucleus. The spiral arms are brightest to the northwest of the nucleus, and Figure 1 reveals a string of bright, compact $\mathrm{H}$ II regions that trace the spiral arm, together with a complex of fainter $\mathbf{H}$ II regions associated with the southern arm.

\section{2. $N G C 1068$}

The prototypical Seyfert 2 galaxy, NGC 1068, has been investigated extensively. Spectropolarimetric observations (Antonucci \& Miller 1985) reveal broad Balmer-line emission associated with the nucleus, while optical spectrophotometry (Evans \& Dopita 1986) and narrow-band emission-line imaging (Pogge 1988) reveal the presence of high-excitation gas distributed in a conelike morphology to the northeast of the nucleus. A large system of bright H II regions is associated with the spiral arms, and a starburst ring surrounds the nucleus. From optical spectrophotometry of 13 giant extragalactic $H$ II regions located within the inner one-third of the disk, Evans \& Dopita (1987) derive a mean oxygen abundance that is slightly overabundant compared to the solar value and find no evidence for a radial abundance gradient. $H$ II region abundance measurements in the inner disk by Zaritsky et al. (1994) are consistent with zero radial gradient. The lack of a steep radial abundance gradient may be related to the gravitational potential produced by a stellar bar that is detected in the near-infrared (Scoville et al. 1988). Numerical simulations by Schwarz (1981) demonstrated that such a potential tends to produce strong radial flows into the nucleus on a timescale short compared to the age of the galaxy.
Figure 1 reveals a ring of bright, compact $\mathrm{H}$ II regions $\sim 10^{\prime \prime}-15^{\prime \prime}$ in radius from the nucleus. The ring is surrounded by an agglomeration of fainter, compact $\mathrm{H}$ II regions and diffuse $\mathrm{H} \alpha$ emission associated with the inner spiral arms within a radius of approximately $1^{\prime}$. Giant $\mathrm{H}$ II regions trace spiral arm structure out to a distance of order 3 ' from the nucleus.

\subsection{NGC 1097}

Although originally classified as a LINER on the basis of the optical emission-line spectrum (Keel 1983b), the recent appearance of broad Balmer-line emission and a featureless blue continuum implies that NGC 1097 contains a Seyfert 1 nucleus (Storchi-Bergmann, Baldwin, \& Wilson 1993). A nuclear ring of $\mathrm{H}$ II regions delineates a massive burst of star formation that may be related to the inner Lindblad resonance (e.g., Hummel, Van Der Hulst, \& Keel 1987; Storchi-Bergmann et al. 1995). Storchi-Bergmann, Calzetti, \& Kinney (1994) determine from the UV to near-IR spectral energy distribution of the starburst ring that the metal abundances in the gas are enhanced slightly relative to the solar values. Gaseous abundances for individual $\mathrm{H}$ II regions both in the ring and further out along the bar, as well as the gradient of oxygen abundance, are obtained by Storchi-Bergmann et al. (1995).

Numerous complexes of giant $\mathrm{H}$ II regions are associated with the bar beyond $\sim 50^{\prime \prime}$ from the nucleus. They are readily visible tracers of the structure of the spiral arms from where the arms connect to the ends of the bar to well beyond $3^{\prime}$ radius. Outside the nucleus starburst ring, the brightest and largest $\mathrm{H}$ II region complexes are located near the regions in which the bar and the inner spiral arms intersect. The star formation rate in these complexes may be enhanced through the interaction of radial gas flows along the bar with the rotational flows in the spiral structure.

\section{4. $N G C 1672$}

The nucleus of this barred spiral Seyfert 2 galaxy is surrounded by a starburst region containing several bright $\mathrm{H} \alpha$ emission-line knots within $\sim 10^{\prime \prime}$ radius. Within the starburst region, Storchi-Bergmann et al. (1994) measure an oxygen abundance that is approximately twice the solar 
value. Detailed observations of individual $\mathrm{H}$ II regions in the ring and further out, with abundances and gradient determinations, are presented by Storchi-Bergmann et al. (1995). Bright $H$ II region complexes trace the bar and inner and outer spiral arms. Additionally, diffuse $\mathrm{H} \alpha$ emission appears colocated with faint compact $\mathbf{H}$ II regions in the inner disk within $\sim 1^{\prime}$.

\section{5. $N G C 2782$}

NGC 2782 (Arp 215) is a well-studied, peculiar, earlytype spiral galaxy containing a nuclear starburst and several H II regions reported by Hodge \& Kennicutt (1983). Using spatially resolved spectrophotometry, Boer, Schulz, \& Keel (1992) identify a shell of highly ionized material $4^{\prime \prime}-8$ " S of the central starburst. They conclude that the local ISM is either shock heated or photoionized by warmers (Terlevich \& Melnick 1985). Smith (1994) has obtained high-quality broadband $B V R I$ images and presents a contour map of the brightest $\mathrm{H} \alpha$ emission.

Our data (Fig. 1) reveal diffuse $\mathrm{H} \alpha$ emission covering the entire region within $\sim 14^{\prime \prime}$ of the nucleus, in addition to the emission detected by Boer et al. A complex of bright but relatively diffuse $\mathrm{H}$ II regions is visible clearly $20^{\prime \prime}-25^{\prime \prime} \mathrm{N}$ of the nucleus, and a string of bright, compact $\mathbf{H}$ II regions is visible $\sim 30^{\prime \prime} \mathrm{W}$ of the nucleus. Fainter diffuse $\mathrm{H} \alpha$ emission is detected at larger radii.

\section{6. $N G C 3081$}

Phillips, Charles, \& Baldwin (1983) identified this earlytype ring galaxy as a Seyfert 2 on the basis of spectroscopic observations of narrow, high-ionization emission-lines detected from the nucleus. Cruz-Gonzalez et al. (1994) recently obtained additional optical spectrophotometry. Imaging reveals a bright nucleus with an apparent bulge component visible out to a radius of $\sim 10^{\prime \prime}$, and a superimposed inner barlike structure with P.A. $\sim 120^{\circ}$. A faint bar with P.A. $\sim 60^{\circ}$ leads to a ring structure with radius $\sim 25^{\prime \prime}-$ $30^{\prime \prime}$. The outer ring structure is similar to that observed in Hoag's object. Brosch (1985) suggests that in the latter object the ring was formed no more than a few Gyr ago as a result of an extreme bar instability. Possibly, NGC 3081 represents an earlier stage in the evolution of such an object, when the bar is still clearly visible. Figure 1 reveals numerous $\mathrm{H}$ II regions associated with the main stellar ring, and possibly faint, diffuse $\mathrm{H} \alpha$ emission associated with the inner bar/bulge component.

\subsection{NGC 4051}

This nucleus of this famous Seyfert 1 galaxy has been the subject of many intensive studies (e.g., de Robertis \& Osterbrock 1984; Pogge 1989; Veilleux 1991; Hunt et al. 1992; Kunieda et al. 1992). Detailed optical emission-line and continuum contour maps of the inner few arcseconds surrounding the nucleus are available (Haniff, Wilson, \& Ward 1988). Byrd, Sundelius, \& Valtonen (1987) suggest that the Seyfert activity may have been triggered tidally through interactions with NGC 4013, which is separated from NGC 4051 by $\sim 240 \mathrm{kpc}$.

Figure 1 demonstrates that the brightest giant $\mathrm{H}$ II regions trace the spiral arms structure out to $\sim 2^{\prime}$ radius. Compact $\mathrm{H}$ II regions are discernible individually closer to the nucleus, down to $\sim 15^{\prime \prime}$ radius. Within this radius, there is evidence for widespread, diffuse $\mathrm{H} \alpha$ emission rather than distinct compact $\mathrm{H} \alpha$ sources.

\section{8. $N G C 4593$}

The properties of the Seyfert 1 nucleus in this barred spiral have been investigated in detail. Optical and infrared photometry (e.g., Winkler et al. 1992; Kotilainen, Ward, \& Williger 1993; Kotilainen \& Ward 1994), and spectrophotometry (e.g., Kollatschny \& Fricke 1985; Crenshaw 1986; Morris \& Ward 1988) of the nucleus has been obtained at several epochs. These data show considerable variability of the optical nuclear continuum, which is observed also at ultraviolet (e.g., Clavel et al. 1983; Clavel 1983; Koratkar \& Gaskell 1991) and X-ray (Ghosh \& Soundarajaperumal 1993) wavelengths.

Giant extragalactic $H$ II regions tracing the spiral arm structure from an inner radius of $\sim 30^{\prime \prime}$ out to $\sim 80^{\prime \prime}$ are readily visible in Figure 1 . Most of the $H$ II regions are compact, with little evidence for the presence of diffuse $\mathrm{H} \alpha$ emission. Unlike most other barred spirals discussed in this paper, H II regions in NGC 4593 do not appear to be distributed along the bar. One possible explanation may be that the radial gas mixing along the bar that would ordinarily trigger star formation is relatively weak in this object.

\subsection{NGC 4639}

Pogge (1989) has obtained both narrow-band $\mathrm{H} \alpha+[\mathrm{N} \mathrm{II}] \lambda \lambda 6548,6583$ and [O III $] \lambda 5007 \mathrm{CCD}$ images of this object. Measurements of the total UV continuum flux from the galaxy at $1650 \AA$ and $2000 \AA$ are published by Deharveng et al. (1994) and Donas et al. (1987), respectively. Inspection of Figure 1 reveals numerous giant extragalactic $\mathrm{H}$ II regions visibly distributed along the spiral arms. The concentration and surface brightness of the $\mathrm{H}$ II regions appears to be largest in the spiral arms near the ends of the bar, although none are visible along the bar itself. Star formation may be enhanced in the regions of the arms near the ends of the bar through mechanical interactions in the local ISM.

\subsection{NGC 4939}

Numerous $H$ II regions delineate the spiral structure in this late-type galaxy. $\mathrm{H} \alpha$ images have been published previously by Hodge \& Kennicutt (1983). The stellar population and emission-line gas within $5 \mathrm{kpc}$ of the active nucleus is investigated by Storchi-Bergmann, Bica, \& Pastoriza $(1990 \mathrm{a}, \mathrm{b})$. They conclude based on photoionization models that the abundances are approximately solar $\left(Z_{\text {ISM }} \sim 1-2 Z_{\odot}\right)$ with the exception of nitrogen. The latter element appears to require a factor of $\sim 2.5$ overabundance with respect to oxygen for the models to match the observed emission-line strengths.

\subsection{NGC 5033}

The late-type X-ray-bright spiral is classified as a Seyfert 1 on the basis of observations of broad Balmer emission from the nucleus (Shuder 1980; Keel 1983c; Filippenko \& Sargent 1985). Large (3') aperture $\mathrm{H} \alpha+[\mathrm{N}$ II $] \lambda \lambda 6548,6583$ photometry is reported by Kennicutt \& Kent (1983), while near $(0.35-3.5 \mu \mathrm{m})$ infrared and IRAS photometry are presented by McAlary et al. (1983) and Rodriguez Espinoza, Rudy, \& Jones (1987), respectively. Optical emission-line imaging is reported by several authors (e.g., Keel 1983a; Elmegreen \& Elmegreen 1983; Pogge 1989a).

Zaritsky et al. (1994) use optical spectrophotometry to estimate the abundances of eight $\mathrm{H}$ II regions in the disk of NGC 5033, and they conclude that there is a strong abun- 
dance gradient, with an inferred nuclear (zero radius) oxygen abundance that is approximately twice solar. Our continuum-subtracted $\mathrm{H} \alpha$ image (Fig. 1) reveals the presence of bright $\mathbf{H}$ II regions in the inner spiral arms down to a distance of $\sim 5^{\prime \prime}$ from the nucleus. Giant extragalactic $\mathrm{H}$ II regions clearly trace the spiral arms out to at least $4^{\prime}$ radius, providing an excellent sample for abundance measurements.

\subsection{NGC 5427}

NGC 5427 is one component of the interacting pair of galaxies Arp 271. The other component is NGC 5426, which is separated from NGC 5427 by $\sim 25 \mathrm{kpc}$ (Dahari 1984). Optical spectra of the nucleus of NGC 5427 have been obtained by Kennicutt \& Keel (1984), who suggest that the nuclear activity may have been induced through the galaxy-galaxy interactions. Optical nuclear emissionline (Keel et al. 1985), infrared (Giuricin 1994), and radio (Giuricin et al. 1990) fluxes are available in the literature.

Figure 1 reveals the presence of a possible nuclear ring of $H$ II regions with a radius of $\sim 5^{\prime \prime}$. Numerous $H$ II region complexes trace the distorted spiral arms out to at least $1^{\prime}$ from the nucleus. The $\mathbf{H}$ II regions appear brightest in the eastern spiral arm, although there is a bright complex located in the western arm at the point at which the arm abruptly changes direction. One can speculate that mechanical interactions due to changes in the direction of the gas flows in the local ISM may be responsible for the star formation that has produced the $\mathrm{H}$ il region complex at this location.

\subsection{3. $N G C 6300$}

NGC 6300 was first classified as a Seyfert 2 galaxy on the basis of the nuclear emission-line profiles from optical spectrophotometry obtained by Phillips et al. (1983). Additional optical spectra were obtained by Bonatto, Bica, \& Alloin (1989), who conclude that shock heating may play a role in the ionization balance of the nucleus. Storchi-Bergmann \& Pastoriza (1989) obtained further optical spectrophotometry, and on the basis of comparison with photoionization models, they concluded that the nuclear $\mathrm{N} / \mathrm{O}$ ratio must be enhanced by a factor of $\sim 3$ above the solar value. An $\mathrm{H} \alpha$ image of the object is presented by Ryder \& Dopita (1993). Our continuum-subtracted $\mathrm{H} \alpha$ image (Fig. 1) reveals a wealth of compact $H$ II regions and diffuse $H \alpha$ emission, both in the ring structure and extending out to over $2^{\prime}$ from the nucleus. There is some evidence for weak $\mathrm{H} \alpha$ emission interior to the ring, although this is mostly diffuse. This is a low galactic latitude object and suffers from having numerous faint foreground stars in the field. As discussed in $\S 3$, a small fraction of these may be misclassified as emission-line sources.

\subsection{4. $N G C 6814$}

The nuclear variability of NGC 6814 has been investigated extensively. Optical photometry and spectrophotometry (e.g., Morris \& Ward 1988; Sekiguchi \& Menzies 1990; Winkler et al. 1992; Winkler 1992) reveal that the broad nuclear $\mathrm{H} \beta$ flux can vary by factors of $2-4$ over a period of a few months to a few years, with smaller variations and continuum variability occurring on shorter timescales. Figure 1 reveals numerous giant extragalactic $\mathrm{H}$ II regions tracing both the inner and outer spiral arms. The inner spiral arms are particularly congested. An $\mathrm{H} \alpha$ image of the galaxy was obtained by Knapen et al. (1993), who studied the statistical properties of the $\mathrm{H}$ II region population from measurements of over $700 \mathrm{H}$ II regions. Additional $\mathrm{H} \alpha+[\mathrm{N}$ II $] \lambda \lambda 6548,6583$ and [O III $\lambda 5007$ images have been published by Pogge (1989a). Like NGC 6300, this is a low galactic latitude object with some field contamination from foreground stars.

\subsection{5. $N G C 7213$}

This early-type spiral has been classified variously as a LINER because of the presence of strong low-ionization nuclear emission lines such as [O I] $\lambda \lambda 6300,6364$ (e.g., Phillips et al. 1986), and as a Seyfert 1 because of the presence of broad Balmer emission. Our continuum-subtracted $\mathrm{H} \alpha$ image reveals the presence of bright, compact $\mathrm{H}$ il regions arranged in a ringlike morphology located $\sim 20^{\prime \prime}-40^{\prime \prime}$ in radius from the nucleus.

\subsection{NGC 7314}

Inspection of Figure 1 reveals numerous giant $\mathrm{H}$ II regions distributed throughout the arms of this highly inclined late-type spiral. Within the arms, bright, compact $\mathrm{H}$ II regons typically appear to be embedded in a diffuse $\mathrm{H} \alpha$ background that may trace the spiral structure. The region within $\sim 10^{\prime \prime}$ of the nucleus appears to be relatively free of $\mathrm{H} \alpha$ emission. Spectrophotometry of the nucleus has been published by several investigators (e.g, Morris \& Ward 1985, 1988; Schulz, Knake, \& Schmidt-Kaler 1994), with the former authors detecting the $\mathrm{O}_{\mathrm{I}} \lambda 8446$ Bowen fluorescence line, suggesting that a high-density, optically thick component may be present in the nucleus. Optical and near-infrared photometry of the nucleus and surrounding regions has been obtained by Kotilainen and coworkers (e.g., Kotilainen et al. 1993; Kotilainen \& Ward 1994).

\subsection{7. $N G C 7469$}

This early-type Seyfert 1 galaxy has been investigated in detail from X-ray (Brandt et al. 1993) and UV (Westin 1984) wave bands through to medium-IR wavelengths (e.g., Rudy et al. 1982; Keto et al. 1992; Miles, Houck, \& Hayward 1994). Long-slit spectra and CCD imaging of the nuclear and immediately surrounding regions (de Robertis \& Pogge 1986) show evidence for ongoing star formation in the circumnuclear ring. This may be driven by cloud-cloud collisions due to the outflow of material from the nucleus (Westin 1985). Additional optical emission-line and continuum images, and radio continuum data, of the nuclear regions are published by Wilson et al. (1991), and Mauder et al. (1994) present speckle masking images of the inner nuclear region.

Additional long-slit spectra have been obtained by Wilson et al. (1986) and Bonatto \& Pastoriza (1990). The latter authors conclude that the elemental abundances at $\sim 8^{\prime \prime}$ from the nucleus are approximately solar, with the exception of nitrogen, which appears to be enhanced by a factor of 2 over the solar value. Compact $\mathrm{H}$ II regions overlaying a diffuse $H \alpha$ background are clearly visible within $\sim 10^{\prime \prime}$ of the nucleus in Figure 1. Further out, a few giant extragalactic $\mathrm{H}$ II regions are evident and appear to trace the weak spiral arm structure out to a distance of order $50^{\prime \prime}$ from the nucleus.

The authors would like to thank Jack Baldwin for obtaining the images of several of the objects included in 
this atlas and for helping with the data reduction for these objects. Sharon Pesto performed the initial astrometric calibrations for some of the images. Dr. Brian McLean is the author of the XSPAM package used for the astrometric calibrations, and his advice during this phase of the work is greatly appreciated.

\section{REFERENCES}

Antonucci, R. R. J., \& Miller, J. S. 1985, ApJ, 297, 621

Boer, B., Schulz, H., \& Keel, W. C. 1992, A\&A, 260, 67

Bonatto, C., Bica, E., \& Alloin, D. 1989, A\&A, 226, 23

Bonatto, C. J., \& Pastoriza, M. G. 1990, ApJ, 353, 455

Brandt, W. N., Fabian, A. C., Nandra, K., \& Tsuruta, S. 1993, MNRAS, 265,996

Brosch, N. 1985, A\&A, 153, 199

Byrd, G. G., Sundelius, B., \& Valtonen, M. 1987, A\&A, 171, 16

Clavel, J. 1983, MNRAS, 204, 189

Clavel, J., Joly, M., Collin-Souffrin, S., Bergeron, J., \& Penston, M. 1983, MNRAS, 202, 85

Crenshaw, D. M. 1985, ApJS, 62, 821

Cruz-Gonzalez, I., Carrasco, L., Serrano, A., Guichard, J., Dultzin-Hacyan, D., \& Bisiachhi, G. F. 1994, ApJS, 94, 47

Dahari, O. 1985, AJ, 90, 1772

Deharveng, J.-M., Sasseen, T. P., Buat, V., Bowyer, S., Lampton, M., \&

$\mathrm{Wu}, \mathrm{X} .1994, \mathrm{~A} \& \mathrm{~A}, 289,715$

de Robertis, M. M., \& Pogge, R. W. 1986, AJ, 91, 1026

de Robertis, M. M. \& Osterbrock, D. E. 1984, ApJ, 286, 171

de Vaucouleurs, G., de Vaucouleurs, A., Corwin, H. G., Jr., Buta, R. J., Paturel, G., \& Fouqué, P. 1991, Third Reference Catalogue of Bright Galaxies (New York: Springer)

Dinerstein, H. L. 1990, in The Interstellar Medium in Galaxies, ed. H. A. Thronson \& J. M. Shull (Dordrecht: Kluwer), 257

Donas, J., Deharveng, J.-M., Laget, M., Milliard, B., \& Huguenin, D. 1987, A\&A, 180, 12

Dopita, M. A., \& Evans, I. N. 1986, ApJ, 307, 431

Edmunds, M. G., \& Pagel, B. E. J. 1984, MNRAS, 211, 507

Elmegreen, B. G., \& Elmegreen, D. M. 1983, MNRAS, 203, 31

Evans, I. N. 1986, ApJ, 309, 544

Evans, I. N., \& Dopita, M. A. 1986, ApJ, 310, L15 .1987, ApJ, 319, 662

Filippenko, A. V., \& Sargent, W. L. W. 1985, ApJS, 57, 503

Ghosh, K. K., \& Soundararajaperumal, S. 1993, A\&A, 273, 397

Giuricin, G., Mardirossian, F., Mezzetti, M., \& Bertotti, G. 1990, ApJS, 72, 551

Giuricin, G., Tamburini, L., Mardirossian, F., Mezzetti, M., \& Monaco, P. 1994, ApJ, 427, 202

Hamuy, M., \& Maza, J. 1987, A\&AS, 68, 383

Haniff, C. A. Wilson, A. S. \& Ward, M. J. 1988, ApJ, 334, 104

Hawley, S. A., \& Phillips, M. M. 1980, ApJ, 235, 783

Heckman, T. M., Blitz, L., Wilson, A. S., Armus, L., \& Miley, G. K. 1989, ApJ, 342,735

Hodge, P. W. \& Kennicutt, R. C. 1983, AJ, 88, 296

Huchra, J. P., Wyatt, W. F., \& Davis, H. 1982, AJ, 87, 1628

Hummel, E., Van Der Hulst, J. M., \& Keel, W.C. 1987, A\&A, 172, 32

Hunt, L. K., Mannucci, F., Salvati, M., \& Stanga, R. M. 1992, A\&A, 257, 434

Kay, L. E. 1994, ApJ, 430, 196

Keel, W. C. 1983a, ApJ, 268, 632

$1983 \mathrm{~b}$, ApJ, 269, 466

$1983 \mathrm{c}$, ApJS, 52, 229

Keel, W. C., Kennicutt, R. C., Hummel, E., \& Van Der Hulst, J. M. 1985, AJ, 90,708

Kennicutt, R. C., \& Keel, W. C. 1984, ApJ, 279, L5

Kennicutt, R. C., \& Kent, S. M. 1983, AJ, 88, 1094

Keto, E., Ball, R., Arens, J., Jernigan, G., \& Meixner, M. 1992, ApJ, 389, 223

Knapen, J. H., Arnth-Jensen, N., Cepa, J., \& Beckman, J. E. 1993, AJ, 106, 56

Kollatschny, W., \& Fricke, K. J. 1985, A\&A, 143, 393

Koratkar, A. P., \& Gaskell, C. M. 1991, ApJS, 75, 719

Kotilainen, J. K., \& Ward, M. J. 1994, MNRAS, 266, 953

Kotilainen, J. K., Ward, M. J., \& Williger, G. M. 1993, MNRAS, 263, 655
Kunieda, H., Hayakawa, S., Tawara, Y., Koyama, K., Tsuruta, S., \& Leighly, K. 1992, ApJ, 384, 482

Lacey, C. G., \& Fall, S. M. 1985, ApJ, 290, 154

Lutz, R. K. 1979, Comput. J., 23, 262

MacGillivray, H. T., \& Stobie, R. S. 1984, Vistas Astron., 27, 433

Mauder, W., Weigelt, G., Appenzeller, I., \& Wagner, S. J. 1994, A\&A, 285, 44

Mayor, M \& Vigroux, L. 1981, A\&A, 98, 1

McAlary, C. W., McLaren, R. A., McGonegal, R. J., \& Maza, J. 1983, ApJS, 52, 341

Miles, J. W., Houck, J. R., \& Hayward, T. L. 1994, ApJ, 425, L37

Morris, S. L., \& Ward, M. J. 1985, MNRAS, 215, 57P . 1988, MNRAS, 230, 639

Pagel, B. E. J., Edmunds, M. G., Blackwell, D. E., Chun, M. S., \& Smith, G. 1979, MNRAS, 189, 95

Pagel, B. E. J., Edmunds, M. G., \& Smith, G. 1980, MNRAS, 193, 219

Phillips, M. M., Charles, P. A., \& Baldwin, J. A. 1983, ApJ, 266, 485

Phillips, M. M., Jenkins, C. R., Dopita, M. A., Sadler, E. M., \& Binette, L. 1986, ApJS, 91, 1062

Pogge, R. W. 1988, ApJ, 328, 519

. 1989, ApJ, 345, 730

Rodriguez Espinoza, J. M., Rudy, R. J. \& Jones, B. 1987, ApJ, 312, 555

Roy, J.-R., \& Walsh, J. R. 1987, MNRÄS, 228, 883

1988, MNRAS, 234, 977

Rudy, R. J., Jones, B. E., Le Van, P. D., Puetter, R. C., Smith, H. E., Willner, S. D., \& Tokunaga, A. T. 1982, ApJ, 257, 570

Russell, J. L., Lasker, B. M., McLean, B. J., Sturch, C. R., \& Jenkner, H. 1990, AJ, 99, 2059

Ryder, S. D., \& Dopita, M. A. 1993, ApJS, 88, 415

Schulz, H., Knake, A., \& Schmidt-Kaler, Th. 1994, A\&A, 288, 425

Schwarz, M. P. 1981, ApJ, 247, 77

Scoville, N. Z., Matthews, K., Carico, D. P., \& Sanders, D. B. 1988, ApJ, 327,61

Sekiguchi, K., \& Menzies, J. W. 1990, MNRAS, 245, 66

Shields, G. A., \& Searle, L. 1978, ApJ, 222, 821

Shuder, J. M. 1980, ApJ, 240, 32

Smith, B. J. 1994, AJ, 107, 1695

Storchi-Bergmann, T., Ardila, A., \& Wilson, A. S. 1996, in preparation

Storchi-Bergmann, T., Baldwin, J. A., \& Wilson, A. S. 1993, ApJ, 410, L11

Storchi-Bergmann, T., Bica, E., \& Pastoriza, M. G. 1990a, Rev. Mexicana Astron. Astrofis, 21, 23

. 1990b, MNRAS, 245, 749

Storchi-Bergmann, T., Calzetti, D., \& Kinney, A. L. 1994, ApJ, 429, 572

Storchi-Bergmann, T., \& Pastoriza, M. G. 1989, ApJ, 347, 195 .1990, PASP, 102, 1359

Storchi-Bergmann, T., Wilson, A. S., \& Baldwin, J. A. 1996, ApJ, 460, 252

Terlevich, R., \& Melnick, J. 1985, MNRAS, 213, 841

Tinsley, B. M., \& Larson, R. 1978, ApJ, 221, 446

Ulvestad, J. S., \& Wilson, A. S. 1989, ApJ, 343, 659

Veilleux, S. 1991, ApJ, 369, 331

Véron-Cetty, M.-P., \& Véron, P. 1993, A Catalogue of Quasars and Active Nuclei (6th ed.; Garching: ESO)

Wagner, S. J. 1987, PASP, 100, 54

Walsh, J. R., \& Roy, J.-R. 1989, ApJ, 341, 722

Westin, B. A. M. 1984, A\&A, 132,136 . 1985, A\&A, 151, 137

Wilson, A. S., Baldwin, J. A., Sze-Dung Sun, \& Wright, A. E. 1986, ApJ, 310,121

Wilson, A. S., Heifer, T. T., Haniff, C. A., \& Ward, M. J. 1991, ApJ, 381, 79

Winkler, H. 1992, MNRAS, 257, 677

Winkler, H., Glass, I. S., van Wyk, F., Marang, F., Spencer Jones, J. H., Buckley, D. A. H., \& Sekiguchi, K. 1992, MNRAS, 257, 659

Zaritsky, D., Kennicutt, R. C., Jr., \& Huchra, J. P. 1994, ApJ, 420, 87 